\title{
Geometric Analysis of the Doppler Frequency for General Non-Stationary 3D Mobile-to-Mobile Channels based on Prolate Spheroidal Coordinates
}

\author{
Michael Walter, Senior Member, IEEE, Dmitriy Shutin, Senior Member, IEEE, Martin Schmidhammer, \\ Member, IEEE, David W. Matolak, Senior Member, IEEE, Alenka Zajic, Senior Member, IEEE
}

\begin{abstract}
Mobile-to-mobile channels often exhibit timevariant Doppler frequency shifts due to the movement of transmitter and receiver. An accurate description of the Doppler frequency turns out to be very difficult in Cartesian coordinates, and any subsequent algebraic analysis of the Doppler frequency is intractable. In contrast to other approaches, we base our investigation on a geometric description of the Doppler frequency with the following three mathematical pillars: prolate spheroidal coordinate system, algebraic curve theory, and differential forms. The prolate spheroidal coordinate system is more appropriate to algebraically investigate the problem. After the transformation into the new coordinate system, the theory of algebraic curves is needed to resolve the ambiguities. Finally, the differential forms are required to derive the joint delay Doppler probability density function. This function is normalized by the equivalent ellipsoidal area of the scattering plane bounded by the delay ellipsoid. The results generalize in a natural way our previous model to a complete 3D description. Our solutions enable insight into the geometry of the Doppler frequency and we were able to derive a Doppler frequency that is dependent on the delay and the scattering plane. The presented theory allows describing any time-variant, single-bounce, mobile-to-mobile scattering channel.
\end{abstract}

Index Terms-Doppler frequency, mobile-to-mobile communication, geometry-based stochastic channel model, algebraic curve, prolate spheroidal coordinate system, differential forms.

\section{INTRODUCTION}

$\mathbf{M}$ OBILE-TO-MOBILE communication becomes more important by the day. Devices should be able to communicate with each other anywhere and anytime as the internet of things becomes more prevalent. Especially important are mobile devices which are not fixed at a certain location. The non-stationarity of the communication channel between mobile devices makes understanding of such wireless channels both challenging and interesting for research. A fundamental difference exists, however, in modeling the Doppler frequency for fixed-to-mobile or mobile-to-mobile channels.

In the past, when narrowband systems were deployed, purely stochastic models were often used to characterize the propagation channel [1]. Those stochastic models are based

M. Walter, D. Shutin, and M. Schmidhammer are with the German Aerospace Center (DLR), Institute of Communications and Navigation, 82234 Wessling, Germany (e-mail: \{m.walter, dmitriy.shutin, martin.schmidhammer\}@dlr.de), D. W. Matolak is with the College of Engineering and Computing, University of South Carolina, Columbia, SC 29208, USA (e-mail: matolak@cec.sc.edu), A. Zajic is with the School of Electrical and Computer Engineering, Georgia Institute of Technology, Atlanta, GA 30332, USA (e-mail: alenka.zajic@ece.gatech.edu). on the wide-sense stationary uncorrelated scattering (WSSUS) assumption that was introduced in the seminal paper of Bello [2]. The correlation function, which describes the power spectral density of delay and Doppler, is called scattering function. Power spectal densities can usually be recorded by measurement equipment. The joint delay Doppler probability density function (pdf) in this paper is based on the proof of proportionality between pdf and scattering function in [3]. The Bello model is valid for narrowband channels, so all diffuse components are essentially combined in the same tap due to the limited bandwidth. This WSSUS assumption is justified for narrowband fixed-to-mobile channels for some limited spatial extent or temporal duration.

For scatterers uniformly distributed in azimuth, Clarke derived in [1] the so called Jakes Doppler power spectrum [4]. A WSSUS model for the mobile-to-mobile (M2M) channel was introduced in [5]. M2M model extensions were derived in [6][9] for various $2 \mathrm{D}$ and $3 \mathrm{D}$ scenarios as well as for multiple input multiple output (MIMO) scenarios in [10]-[14].

For M2M channels, however, the WSSUS assumption is mostly violated due to the simultaneous movement of transmitter and receiver. This has been observed for example in vehicle-to-vehicle (V2V) channels according to [15] and [16]. This observation is also true for other M2M channels, e.g., the air-to-air (A2A) channel in [17].

To address statistical non-stationarity, Matz extended Bello's model in [18] to include a non-WSSUS description. The resulting model is represented by four-dimensional channel correlation functions, which are difficult to handle in practice. Following [19], which suggested to model the Doppler spectrum as a time-variant function, we proposed in [20] a simpler solution by considering time-variant, delay-dependent Doppler pdfs for V2V channels. Later in [21] we showed that the timevariant scattering function remains proportional to the timevariant joint delay Doppler pdf. The result thus relates the local scattering function of Matz with the time-variant joint delay Doppler pdf. Such a time-variant description has nowadays become more common in the mobile radio communication literature, see e.g. [22]. In [23] the proposed model was then extended to A2A scenarios. The theoretical models were verified using measurement data for both $\mathrm{V} 2 \mathrm{~V}$ channels in [24] and [25] and A2A channels in [26] and [27]; these results retained however the uncorrelated scattering (US) assumption. A validation of our model with $\mathrm{V} 2 \mathrm{~V}$ measurement data from TU Ilmenau was shown in [28]. 
Our proposed model enabled us to describe the time-variant channel accurately, but the underlying Cartesian coordinates have prevented both a thorough mathematical analysis of the Doppler frequency and an efficient implementation to simulate the channel. Because both transmitter (TX) and receiver (RX) are in the foci of the delay ellipsoid, a delay-dependent description better expresses the so called two-center problem. A fixed delay defines an ellipse for a 2D scenario and an ellipsoid for a 3D scenario, since this particular geometric shape defines the locations where the delay between TX and RX via a scatterer is constant. Typically, such two-center problems are advantageously represented in prolate spheroidal coordinates (PSCs). Thus, in [29], we transformed the timevariant, delay-dependent Doppler pdf description for $\mathrm{V} 2 \mathrm{~V}$ channels, which are a symmetric version of the general M2M channel, into PSCs. We derived the simpler delay-dependent Doppler pdf by assuming that the joint pdf can be factorized according to Bayes rule. Based on the transformed description, we obtained analytic expressions of the Doppler pdfs for timevariant V2V scenarios in [21].

Since the limiting frequencies of the Doppler frequency are important parameters for future M2M communication systems, we conducted an algebraic analysis to calculate the delaydependent limiting Doppler frequencies of the pdfs in [30]. Thereby, the derivative of the Doppler frequency represented in PSCs was converted into a polynomial to conduct the analysis. These results have been extended to A2A channels by exploiting the symmetry of the scattering plane in [17], i.e., the scattering plane is halved by the $y$-z-plane. The results are thus only valid for certain scattering planes in an M2M scenario and the extension is not yet applicable to the general case. The algebraic curves and the sixth order polynomial have less parameters due to the exploited symmetry.

In this paper, we present the mathematical methods to calculate both the delay-dependent Doppler pdf and the joint delay Doppler pdf for general M2M channels. By applying the algebraic curve theory we derive a closed-form equation of the delay-dependent Doppler frequency for an arbitrarily oriented scattering plane. This particularly parameterized Doppler frequency allows a bijective mapping from spatial coordinates to the delay Doppler coordinates. Thus, we are able to transform a spatial distribution of scatterers to both the delay-dependent Doppler pdf and the joint delay Doppler pdf. Motivated by [31] and [32], where differential forms are already successfully applied to electromagnetic field theory, we use differential forms to derive analytical expressions for the joint delay Doppler pdf based on the physically motivated bistatic path loss. Thus, the scattering power is accounted for in the joint delay Doppler pdf for the first time. The application of differential forms [33] is necessary to derive the differential areas and to carry out the two-dimensional integration in the prolate spheroidal coordinate system (PSCS). Eventually, our generalized M2M channel model is capable of describing any time-variant, single-bounce $\mathrm{M} 2 \mathrm{M}$ scenario with arbitrarily oriented scattering planes, e.g., V2V, A2A, drone-to-drone, or drone-to-vehicle scenarios. Numerical results show the validity of the presented equations for an exemplary drone-to-drone scenario.
The remainder of the paper is structured as follows. In Section II, we describe the general approach to derive the delaydependent and the joint delay Doppler probability density function and introduce the three main mathematical concepts. In Section III, we derive delay-dependent Doppler functions, i.e., the delay-dependent Doppler probability density function and the limiting frequencies of the probability density function of the mobile-to-mobile channel. Similar to the delaydependent Doppler probability density function, the joint delay Doppler probability function is derived in Section IV. The application of the presented mathematical derivations with regard to channel modeling and channel simulation are shown in Section V. The paper concludes with Section VI.

\section{The Three Theoretical Pillars}

We introduce the three main mathematical concepts and the general modeling approach that will be used to geometrically investigate the Doppler frequency. We motivate why these concepts are needed and explain the relationship between them. Then, we apply the presented concepts to compute both the delay-dependent Doppler pdf and the joint delay Doppler pdf.

\section{A. Doppler Frequency and Probability Density Functions in Prolate Spheroidal Coordinates}

It is known that for two-center problems PSCs are a more suitable representation than classical Cartesian coordinates. Moreover, the PSCS allows that the description of the Doppler frequency becomes free of trigonometric functions for arbitrarily oriented scattering planes, which simplifies a subsequent algebraic analysis. To derive the PSC description we first consider TX and RX moving with respect to a fixed east, north, up (ENU) Cartesian coordinate system $(\mathrm{CCS})(e, n, u)$ as shown in Fig. 1, where the $e-n$-plane is selected to coincide with the ground plane. Assuming typical communication distances for M2M links and typical scattering power decay rates, a flat scattering plane assumption is well justified in practice. Thus, the origin of the ENU coordinate system should be placed in the vicinity of TX and RX. If both move too far away, the location of the ENU coordinate system should be changed. For modeling the channel between TX and RX it is more convenient to consider a local, TX-RX centered CCS $(x, y, z)$. Therefore, we use a local right-handed coordinate system with the $z$-axis running from TX to RX, the $y$-axis pointing towards the ground in the ENU coordinate frame, and the origin located in the middle between TX and RX, see Fig. 1. The advantage of the TX-RX-centric description is an analytical tractability of the resulting models. However, the price for this tractability is a general time dependency of the local coordinate frame for objects that are stationary in the global coordinate system. Due to the movement of TX and $\mathrm{RX}$ in the non-moving ENU frame, the orientation and the origin of the local coordinate system change with respect to the fixed ENU coordinate system. In the following, we keep this time dependency of the local coordinate system implicit to simplify the notation. 


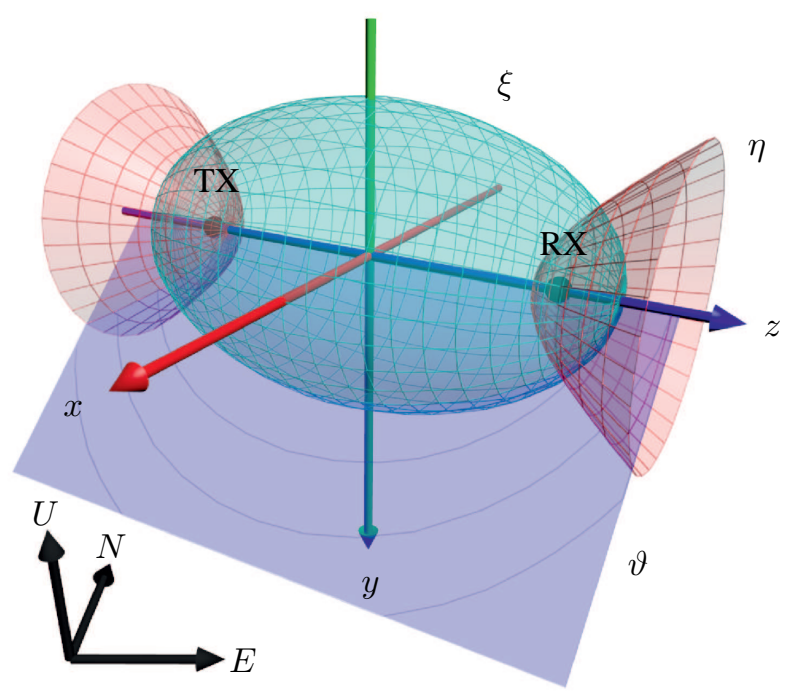

Fig. 1. A local prolate spheroidal coordinate system with the surfaces of constant $\xi$ (ellipsoid), $\eta$ (hyperboloid), and $\vartheta$ (half-plane). The TX and RX are in the foci of the ellipsoids and hyperboloids. A fixed, ground-based ENU coordinate system is shown to relate the fixed and the moving coordinate systems.

We define the location of TX and RX to be the foci of the PSCS. The transformation between local Cartesian coordinates $(x, y, z)$ and PSCs $(\xi, \eta, \vartheta)$ can be computed using the relationships in [34]

$$
\begin{aligned}
& x=l \sqrt{\left(\xi^{2}-1\right)\left(1-\eta^{2}\right)} \cos \vartheta, \\
& y=l \sqrt{\left(\xi^{2}-1\right)\left(1-\eta^{2}\right)} \sin \vartheta, \\
& z=l \xi \eta,
\end{aligned}
$$

where $\xi \in[1, \infty), \eta \in[-1,1], \vartheta \in[0,2 \pi)$ are shown in Fig. 1 and $l$ in (1) is the focus distance of both TX and RX to the origin of the coordinate system. The coordinate $\xi$ plays a special role in the PSC description, since $\xi$ defines a set of points - an ellipsoid - that has a constant propagation delay $\tau$ between TX, scatterers, and RX. Thus, this coordinate has a direct physical interpretation as normalized propagation delay $\xi=\tau / \tau_{\text {los }}$, where $\tau_{\text {los }}$ is the line-of-sight (LOS) propagation delay. Obviously, scattering can only take place if $\xi>1$, since $\xi=1$ corresponds to the LOS path.

Let $d_{\mathrm{t}}(t, \xi, \eta)$ be the distance from TX to an arbitrary scatterer at location $(\xi, \eta, \vartheta)$ and at time $t$ in the local PSCS, and let $d_{\mathrm{r}}(t, \xi, \eta)$ be the corresponding distance from this scatterer to the RX. In PSCs, we obtain

$$
d_{\mathrm{t}}(t, \xi, \eta)=(\xi+\eta) l, \quad \text { and } \quad d_{\mathrm{r}}(t, \xi, \eta)=(\xi-\eta) l,
$$

where the total distance $d_{\mathrm{sc}}(t, \xi)$ from TX via scatterer to RX in PSC is calculated as $d_{\mathrm{sc}}(t, \xi)=d_{\mathrm{t}}(t, \xi, \eta)+d_{\mathrm{r}}(t, \xi, \eta)=$ $2 \xi l$. Thus, the total distance $d_{\mathrm{sc}}(t, \xi)-$ or total scattering delay $\tau_{\mathrm{sc}}=d_{\mathrm{sc}}(t, \xi) / c$, with $c$ being the speed of light - from TX to RX of a scattered signal only depends on the $\xi$-coordinate in the PSCS.
Due to the movement of the TX and RX and the geometric orientation of the scattering plane, the Doppler frequency of the received signal in general varies in time. The time-variant or instantaneous Doppler frequency is calculated according to [21] as

$$
\begin{aligned}
& f_{\mathrm{d}}(t, \xi, \eta, \vartheta)=\frac{f_{\mathrm{c}}}{c}( \\
& \frac{\xi \eta+1}{\xi+\eta} v_{\mathrm{t} z}+\frac{\sqrt{\left(\xi^{2}-1\right)\left(1-\eta^{2}\right)}}{\xi+\eta}\left(v_{\mathrm{t} x} \cos \vartheta+v_{\mathrm{t} y} \sin \vartheta\right) \\
&\left.+\frac{\xi \eta-1}{\xi-\eta} v_{\mathrm{r} z}+\frac{\sqrt{\left(\xi^{2}-1\right)\left(1-\eta^{2}\right)}}{\xi-\eta}\left(v_{\mathrm{r} x} \cos \vartheta+v_{\mathrm{r} y} \sin \vartheta\right)\right)
\end{aligned}
$$

where $\mathbf{v}_{\mathrm{t}}=\left[v_{\mathrm{t} x}, v_{\mathrm{t} y}, v_{\mathrm{t} z}\right]^{\mathrm{T}}$ and $\mathbf{v}_{\mathrm{r}}=\left[v_{\mathrm{r} x}, v_{\mathrm{r} y}, v_{\mathrm{r} z}\right]^{\mathrm{T}}$ are the velocity vectors of TX and RX in the local CCS, respectively. The delay-dependent - also known as isorange in the radar literature - Doppler frequency is obtained by fixing the coordinate $\xi$ to an arbitrary value $\xi=\xi^{*}$.

The expression in (3) is the key for deriving both the delay-dependent Doppler and the joint delay Doppler pdfs. We proceed by postulating a random distribution of scatterers. The corresponding Doppler distribution is then obtained by a probability density transformation through the nonlinear function in (3). Subsequently, we show how these computations are carried out for both the delay-dependent Doppler pdf and the joint delay Doppler pdf.

In order to make the derivation of the pdfs tractable, we introduce some constraints. In the following, we assume that scatterers lie on a fixed plane - the scattering plane - which is arbitrarily oriented in space. Constraining the analysis to this scattering plane is, on the one hand, physically motivated, since in some $\mathrm{V} 2 \mathrm{~V}$ or $\mathrm{A} 2 \mathrm{~A}$ scenarios all scatterers are located on the ground. On the other hand, such an approach simplifies the derivation and consequent analysis of the resulting pdf expressions for general M2M scenarios.

In the local CCS the scattering plane is defined as

$$
A x+B y+C z=l D,
$$

where the four parameters $\{A, B, C, D\} \in \mathbb{R}$ determine its orientation in space. For our purposes we cast the scattering plane in (4) in PSCs, which results in

$$
\begin{aligned}
A l \sqrt{\left(\xi^{2}-1\right)\left(1-\eta^{2}\right)} \cos \vartheta & +B l \sqrt{\left(\xi^{2}-1\right)\left(1-\eta^{2}\right)} \sin \vartheta \\
& +C l \xi \eta=l D .
\end{aligned}
$$

Note that we scaled the distance to the origin of the scattering plane on the right hand side with the focus distance $l$ in (4) and (5) to simplify the subsequent derivations and keep the parameter $D$ of the scattering plane dimensionless.

The scattering plane as any 2D plane embedded in 3D space can be parameterized by two independent variables in the selected coordinate system. Our goal is to obtain a parameterization that allows for an algebraic analysis of the Doppler frequency. Since we need $\xi$ for a delay-dependent description, we can choose either $\eta$ or $\vartheta$ as second variable. In fact, we need both the $(\xi, \eta)$ and $(\xi, \vartheta)$ parameterizations to cover all possible scattering planes in 3D space. Our main parameterization is, however, the one with the $(\xi, \eta)$-coordinates, 
since it allows an algebraic analysis of the Doppler frequency with the help of the algebraic curve theory. The remaining scattering planes, which cannot be parameterized by $(\xi, \eta)$, since they are orthogonal to the $z$-axis in the local CCS, are described by the $(\xi, \vartheta)$-coordinates. It complements the $(\xi, \eta)$ parameterization and allows an algebraic analysis even without using the algebraic curve theory. In the following, we refer to these cases as general case and complementary case, respectively.

In order to derive both the delay-dependent Doppler pdf and the joint delay Doppler pdf, two very similar strategies are followed. Subsequently, we outline the approaches on a general level to give the reader a global perspective on the methodology.

In case of the delay-dependent Doppler pdf, the normalized delay $\xi$ and thus the delay ellipsoid is fixed. The scatterers lie on an ellipse created from the intersection of the scattering plane and the delay ellipsoid. We refer to this intersection ellipse using the implicit equation $q_{\xi}(\eta, \vartheta)=0$ for the general description, which simplifies to $q_{\xi}(\eta)=0$, if the parameterization is in $(\xi, \eta)$-coordinates or $q_{\xi}(\vartheta)=0$, if the parameterization is in $(\xi, \vartheta)$-coordinates. Furthermore, we treat all scatterers lying on $q_{\xi}(\eta, \vartheta)=0$ as identical and uniformly distributed along the curve. As such, the density $s$ of scatterers along the intersection ellipse can be modeled as

$$
p\left(t, q_{\xi}(\eta, \vartheta) ; s \mid \xi\right)=\frac{1}{L},
$$

where $L$ is the length of the intersection curve. We note the semicolon notation in $p(\cdot ; \cdot)$, which is used to separate deterministic variables to the left of the semicolon from the random variables to the right of the semicolon. Thus, the notation indicates the deterministic dependence on time and on the scattering plane of the obtained pdfs.

The delay-dependent Doppler pdf is obtained by transforming the spatial distribution of scatterers into a delay-dependent Doppler frequency distribution using (3) and following standard rules of probability transformation according to [35] as

$$
p\left(t, q_{\xi}(\eta, \vartheta) ; f_{\mathrm{d}} \mid \xi\right)=p\left(t, q_{\xi}(\eta, \vartheta) ; s \mid \xi\right)\left|\mathbf{J}_{s}^{-1}\right|,
$$

where $\mathbf{J}_{\mathbf{s}}{ }^{-1}=\mathrm{d} s / \mathrm{d} f_{\mathrm{d}}$ is the inverse $1 \times 1$ Jacobian matrix of the transformation.

In order to derive the joint delay Doppler pdf we proceed similarly and restrict our analysis to scatterers lying on the scattering plane. In this case, however, we consider scatterers that lie on the portion of the scattering plane circumscribed by the intersection ellipse. This scattering ellipse can be generally described by the implicit expression $q(\xi, \eta, \vartheta)=0$, which simplifies to $q(\xi, \eta)=0$, if the parameterization is in $(\xi, \eta)$ coordinates or $q(\xi, \vartheta)=0$, if the parameterization is in $(\xi, \vartheta)$ coordinates. The resulting object - effectively the area inside the ellipse where the scatterers are located - is then used similarly to the intersection ellipse $q_{\xi}(\eta, \vartheta)=0$ computed in the delay-dependent Doppler pdf case. We assume that the scatterers lying within $q(\xi, \eta, \vartheta)=0$ are identical and uniformly distributed. Thus, the two-dimensional density $\mathbf{s}$ of the scatterers is modeled as

$$
p(t, q(\xi, \eta, \vartheta) ; \mathbf{s})=\frac{1}{\mathcal{Y}},
$$

where $\mathcal{Y}$ is the equivalent area of the ellipse $q(\xi, \eta, \vartheta)=0$.

The joint delay Doppler pdf is then obtained by transforming the distribution of scatterers $\mathbf{s}$ into $\left(\xi, f_{\mathrm{d}}\right)$-coordinates using (3) and rules of probability transformation as

$$
p\left(t, q(\xi, \eta, \vartheta) ; \xi, f_{\mathrm{d}}\right)=p(t, q(\xi, \eta, \vartheta) ; \mathbf{s})\left|\mathbf{J}_{\mathbf{s}}{ }^{-1}\right|,
$$

where $\mathbf{J}_{\mathbf{s}}{ }^{-1}$ is the inverse $2 \times 2$ Jacobian matrix of the variable transformation.

For both the delay-dependent Doppler and the joint delay Doppler pdf, the transformation from the spatial domain to the Doppler domain, i.e., $s \mapsto f_{\mathrm{d}}$ or $\mathbf{s} \mapsto\left(\xi, f_{\mathrm{d}}\right)$ introduces ambiguities in the mapping. These ambiguities, however, can be resolved by applying the algebraic curve theory to the Doppler frequency description. Furthermore, the locations of the extrema and thus the limiting frequencies of the pdfs can be determined.

\section{B. Doppler Frequency as Algebraic Curve}

A plane algebraic curve defines a set of points that are the zeros of a polynomial in two variables (see e.g., [36] or [37]), i.e., an algebraic curve is a one-dimensional algebraic variety. Since we only need the algebraic curve description in $(\xi, \eta)$ coordinates, we provide the definitions in these coordinates. In our case, given a fixed delay $\xi=\xi^{*}$ we can interpret the intricate relationship between the Doppler frequency $f_{\mathrm{d}}$ and $\eta$ as an algebraic curve defined by

$$
\mathcal{C}_{J I}:=V(F)=\left\{\left(\eta, f_{\mathrm{d}}\right) \in[-1,1] \times \mathbb{R} \mid F\left(\eta, f_{\mathrm{d}}\right)=0\right\} .
$$

with the algebraic variety $V(F)$ and $F\left(\eta, f_{\mathrm{d}}\right)$ being a polynomial in two variables with the maximum degree of $n=$ $J+I=\max \left\{j+i \mid a_{j i} \neq 0\right\}$ defined as

$$
F\left(\eta, f_{\mathrm{d}}\right)=\sum_{j+i \leq n} a_{j i} \eta^{j} f_{\mathrm{d}}^{i},
$$

with $a_{j i} \in \mathbb{R}$ being the coefficients of the polynomial and $n$ being the degree of the curve according to [38]. In order to obtain a unique mapping from $s \mapsto f_{\mathrm{d}}$ or $\mathbf{s} \mapsto\left(\xi, f_{\mathrm{d}}\right)$, the polynomial can be solved for $f_{\mathrm{d}}$.

For the determination of the limiting frequencies, i.e., maximum and minimum frequency of the pdfs, the representation as an algebraic curve helps to seperate extrema from singular points. An algebraic curve possesses singular points at locations where $\left[\partial F / \partial \eta, \partial F / \partial f_{\mathrm{d}}\right]=0$, see also [17]. According to [36], for an arbitrary curve $\mathcal{C}_{J I}$ the singular points are defined as

$$
\begin{aligned}
& \operatorname{SING}\left(\mathcal{C}_{J I}\right):=\left\{\left(\eta, f_{\mathrm{d}}\right) \in[-1,1] \times \mathbb{R} \mid F\left(\eta, f_{\mathrm{d}}\right)=0,\right. \\
& \left.\frac{\partial F\left(\eta, f_{\mathrm{d}}\right)}{\partial \eta}=\frac{\partial F\left(\eta, f_{\mathrm{d}}\right)}{\partial f_{\mathrm{d}}}=0\right\}
\end{aligned}
$$

In other words, $\operatorname{SING}\left(\mathcal{C}_{J I}\right)=\mathcal{C}_{J I} \cap \mathcal{C}_{J I}^{\eta} \cap \mathcal{C}_{J I}^{f_{\mathrm{d}}}$, where we define $\mathcal{C}_{J I}^{\eta}:=V(\partial F / \partial \eta)$ and $\mathcal{C}_{J I}^{f_{\mathrm{d}}}:=V\left(\partial F / \partial f_{\mathrm{d}}\right)$. Note that this set is finite for an algebraic curve [37]. 


\section{Length and Area Calculation with Differential Forms}

Finally, we need to compute lengths and areas of surfaces in curvilinear coordinates in order to normalize the pdfs. Therefore, we use differential forms, which are introduced subsequently.

According to [33] a differential form can be simply seen as an integrand. With differential forms the concepts of gradient, divergence, and rotation are extended to higher dimensions. In the PSCS, we use differential forms to obtain the integrands, i.e., the differential length or area in PSCs [39] that are needed to normalize the pdfs in (6) and (8). For the calculation of the normalization constant for the delay-dependent Doppler pdf, we use differential 1-forms, whereas for the joint delay Doppler normalization constant, we use differential 2-forms.

Following the general approach, we first need to calculate the length of the scattering ellipse as normalization constant for the delay-dependent pdf. To this end, consider the differential length or 1-form of an ellipse segment in PSCs. The nonlinear 1-form $\mathrm{d} s$ is generally calculated as

$$
\mathrm{d} s=\sqrt{\mathrm{d} x^{2}+\mathrm{d} y^{2}+\mathrm{d} z^{2}}=\sqrt{h_{\xi}^{2} \mathrm{~d} \xi^{2}+h_{\eta}^{2} \mathrm{~d} \eta^{2}+h_{\vartheta}^{2} \mathrm{~d} \vartheta^{2}},
$$

with the scale factors [40]

$$
\begin{aligned}
& h_{\xi}=l \sqrt{\frac{\xi^{2}-\eta^{2}}{\xi^{2}-1}}, \quad h_{\eta}=l \sqrt{\frac{\xi^{2}-\eta^{2}}{1-\eta^{2}}}, \\
& h_{\vartheta}=l \sqrt{\left(\xi^{2}-1\right)\left(1-\eta^{2}\right)} .
\end{aligned}
$$

Note that the differential length of an ellipse segment depends in general on the three differentials $\mathrm{d} \xi, \mathrm{d} \eta$ and $\mathrm{d} \vartheta$, but can be reduced to one variable along which we can integrate. In order to compute the total length of the intersection ellipse a path integral in PSCs must be evaluated. Although this is challenging in general, this computation can be simplified in our case. Despite the fact that the scattering plane is oriented in $3 \mathrm{D}$ space, the intersection ellipse is actually a $2 \mathrm{D}$ object, i.e., it only depends on two coordinates. Moreover, for a fixed $\xi=$ $\xi^{*}$, the corresponding dependency becomes one-dimensional. As mentioned before, depending on the orientation of the scattering plane we can distinguish two parameterizations, where the spatial distribution of the scatterers either depends only on $(\xi, \eta)$ or on $(\xi, \vartheta)$, respectively.

Thus, the normalization constant for the delay-dependent Doppler pdf in (6) becomes

$$
L=\oint_{q_{\xi}(\eta, \vartheta)=0} \mathrm{~d} s
$$

where $q_{\xi}(\eta, \vartheta)$ is the parameterization-independent definition of the ellipse, which means the integral is either integrated over $\eta$ or $\vartheta$.

In order to calculate the area in PSCs, we need to transform the differentials of the coordinates from the local CCS to the PSCS. The differentials transform with the help of the Jacobian matrix $\mathbf{J}$ according to [41] as

$$
[\mathrm{d} x, \mathrm{~d} y, \mathrm{~d} z]^{\mathrm{T}}=\mathbf{J}[\mathrm{d} \xi, \mathrm{d} \eta, \mathrm{d} \vartheta]^{\mathrm{T}}
$$

$$
\left(\begin{array}{l}
\mathrm{d} x \\
\mathrm{~d} y \\
\mathrm{~d} z
\end{array}\right)=\left(\begin{array}{lll}
\frac{\partial x}{\partial \xi} & \frac{\partial x}{\partial \eta} & \frac{\partial x}{\partial \vartheta} \\
\frac{\partial y}{\partial \xi} & \frac{\partial y}{\partial \eta} & \frac{\partial y}{\partial \vartheta} \\
\frac{\partial z}{\partial \xi} & \frac{\partial z}{\partial \eta} & \frac{\partial z}{\partial \vartheta}
\end{array}\right)\left(\begin{array}{c}
\mathrm{d} \xi \\
\mathrm{d} \eta \\
\mathrm{d} \vartheta
\end{array}\right)
$$

The differential area $\mathrm{d} S$ of an arbitrarily oriented plane, which is a nonlinear 2-form, is calculated according to [33] as

$$
\mathrm{d} S=\sqrt{(\mathrm{d} x \wedge \mathrm{d} y)^{2}+(\mathrm{d} y \wedge \mathrm{d} z)^{2}+(\mathrm{d} z \wedge \mathrm{d} x)^{2}}
$$

where $\wedge$ is the wedge product defined as

$$
\lambda \wedge \mu=(-1)^{k p} \mu \wedge \lambda
$$

with $\lambda$ being a $k$-form and $\mu$ an $p$-form. The wedge product is by construction alternating, i.e., $\mathrm{d} x \wedge \mathrm{d} y=-\mathrm{d} y \wedge \mathrm{d} x$ and $\mathrm{d} x \wedge \mathrm{d} x=0$ for two differential 1-forms. The total area of a surface is calculated as [33]

$$
S=\int_{R} g(x, y, z) \sqrt{(\mathrm{d} x \wedge \mathrm{d} y)^{2}+(\mathrm{d} y \wedge \mathrm{d} z)^{2}+(\mathrm{d} z \wedge \mathrm{d} x)^{2}},
$$

where the integral over $g(x, y, z)$ calculates the surface area of a graph over the region $R:=q(\xi, \eta, \vartheta)=0$. The region is bounded by $\xi=\xi_{\max }$, which corresponds to the maximum delay that we are interested in.

For the scattering plane $q(\xi, \eta, \vartheta)$, the area calculation in (17) can be computed using three different equations as

$$
S= \begin{cases}\int_{R} \sqrt{\left(\frac{\partial g_{1}}{\partial x}\right)^{2}+\left(\frac{\partial g_{1}}{\partial y}\right)^{2}+1}|\mathrm{~d} x \wedge \mathrm{d} y| & \text { if } C \neq 0, \\ \int_{R} \sqrt{\left(\frac{\partial g_{2}}{\partial y}\right)^{2}+\left(\frac{\partial g_{2}}{\partial z}\right)^{2}+1}|\mathrm{~d} y \wedge \mathrm{d} z| & \text { if } A \neq 0, \\ \int_{R} \sqrt{\left(\frac{\partial g_{3}}{\partial z}\right)^{2}+\left(\frac{\partial g_{3}}{\partial x}\right)^{2}+1}|\mathrm{~d} z \wedge \mathrm{d} x| & \text { if } B \neq 0,\end{cases}
$$

depending on the representation of the scattering plane in (4). The plane can be implicitly represented with $z=g_{1}(x, y)$, $x=g_{2}(y, z)$ or $y=g_{3}(z, x)$. In the following, the three square roots are referred as to $r_{i}$ and the differential areas $\mathrm{d} S_{i}$ are special cases of (17). Finally, for computing the joint delay Doppler pdf in (8) we compute the weighted area $\mathcal{Y}$ of the intersection as

$$
\mathcal{Y}=\int_{R} w r_{i} \mathrm{~d} S_{i}
$$

where $r_{i}, i \in\{1,2,3\}$, is the square root term in (20) selected according to one of the chosen parameterizations of the scattering plane $g_{i}$. The differential area $\mathrm{d} S_{i}$ either consists of $|\mathrm{d} x \wedge \mathrm{d} y|$ or $|\mathrm{d} y \wedge \mathrm{d} z|$ or $|\mathrm{d} z \wedge \mathrm{d} x|$ depending on the scattering plane parameterization. The path loss inspired weighting function is given by $w$.

\section{DELAY-DEPENDENT DopPleR FunCTIONS}

In the following, we derive the delay-dependent Doppler pdf for both the general case and the complementary case of the scattering plane. Furthermore, the delay-dependent limiting Doppler frequency is calculated. 


\section{A. General Delay-Dependent Doppler PDF}

We begin by considering all orientations of the scattering plane except the complementary case when the scattering plane is orthogonal to the $z$-axis. It follows that for the general case the parameters of the scattering plane are such that $A \neq 0$ or $B \neq 0$. As we have shown in [17], the differential length of an ellipse segment according to (13) on the scattering plane simplifies for the $(\xi, \eta)$ parameterization to

$$
\begin{aligned}
\mathrm{d} s= & l \sqrt{\frac{\xi^{2}-\eta^{2}}{1-\eta^{2}}+\left(\xi^{2}-1\right)\left(1-\eta^{2}\right)\left(\frac{\partial \vartheta}{\partial \eta}\right)^{2}} \mathrm{~d} \eta= \\
& l \sqrt{\frac{\left(\xi^{2}-1\right)\left(\left(A^{2}+B^{2}\right)\left(\xi^{2}-\eta^{2}\right)-\left(D^{2}-\xi^{2} C^{2}\right)\right)-(D-C \xi \eta)^{2}}{\left(\xi^{2}-1\right)\left(1-\eta^{2}\right)\left(A^{2}+B^{2}\right)-(D-C \xi \eta)^{2}}} \mathrm{~d} \eta .
\end{aligned}
$$

The variable $\vartheta$ for the scattering plane is expressed as a function of the other two coordinates as $\vartheta(\xi, \eta)$. To this end, we rearrange (5) and solve it for $\vartheta$ as

$$
\vartheta_{k}= \pm \arccos (\alpha \pm \beta),
$$

where $\alpha$ and $\beta$ are calculated according to (24). There are four possible solutions: $\vartheta_{1}=\arccos (\alpha+\beta), \vartheta_{2}=\arccos (\alpha-\beta)$, $\vartheta_{3}=-\arccos (\alpha+\beta)$, and $\vartheta_{4}=-\arccos (\alpha-\beta)$. This ambiguity arises due to the presence of the trigonometric functions in the expression of the scattering plane in PSCs.

We proceed by inserting (23) into (3), which for a fixed $\xi=\xi^{*}$ results in an expression for the Doppler frequency for scatterers lying on the intersection ellipse. By inspecting the result, we can conclude that there are four expressions for the Doppler frequency that depend on the coordinate $\eta$, see also Fig. 2. These expressions essentially define four separate, yet, connected segments or branches of a closed curve in $\left(\eta, f_{\mathrm{d}}\right)$ coordinates. The obtained solutions, however, are ambiguous due to the presence of square root and double square root expressions in the Doppler frequency. This ambiguity can be resolved by interpreting the Doppler frequency as an algebraic curve, as discussed subsequently.

Before being able to compute the length of the scattering ellipse, we need to determine the domain of the mapping $\eta \mapsto f_{\mathrm{d}}$, i.e., the range of values for the $\eta$-coordinate that results when an arbitrarily oriented scattering plane with $A \neq 0$ or $B \neq 0$ intersects the delay ellipsoid. We do this by considering (23) and (24). Specifically, we set $\beta=0$ in (24) and solve it for the variable $\eta$. Using the fact that the solution describes an ellipse, the range of possible values for $\eta$ can be computed in closed form. The result is given by the range $\left[\eta_{\min }(\xi), \eta_{\max }(\xi)\right]$ in (25).

As we see from (25) the resulting expression is a function of $\xi$. For an arbitrarily oriented scattering plane there is a minimum delay $\xi_{\text {sr }}$ which results in the specular reflection off the plane. The value of $\xi_{\mathrm{sr}}$ is computed by finding the delay $\xi$ for which $\eta_{\min }=\eta_{\max }=\eta_{\mathrm{sr}}$. This is equivalent to setting the square root in (25) to zero. It can be shown that

$$
\xi_{\mathrm{sr}}=\max \left(\sqrt{\frac{A^{2}+B^{2}+D^{2}}{A^{2}+B^{2}+C^{2}}}, 1\right) .
$$

Let us study the result in (26) in more detail. Note that when $D^{2}<C^{2}$ the scattering plane intersects the LOS and, thus, blocks the signal path. When $\xi_{\text {sr }}=1$, i.e., $|D|=|C|$, the scattering plane includes either TX or RX and $\left|\eta_{\mathrm{sr}}\right|=1$; for these two cases the specular reflection (SR) component coincides with the LOS component. Thus, the span of possible delay values for scattering is given by $\xi>\xi_{\mathrm{sr}}$.

If $A=0$, we obtain a special symmetrical case, which occurs for A2A channels with two communicating aircraft. In this case, the intersection ellipse is cut into two symmetrical parts by the $y$ - $z$-plane containing the aircraft. This scenario has been investigated in [17] and the results we obtain here generalize those in [17]. If we consider the case $A=C=D=0$ and $B=1$, the scattering plane coincides with the $x$-zplane. This alignment simplifies the equations even further. This particular case is equivalent to the $2 \mathrm{D} \mathrm{V} 2 \mathrm{~V}$ channel model discussed in [30], which is again covered by the general equations that we obtain in this paper.

The analysis of the Doppler frequency in (3) after inserting (23) for the general case leads to expressions that contain polynomials and square roots. The computation of the Doppler pdf is not possible due to the ambiguous mapping $\eta \mapsto f_{\mathrm{d}}$. Although trigonometric functions were replaced by rational expressions, the resulting expression is still ambiguous as it contains multiple square root quantities. Nonetheless, the corresponding ambiguities can be resolved by applying the algebraic curve theory.

Indeed, the algebraic curve as defined in (10) can be derived from (3) after inserting (23) and (24), appropriate re-arrangement, and two squaring operations. The steps for computing the unique algebraic curve are summarized in Alg. 1.

Following the steps $1-4$ of the algorithm, we obtain a 12th degree curve $\mathcal{C}_{84}$ with the highest monomial $\eta^{8} f_{\mathrm{d}}^{4}$. Thus, the resulting degree of the curve is twice as high as the one obtained in [17] for A2A scenarios. This is due to the above mentioned ambiguity of the Doppler curves; thus it can be shown that this increase of the degree is caused merely by the ambiguity when replacing the trigonometric functions (steps 3 and 4 of Alg. 1). Nonetheless, the algebraic curve $\mathcal{C}_{84}$ can be factored as $\mathcal{C}_{84}=\mathcal{C}_{42}^{\prime} \cup \mathcal{C}_{42}^{\prime \prime}$ (step 5 of Alg. 1) according to Theorem 1.13 in [37]. One of the two curves corresponds to the actual Doppler frequency caused by the correct scattering plane given by (5); the other one corresponds to the Doppler

$$
\begin{gathered}
\alpha=\frac{A(D-C \xi \eta)}{\sqrt{\left(\xi^{2}-1\right)\left(1-\eta^{2}\right)}\left(A^{2}+B^{2}\right)}, \beta=\frac{B \sqrt{\left(\xi^{2}-1\right)\left(1-\eta^{2}\right)\left(A^{2}+B^{2}\right)-(D-C \xi \eta)^{2}}}{\sqrt{\left(\xi^{2}-1\right)\left(1-\eta^{2}\right)}\left(A^{2}+B^{2}\right)} \\
\eta_{\min , \max }(\xi)=\frac{D C \xi \pm \sqrt{D^{2} C^{2} \xi^{2}-\left(A^{2} \xi^{2}+B^{2} \xi^{2}+C^{2} \xi^{2}-A^{2}-B^{2}\right)\left(A^{2}+B^{2}+D^{2}-A^{2} \xi^{2}-B^{2} \xi^{2}\right)}}{A^{2} \xi^{2}+B^{2} \xi^{2}+C^{2} \xi^{2}-A^{2}-B^{2}}
\end{gathered}
$$



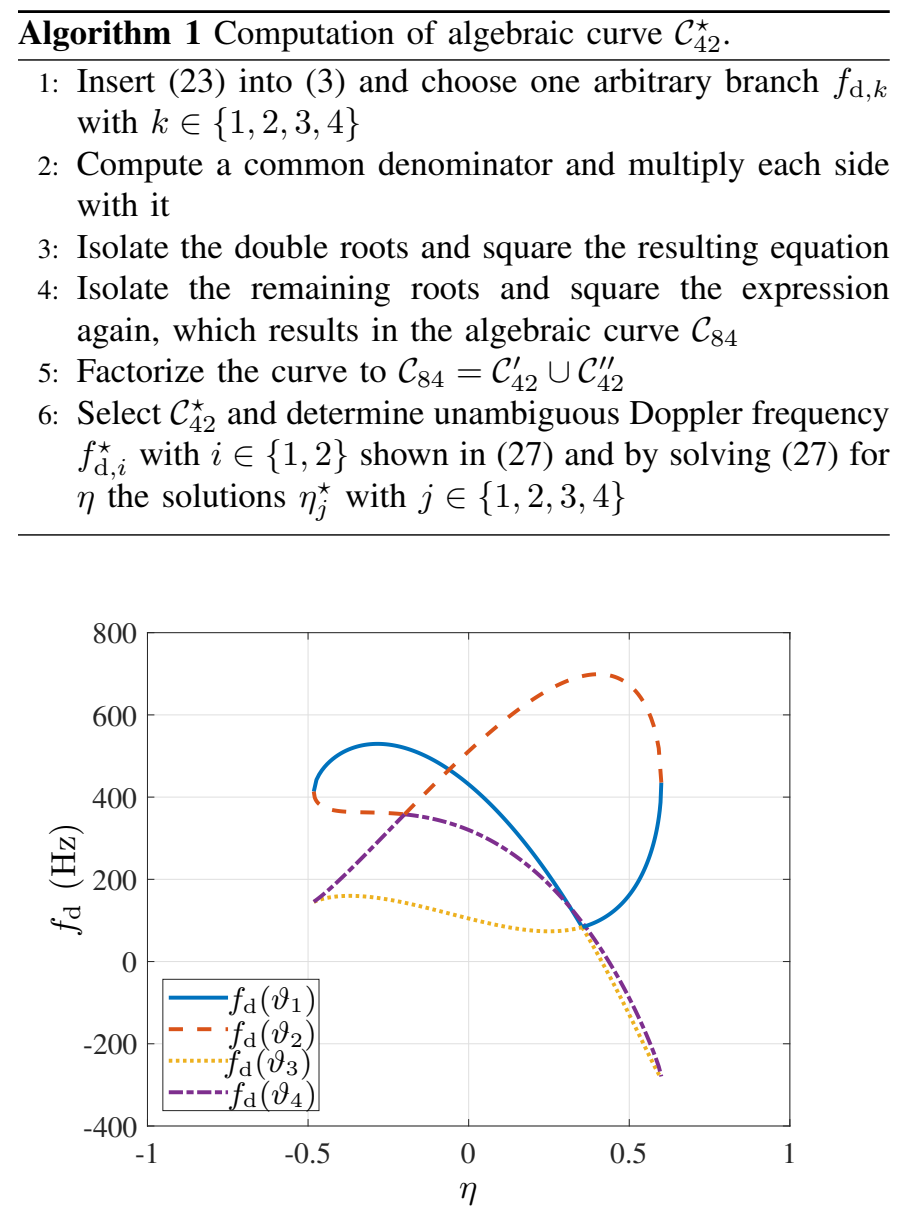

Fig. 2. Ambiguous delay-dependent Doppler algebraic curve $\mathcal{C}_{84}=\mathcal{C}_{42}^{\prime} \cup \mathcal{C}_{42}^{\prime \prime}$ due to the ambiguity for $A=-1, B=2, C=3, D=1 / 2, \xi=2, f_{\mathrm{c}}=$ $5.2 \mathrm{GHz}, \mathbf{v}_{\mathrm{t}}=[70,-30,60]^{\mathrm{T}} \mathrm{km} / \mathrm{h}$ and $\mathbf{v}_{\mathrm{r}}=[-90,100,-80]^{\mathrm{T}} \mathrm{km} / \mathrm{h}$. The crunode at $\eta_{\mathrm{s}}=0.3158$ for the correct curve $\mathcal{C}_{42}^{\star}$ is shown.

frequency caused by the scattering plane with the sign of the coefficient $B$ flipped to $-B$, or equivalently to flipping of the signs of $A, C$ and $D$ to $-A,-C,-D$, respectively. The sign of the coefficients vanishes in (24) due to squaring or multiplication. As such, only one of the curves $\mathcal{C}_{42}^{\prime}$ or $\mathcal{C}_{42}^{\prime \prime}$ describes the correct $\eta-f_{\mathrm{d}}$ relationship induced by the scattering plane; the other one can simply be disregarded.

To find the correct curve we need to check, if the points on the curve satisfy the scattering plane equation in (5). In order to check this, we proceed as follows. We pick an arbitrary point on the curve $\mathcal{C}_{42}^{\prime}$ and make sure that this point is not an intersection point with the other curve. Otherwise we won't be able to identify the correct curve. Then, we insert the corresponding values in (5). If the expression is satisfied, the curve $\mathcal{C}_{42}^{\prime}$ corresponds to the scattering plane; otherwise, $\mathcal{C}_{42}^{\prime \prime}$ is the correct solution. In the following, the selected curve is denoted as $\mathcal{C}_{42}^{\star}$. The expression of the correctly selected Doppler frequency is shown in (27). It is important to stress that once $\mathcal{C}_{42}^{\star}$ is found, i.e., the curve is derived as a function of $A, B, C$ and $D$, it remains valid for arbitrarily oriented planes and thus always specifies the correct relationship between $\eta$ and $f_{\mathrm{d}}$ in the general case. Due to the resolution of the ambiguity, it becomes possible to compute the unique Doppler pdf.

To better illustrate the ambiguous curves and the effect of the factorization, we consider an example of a mobile TX and $\mathrm{RX}$, which are neither in the scattering plane like in the $\mathrm{V} 2 \mathrm{~V}$ case nor that the scattering plane is orthogonal to the $y$-z-plane like in the A2A case, but in a more general setting. For this scenario, the ambiguous $\eta$ - $f_{\mathrm{d}}$ algebraic curves $\mathcal{C}_{42}^{\prime}$ and $\mathcal{C}_{42}^{\prime \prime}$ are shown in Fig. 2. Each of the curves has four segments that correspond to four branches of the Doppler frequency curve $k \in\{1,2,3,4\}$ corresponding to (23). One can clearly see that a branch of a certain style on one curve always connects to a branch of the same style on a different curve - a result of the ambiguities in the original Doppler frequency expression.

After the factorization of the curve and determining $\mathcal{C}_{42}^{\star}$, we can state the following.

Theorem 1. For a fixed Doppler frequency $f_{\mathrm{d}}$, there are up to 4 values of $\eta$ that belong to $\mathcal{C}_{42}^{\star}$ and for a fixed variable $\eta$, there are up to 2 values of $f_{\mathrm{d}}$ that belong to $\mathcal{C}_{42}^{\star}$.

Proof. The proof follows directly from Bézout's theorem, see [38]. Indeed, the intersection of a straight line (vertical for a fixed $\eta$ or horizontal for a fixed $f_{\mathrm{d}}$ ) with the algebraic curve $\mathcal{C}_{42}^{\star}$ results in a quadratic polynomial equation in $f_{\mathrm{d}}$ or a fourth-order polynomial equation in $\eta$, respectively. Therefore, the number of intersections is given by the exponent of the respective variable, i.e., four for the intersection with the horizontal line and two for the intersection with the vertical line.

Since we need to compute the inverse mapping $\eta \mapsto f_{\mathrm{d}}$, the above theorem states that the inverse mapping has to consider at most four possible values of $\eta$ for each $f_{\mathrm{d}}$. For the classical fixed-to-mobile channel the number of possible Doppler frequencies for a fixed delay thus reduces to two, as known from the literature [42]. Indeed, from (3) we can see that if the transmitter is stationary, i.e., $\mathbf{v}_{\mathrm{t}}=0$, the two corresponding trigonometric functions disappear, reducing the total number of ambiguities to two.

$$
\begin{aligned}
& f_{\mathrm{d}, i}^{\star}(t, \xi, \eta)=\frac{1}{\left(A^{2}+B^{2}\right)\left(\xi^{2}-\eta^{2}\right)}\left((D-C \xi \eta)\left(A\left(v_{\mathrm{r} x}(\xi+\eta)+v_{\mathrm{t} x}(\xi-\eta)\right)+B\left(v_{\mathrm{r} y}(\xi+\eta)+v_{\mathrm{t} y}(\xi-\eta)\right)\right)\right. \\
& \pm \sqrt{\left(\left(\xi^{2}-1\right)\left(1-\eta^{2}\right)\left(A^{2}+B^{2}\right)-(D-C \xi \eta)^{2}\right)\left(B\left(v_{\mathrm{r} x}(\xi+\eta)+v_{\mathrm{t} x}(\xi-\eta)\right)-A\left(v_{\mathrm{r} y}(\xi+\eta)+v_{\mathrm{t} y}(\xi-\eta)\right)\right)^{2}} \\
& \left.+\left(A^{2}+B^{2}\right)\left(v_{\mathrm{r} z}(\xi \eta-1)(\xi+\eta)+v_{\mathrm{t} z}(\xi \eta+1)(\xi-\eta)\right)\right) \frac{f_{\mathrm{c}}}{c}
\end{aligned}
$$


Now we have all the necessary prerequisites to calculate the Doppler pdf. Following Theorem 1, for each $\eta$ we obtain two possible values of $f_{\mathrm{d}}: f_{\mathrm{d}, 1}^{\star}(\eta)$ and $f_{\mathrm{d}, 2}^{\star}(\eta)$, which are both functions of $\eta$. These two Doppler functions describe the unambiguous relationship between $f_{\mathrm{d}, i}^{\star}$ and $\eta, i \in\{1,2\}$, for each half of the intersection ellipse. Furthermore, we obtain four functions of $\eta$ : $\eta_{1}^{\star}\left(f_{\mathrm{d}}\right), \eta_{2}^{\star}\left(f_{\mathrm{d}}\right), \eta_{3}^{\star}\left(f_{\mathrm{d}}\right)$, and $\eta_{4}^{\star}\left(f_{\mathrm{d}}\right)$, which are functions of $f_{\mathrm{d}}$. These four $\eta$-functions describe the unambiguous relationship between $\eta_{j}^{\star}$ and $f_{\mathrm{d}}, j \in\{1,2,3,4\}$, for each of the four unique intersection ellipse segments.

Consider the parameter $\eta \in\left[\eta_{\min }(\xi), \eta_{\max }(\xi)\right]$ for each of the two Doppler frequency branches. Thus, the total length of the scattering ellipse is calculated as

$L=2 l \int_{\eta_{\min }(\xi)}^{\eta_{\max }(\xi)} \sqrt{\frac{\left(\xi^{2}-1\right)\left(\left(A^{2}+B^{2}\right)\left(\xi^{2}-\eta^{2}\right)-\left(D^{2}-\xi^{2} C^{2}\right)\right)-(D-C \xi \eta)^{2}}{\left(\xi^{2}-1\right)\left(1-\eta^{2}\right)\left(A^{2}+B^{2}\right)-(D-C \xi \eta)^{2}}} \mathrm{~d} \eta$,

where $\eta_{\min }(\xi)$ and $\eta_{\max }(\xi)$ are computed using (25). The spatial pdf for uniformly distributed scatterers along the ellipse is then calculated as

$p\left(t, q_{\xi}(\eta) ; s \mid \xi\right)=\frac{1}{L}=$

$2 l \int_{\eta_{\min }(\xi)}^{\eta_{\max }(\xi)} \sqrt{\frac{\left(\xi^{2}-1\right)\left(\left(A^{2}+B^{2}\right)\left(\xi^{2}-\eta^{2}\right)-\left(D^{2}-\xi^{2} C^{2}\right)\right)-(D-C \xi \eta)^{2}}{\left(\xi^{2}-1\right)\left(1-\eta^{2}\right)\left(A^{2}+B^{2}\right)-(D-C \xi \eta)^{2}}} \mathrm{~d} \eta$

By applying the rules of probability transformation to obtain the delay-dependent Doppler pdf for the general case, and using (22) we compute

$$
\begin{aligned}
& p\left(t, q_{\xi}(\eta) ; f_{\mathrm{d}} \mid \xi\right)=p\left(t, q_{\xi}(\eta) ; s \mid \xi\right)\left|\frac{\mathrm{d} s}{\mathrm{~d} f_{\mathrm{d}}}\right|=\sum_{j=1}^{|\mathcal{H}|} \frac{l}{L} \times \\
& \sqrt{\frac{\left(\xi^{2}-1\right)\left(\left(A^{2}+B^{2}\right)\left(\xi^{2}-\eta_{j}^{\star 2}\right)-\left(D^{2}-\xi^{2} C^{2}\right)\right)-\left(D-C \xi \eta_{j}^{\star}\right)^{2}}{\left(\xi^{2}-1\right)\left(1-\eta_{j}^{\star 2}\right)\left(A^{2}+B^{2}\right)-\left(D-C \xi \eta_{j}^{\star}\right)^{2}}}\left|\frac{\mathrm{d} \eta_{j}^{\star}}{\mathrm{d} f_{\mathrm{d}}}\right|,
\end{aligned}
$$

with the $\eta_{j}^{\star}\left(f_{\mathrm{d}}\right)$-values lying on the curve $\mathcal{C}_{42}^{\star}$, i.e., $\mathcal{H}=$ $\left\{\eta^{\star} \in \mathcal{C}_{42}^{\star} \mid \eta_{\min }(\xi) \leq \eta^{\star} \leq \eta_{\max }(\xi)\right\}$. The $\eta_{j}^{\star}\left(f_{\mathrm{d}}\right)$-functions are obtained by solving $\mathcal{C}_{42}^{\star}$ for $\eta$ and are unique in the corresponding interval. This functional relationship is shown exemplarily in Fig. 3. There are at most four valid solutions for a given $f_{\mathrm{d}}$ and thus $|\mathcal{H}| \leq 4$, since the curve $\mathcal{C}_{42}^{\star}$ is of degree four in $\eta$. Obviously, only real solutions in the interval $\left[\eta_{\min }(\xi), \eta_{\max }(\xi)\right]$ are of interest.

Note that the focus distance $l$ cancels out in (30) so that the delay-dependent pdf is independent of the actual distance between TX and RX. The reason for this is that the distance of the scattering plane to the origin given by $l D$ is scaled by the focus distance $l$. This means that for all cases, where the relation between focus distance and distance of the scattering plane to the origin is the same, the delay-dependent and the joint delay Doppler pdfs are also the same.

\section{B. Delay-Dependent Limiting Doppler Frequencies}

In the following, we turn our attention to the poles of $p\left(t, q_{\xi}(\eta, \vartheta) ; f_{\mathrm{d}} \mid \xi\right)$, which describe the extrema of the Doppler frequency for arbitrary delays $\xi$. The poles constitute themselves as the zeros of $\partial f_{\mathrm{d}, i}^{\star}(t, \xi, \eta) / \partial \eta=0$ for the general

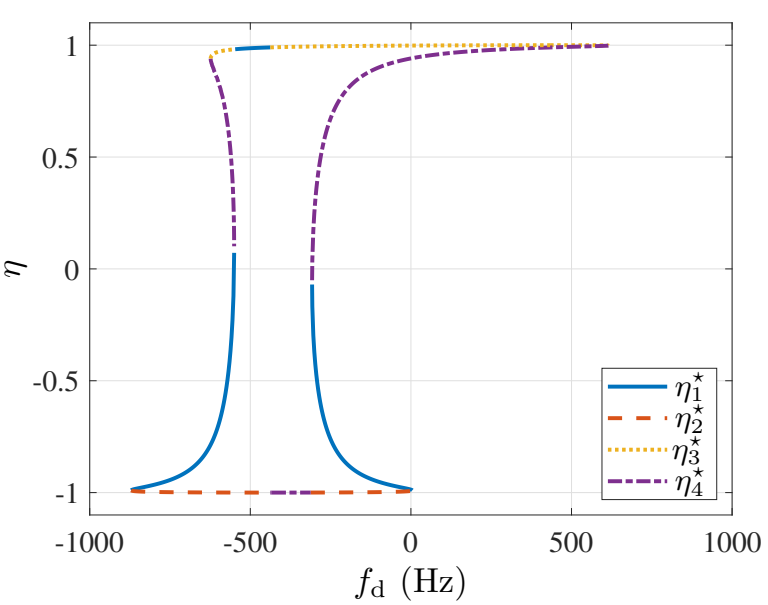

Fig. 3. Segments of the four unique $\eta_{j}^{\star}\left(f_{\mathrm{d}}\right)$ functions for the V2V scenario with $\mathbf{v}_{\mathrm{t}}=[-90,0,0]^{\mathrm{T}} \mathrm{km} / \mathrm{h}, \mathbf{v}_{\mathrm{r}}=[-90,0,90]^{\mathrm{T}} \mathrm{km} / \mathrm{h}$, scattering plane $[0,1,0,0]$, and $\xi=1.01$ from [30].

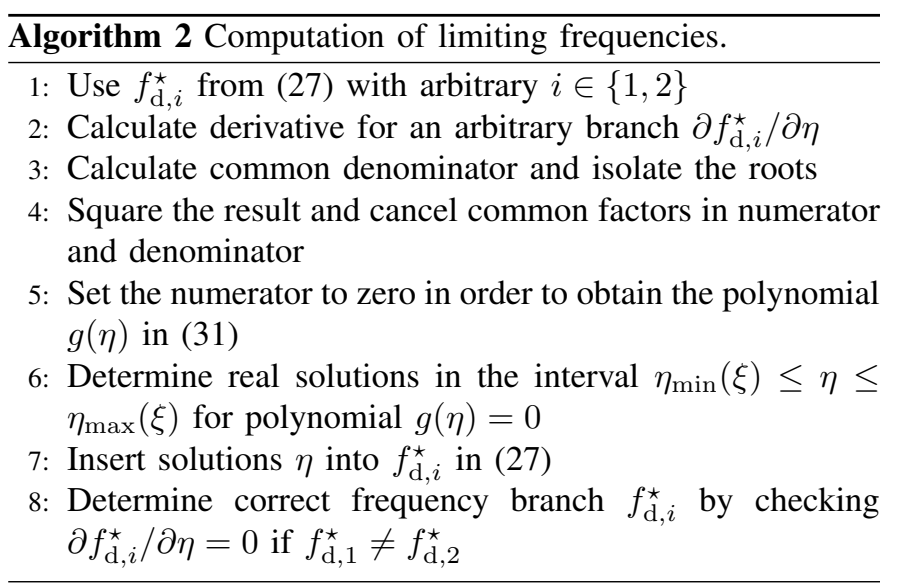

case or $\partial f_{\mathrm{d}}(t, \xi, \vartheta) / \partial \vartheta=0$ for the complementary case. The set of extrema naturally include minimum and maximum Doppler frequencies, since due to the physical limitation of the latter, the corresponding pdf $p\left(t, q_{\xi}(\eta, \vartheta) ; f_{\mathrm{d}} \mid \xi\right)$ must have a finite support. The limiting frequencies are calculated similar to [30] as shown in Alg. 2 by directly computing the derivative of the Doppler frequency. The solution for $\eta$ leads to a delay-dependent sixth order polynomial, which determines the horizontal tangents of the algebraic curve $\mathcal{C}_{42}^{\star}$ as

$$
\begin{aligned}
g(\eta) & =a_{6}\left(\xi^{6}, \xi^{4}, \xi^{2}, \xi^{0}\right) \eta^{6}+a_{5}\left(\xi^{7}, \xi^{5}, \xi^{3}, \xi^{1}\right) \eta^{5} \\
& +a_{4}\left(\xi^{8}, \xi^{6}, \xi^{4}, \xi^{2}, \xi^{0}\right) \eta^{4}+a_{3}\left(\xi^{9}, \xi^{7}, \xi^{5}, \xi^{3}, \xi^{1}\right) \eta^{3} \\
& +a_{2}\left(\xi^{10}, \xi^{8}, \xi^{6}, \xi^{4}, \xi^{2}\right) \eta^{2}+a_{1}\left(\xi^{9}, \xi^{7}, \xi^{5}, \xi^{3}\right) \eta^{1} \\
& +a_{0}\left(\xi^{10}, \xi^{8}, \xi^{6}, \xi^{4}\right) \eta^{0} .
\end{aligned}
$$

The seven coefficients $a_{i}, i=0, \ldots, 6$, are themselves dependent on a total of eleven parameters. The coefficients of the polynomial are too lengthy to be included in the paper; in fact there are 2632 components. Instead, they are made publicly available as supplementary material and ready for use in Code Ocean. They comprise six velocity component coefficients $\mathbf{v}_{\mathrm{t}}=\left[v_{\mathrm{t} x}, v_{\mathrm{t} y}, v_{\mathrm{t} z}\right]^{\mathrm{T}}$ and $\mathbf{v}_{\mathrm{r}}=\left[v_{\mathrm{r} x}, v_{\mathrm{r} y}, v_{\mathrm{r} z}\right]^{\mathrm{T}}$, four scattering plane coefficients $[A, B, C, D]$, and the delay $\xi$. In general, 
roots of (31) need to be determined numerically. However, there are some special cases when the sextic equation allows for an analytical solution according to [43].

Note the particular dependency of the polynomial coefficients in (31) on $\xi$. First of all, it allows a delay-dependent description of the limiting frequencies. We see that even coefficients only possess even exponents of $\xi$ and odd coefficients only odd exponents of $\xi$. Furthermore, only half of the coefficients are independent. Coefficients $a_{i}$ and $a_{6-i}$ are related to each other by replacing the monomials $\xi^{i}$ with $\xi^{10-i}$. Besides, $C$ and $D$ have to be swapped and the signs for certain combinations of the velocity vector components, i.e., $v_{\mathrm{t} x} v_{\mathrm{r} x}$, $v_{\mathrm{t} x} v_{\mathrm{r} y}, v_{\mathrm{t} x} v_{\mathrm{r} z}, v_{\mathrm{t} y} v_{\mathrm{r} x}, v_{\mathrm{t} y} v_{\mathrm{r} y}, v_{\mathrm{t} y} v_{\mathrm{r} z}, v_{\mathrm{t} x} v_{\mathrm{t} z}$, and $v_{\mathrm{t} y} v_{\mathrm{t} z}$ have to be changed. The coefficient $a_{3}$ is symmetric to itself with the above replacements.

The structure of the polynomial, especially the dependency on $\xi$ reveals that for $\xi \rightarrow \infty$ the polynomial becomes a quadratic symmetric polynomial, since the highest exponent of $\xi$ only occurs for $a_{2}$ and $a_{0}$. Thus, the order of the polynomial reduces from six to two in this asymptotic regime. These results generalize those in [30] and [17] for both $\mathrm{V} 2 \mathrm{~V}$ and A2A channels to the general M2M channel.

Theorem 2. The Doppler algebraic curve $\mathcal{C}_{42}^{\star}$ possesses up to six horizontal tangents.

Proof. The theorem follows directly from the fundamental theorem of algebra and the sixth order polynomial in (31).

This theorem is valid for an arbitrarily oriented scattering plane in any single-bounce M2M channel. Thus, both the delay-dependent and the joint delay Doppler frequency probability density functions possess up to six poles.

In addition to the solution of $(31), \mathcal{C}_{42}^{\star}$ possesses singular points as defined in (12). In our previous work singular points were calculated for scattering planes orthogonal to the $y$ $z$-axis. After analyzing the algebraic curve of the Doppler frequency and comparing it with the result in [17], we were able to extend the equation to arbitrary scattering planes.

The singular point for the algebraic Doppler curve of general M2M channels is found for

$$
\eta_{\mathrm{s}}=\frac{v_{\mathrm{ts}}+v_{\mathrm{rs}}}{v_{\mathrm{ts}}-v_{\mathrm{rs}}} \xi=\frac{B v_{\mathrm{t} x}-A v_{\mathrm{t} y}+B v_{\mathrm{r} x}-A v_{\mathrm{r} y}}{B v_{\mathrm{t} x}-A v_{\mathrm{t} y}-B v_{\mathrm{r} x}+A v_{\mathrm{r} y}} \xi,
$$

with

$$
\begin{aligned}
& v_{\mathrm{ts}}=\frac{\mathbf{v}_{\mathrm{t}} \cdot\left(\mathbf{n}_{\mathrm{E}} \times \mathbf{e}_{z}\right)}{\left\|\mathbf{n}_{\mathrm{E}} \times \mathbf{e}_{z}\right\|}=\frac{1}{\sqrt{A^{2}+B^{2}}}\left(\begin{array}{c}
v_{\mathrm{t} x} \\
v_{\mathrm{t} y} \\
v_{\mathrm{t} z}
\end{array}\right) \cdot\left(\begin{array}{c}
B \\
-A \\
0
\end{array}\right), \\
& v_{\mathrm{rs}}=\frac{\mathbf{v}_{\mathrm{r}} \cdot\left(\mathbf{n}_{\mathrm{E}} \times \mathbf{e}_{z}\right)}{\left\|\mathbf{n}_{\mathrm{E}} \times \mathbf{e}_{z}\right\|}=\frac{1}{\sqrt{A^{2}+B^{2}}}\left(\begin{array}{c}
v_{\mathrm{r} x} \\
v_{\mathrm{r} y} \\
v_{\mathrm{r} z}
\end{array}\right) \cdot\left(\begin{array}{c}
B \\
-A \\
0
\end{array}\right),
\end{aligned}
$$

where $\mathbf{n}_{E}=[A, B, C]^{\mathrm{T}}$ is the normal vector of the arbitrarily oriented scattering plane. We calculate the velocity vector components $v_{\mathrm{ts}}$ and $v_{\mathrm{rs}}$ that lie inside the scattering plane and are orthogonal to the $z$-axis. The result in (32) reduces to equation (30) in [17], if the scattering plane is orthogonal to the $y$ - $z$-axis. We state the following result for general M2M channels.
Theorem 3. If $v_{\mathrm{ts}}$ and $v_{\mathrm{rs}}$ have opposite signs, the Doppler algebraic curve $\mathcal{C}_{42}^{\star}$ can possess a singular point at $\eta_{\mathrm{s}}$.

Proof. Since the magnitude of $\eta_{\mathrm{s}}$ has to be smaller than 1 due to $-1 \leq \eta \leq 1$ by definition of the prolate spheroidal coordinate system in (1), $v_{\mathrm{ts}}$ and $v_{\mathrm{rs}}$ need to have opposite signs for $\xi>\xi_{\text {sr }}$ in order to fulfill (32).

In our case, we can distinguish three different types of singular points by following the classification of the singular points used in [36]. In particular, for the $\eta$ - $f_{\mathrm{d}}$ algebraic curve

$$
\left(\frac{\partial^{2} F}{\partial \eta \partial f_{\mathrm{d}}}\right)^{2}\left\{\begin{array}{l}
> \\
= \\
<
\end{array}\right\} \frac{\partial^{2} F}{\partial \eta^{2}} \frac{\partial^{2} F}{\partial f_{\mathrm{d}}^{2}} \begin{aligned}
& \text { crunode } \\
& \text { cusp } \\
& \text { acnode }
\end{aligned}
$$

we obtain a crunode, a cusp, or an acnode, respectively. Exemplary singular points for Doppler frequencies in M2M channels are shown in Fig. 3 of [17].

Note that for all scattering planes with the same parameters $A$ and $B, \eta_{\mathrm{s}}$ is the same for a given $\xi$. Thus, the other two parameters $C$ and $D$ of the scattering plane determine whether we observe a crunode, a cusp or an acnode.

Since singular points are not very common in the channel modeling literature, we explain the meaning of the different singular points with respect to the Doppler frequency curve obtained by the intersection of the delay-dependent Doppler frequency with a scattering plane. A singular point essentially occurs if the Doppler curve crosses itself in the scattering plane due to the geometry. The following cases can be observed:

crunode obtained when the crossing point lies within the scattering plane, i.e, for $\eta_{\min }(\xi)<\eta_{\mathrm{s}}<\eta_{\max }(\xi)$,

cusp obtained when the crossing point is at the border of the scattering plane, i.e, for $\eta_{\mathrm{s}} \in\left\{\eta_{\min }(\xi), \eta_{\max }(\xi)\right\}$,

acnode obtained when the crossing point is found in a plane parallel to the scattering plane, i.e., for $-1 \leq \eta_{\mathrm{s}}<$ $\eta_{\min }(\xi)$ or $\eta_{\max }(\xi)<\eta_{\mathrm{s}} \leq 1$. This means the acnode is an orthogonal projection of the crossing point onto the plane, i.e., it is not a point on the original Doppler frequency $f_{\mathrm{d}, i}^{\star}$, but only occurs on the "squared" algebraic curve $\mathcal{C}_{42}^{\star}$.

Note that $\eta^{\star}$ in (30) is already defined as to include crunodes and cusps, but no acnodes. In degenerate cases, e.g., $v_{\mathrm{t} z} \neq 0$ and $v_{\mathrm{r} z} \neq 0$ or $v_{\mathrm{ts}}=v_{\mathrm{rs}}$, the zeros of (31) turn into singular points. The reason for this is that the algebraic curve becomes reducible, i.e., the curve $\mathcal{C}_{42}^{\star}$ further factors as $\mathcal{C}_{42}^{\star}=\mathcal{C}_{21}^{\star} \cup \mathcal{C}_{21}^{\star}$. We find those points by solving (31), but would fail to classify them as extreme points, since they are boundary values with $\partial f_{\mathrm{d}, i}^{\star} / \partial \eta \neq 0$. Without the algebraic curve theory it could not be explained why the real solutions obtained from (31) are limiting frequencies, even though $\partial f_{\mathrm{d}, i}^{\star} / \partial \eta \neq 0$. The reason that the derivative of a Doppler branch is not zero is the following: these points are singular points or more specifically cusps. For the algebraic curve $\mathcal{C}_{42}^{\star}$, however, the corresponding singular points have a zero derivative. Thus, without the algebraic curve theory, we would fail to calculate correct the limiting frequencies in degenerate cases. 


\section{Complementary Delay-Dependent Doppler PDF}

Let us consider the case when the scattering plane is positioned orthogonal to the $z$-axis. In this configuration the parameters of the scattering plane are $A=0$ and $B=0$. Thus, both (4) and (5) reduce to

$$
C z=l D \text { and } C l \xi \eta=l D,
$$

respectively, and

$$
\eta=\frac{D}{C \xi} .
$$

The intersection ellipse in this case becomes an intersection circle. We consider the value range of the parameters $C$ and $D$ for this case. The relationship between $C$ and $D$ regulates the position of the scattering plane with respect to the $z$-axis. From (37) we see that the minimum delay occurs at

$$
\xi_{\mathrm{sr}}=\max \left(\left|\frac{D}{C}\right|, 1\right),
$$

which is merely a special case of (26). It corresponds to the specular reflection, which is the shortest possible propagation path between TX and RX via the scattering plane. For $\xi_{\text {sr }}=1$ the SR coincides with the LOS. When the geometry of the scattering plane is chosen such that $|D / C|<1$ with $C \neq 0$, the scattering plane would intersect the LOS path and thus block the signal from TX to RX. For the case $|D / C|=1$ the scattering plane would go directly through TX or RX depending on the sign of $\eta$. Finally, when $|D / C|>1$ the scattering plane is clear of the LOS, TX, and RX. The span of possible delay values $\xi$ for scattering, which excludes the specular reflection, is given by the relationship $\xi>\xi_{\text {sr }}$ due to the definition of $\xi$ in (1), which is the same as in the general case.

Subsequently, we determine the differential length of the intersection circle for the complementary case. The general differential length equation in (13) simplifies to

$$
\begin{aligned}
\mathrm{d} s=h_{\vartheta} \mathrm{d} \vartheta & =l \sqrt{\left(\xi^{2}-1\right)\left(1-\eta^{2}\right)} \mathrm{d} \vartheta \\
& =l \sqrt{\left(\xi^{2}-1\right)\left(1-\left(\frac{D}{C \xi}\right)^{2}\right)} \mathrm{d} \vartheta,
\end{aligned}
$$

where we used (37) and the fact that $\mathrm{d} \xi=\mathrm{d} \eta=0$. Thus, the total length of the circle becomes

$$
L=\oint_{q_{\xi}(\vartheta)=0} \mathrm{~d} s=\int_{0}^{2 \pi} l \sqrt{\left(\xi^{2}-1\right)\left(1-\left(\frac{D}{C \xi}\right)^{2}\right)} \mathrm{d} \vartheta .
$$

The pdf of the variable $\vartheta$ for uniformly distributed scatterers on the intersection circle is calculated as follows

$$
p\left(t, q_{\xi}(\vartheta) ; s \mid \xi\right)=\frac{1}{L}=\frac{1}{2 \pi l \sqrt{\left(\xi^{2}-1\right)\left(1-\left(\frac{D}{C \xi}\right)^{2}\right)}} .
$$

The time-variant, delay-dependent Doppler pdf is obtained by a probability transform of (7) between the angle $\vartheta$ and the Doppler frequency $f_{\mathrm{d}}$ given by (3) with $\eta$ defined in (37). To compute the transformation, we express $\vartheta$ as a function of $f_{\mathrm{d}}$ by using the trigonometric identity $a \sin (\vartheta)+b \cos (\vartheta)=$ $\sqrt{a^{2}+b^{2}} \cos (\vartheta-\arctan (a / b))$, and solve (3) for $\vartheta$. Skipping tedious, but straightforward algebraic manipulations, it can be shown that there are two possible solutions $\vartheta_{1}\left(f_{\mathrm{d}}\right)$ and $\vartheta_{2}\left(f_{\mathrm{d}}\right)$ that need to be considered. Using (39) and applying standard rules of probability transformation according to [35], (7) is expressed as

$$
\begin{aligned}
p\left(t, q_{\xi}(\vartheta) ; f_{\mathrm{d}} \mid \xi\right) & =p\left(t, q_{\xi}(\vartheta) ; s \mid \xi\right)\left|\frac{\mathrm{d} s}{\mathrm{~d} f_{\mathrm{d}}}\right| \\
= & \sum_{i=1}^{2} \frac{1}{2 \pi}\left|\frac{\mathrm{d} \vartheta_{i}}{\mathrm{~d} f_{\mathrm{d}}}\right|=\frac{1}{\pi\left|f_{\text {lim }}\right| \sqrt{1-\left(\frac{f_{\mathrm{d}}-f_{\mathrm{o}}}{f_{\mathrm{lim}}}\right)^{2}}},
\end{aligned}
$$

where the offset frequency

$$
f_{\mathrm{o}}(t, \xi)=\frac{f_{\mathrm{c}}}{c}\left(\frac{\frac{D}{C}+1}{\xi+\frac{D}{C \xi}} v_{\mathrm{t} z}+\frac{\frac{D}{C}-1}{\xi-\frac{D}{C \xi}} v_{\mathrm{r} z}\right),
$$

is caused by the movement of TX and RX along the $z$-axis, and the limiting frequency described as

$$
\begin{gathered}
f_{\lim }(t, \xi)= \pm \frac{f_{\mathrm{c}}}{c} \sqrt{\left(\xi^{2}-1\right)\left(1-\left(\frac{D}{C \xi}\right)^{2}\right) \times} \\
\sqrt{\left(\frac{v_{\mathrm{t} x}}{\xi+\frac{D}{C \xi}}+\frac{v_{\mathrm{r} x}}{\xi-\frac{D}{C \xi}}\right)^{2}+\left(\frac{v_{\mathrm{t} y}}{\xi+\frac{D}{C \xi}}+\frac{v_{\mathrm{r} y}}{\xi-\frac{D}{C \xi}}\right)^{2}}
\end{gathered}
$$

which bounds the resulting Doppler pdf. The latter is influenced by the movement of the transmitter and receiver in directions orthogonal to the $z$-axis.

Note that the resulting pdf is independent of the length of the intersection circle similar to the general case, which means same distance ratios between scattering plane and TXRX distance result in the same pdf. Moreover, we see from (42) that the resulting delay-dependent Doppler pdf coincides with a (shifted) Jakes spectrum. This is expected for uniformly distributed scatterers on a circle. The shift comes from the movement towards or away from the scattering plane along the $z$-axis. In contrast, the width of the spectrum is determined by the velocity components in $x$ - and $y$-directions. For large delays, i.e., when $\xi \rightarrow \infty, \lim _{\xi \rightarrow \infty} f_{\mathrm{o}}(\xi)=0 \mathrm{~Hz}$ and the pdf $p\left(t, q_{\xi}(\eta, \vartheta) ; f_{\mathrm{d}} \mid \xi\right)$ becomes centered at $0 \mathrm{~Hz}$. Thus, in the limit, we obtain classical "non-shifted" Jakes spectra, and the velocity components in $x$ - and $y$-directions alone impact the width of Doppler pdf.

\section{Joint Delay Doppler PDF}

Our goal in this section is to compute the joint delay Doppler pdf. The approach is similar to the delay-dependent Doppler pdf, but now includes the variable $\xi$, which thus creates a two-dimensional density. Again, we will consider the general case and the complementary case. The former is most easily derived from the last line in (20), while the latter corresponds to the first line in (20) with $A=B=0$.

After replacing the wedge products of the Cartesian differentials with the differentials in PSCs according to (16), the area of the scattering ellipse can be expressed in PSCs. For 
the general case the differential area of an arbitrary plane is calculated as

$$
r_{i} \mathrm{~d} S_{i}=\frac{l^{2} \sqrt{A^{2}+B^{2}+C^{2}}\left(\xi^{2}-\eta^{2}\right)}{\sqrt{\left(\xi^{2}-1\right)\left(1-\eta^{2}\right)\left(A^{2}+B^{2}\right)-(D-C \xi \eta)^{2}}} \mathrm{~d} \eta \mathrm{d} \xi
$$

where $i \in\{1,2,3\}$ corresponds to the three integrals in (20). It determines the differential area needed to compute the total area of the ellipse defined by the delay ellipsoid and the scattering plane. Here, the wedge product is replaced by a normal product, since we made sure that the limits of the integrals together with the integrand produce a positive area.

For the complementary case the differential area of a scattering plane orthogonal to the $z$-axis in PSCs results in

$$
r_{1} \mathrm{~d} S_{1}=l^{2}\left(\xi-\frac{D^{2}}{C^{2} \xi^{3}}\right) \mathrm{d} \vartheta \mathrm{d} \xi
$$

which corresponds to the first line in (20) with $A=B=0$ and thus $r_{1}=1$. The integral over the differential area thus calculates the area of a circle, which is created by intersecting the scattering plane and the delay ellipsoid, since the scattering plane is orthogonal to the $z$-axis.

\section{A. Path Loss Inspired Weighting Function}

To introduce the impact of the path loss induced by scatterers we make use of the classical bistatic radar power equation under the assumption that the radar cross section of the scatterers is constant in both space and time. Under this assumption the received power $P$ is proportional to $P \propto\left(d_{\mathrm{t}}^{2} d_{\mathrm{r}}^{2}\right)^{-1}$ inferred by the radar equation, where $d_{\mathrm{t}}$ and $d_{\mathrm{r}}$ are given in (2). This assumption is only valid for scattered signals with $\xi>\xi_{\text {sr }}$ [44]-[46]. Thus, we can make the joint delay Doppler pdf proportional to the time-variant scattering function.

By inserting (2) into $P \propto\left(d_{\mathrm{t}}^{2} d_{\mathrm{r}}^{2}\right)^{-1}$ and simplifying the result, we compute a weighting function as

$$
w(\xi, \eta)=\frac{1}{\left(\xi^{2}-\eta^{2}\right)^{2}} .
$$

Note that the weighting function in our case is only a function of $\xi$ and $\eta$. Contours of constant $w(\xi, \eta)$ are known from the radar literature as Cassini ovals [47]. These are locations where the product $d_{\mathrm{t}}^{2} d_{\mathrm{r}}^{2}$ is constant. An important consequence of this is that the Cassini ovals generally do not coincide with the intersection ellipses, since their product of distances rather than the sum is constant. This implies that scatterers lying on intersection ellipses are in general weighted differently, which has a direct impact for the calculation of the joint pdf as we show subsequently.

A special case occurs for the complementary case, i.e., when the scattering plane is orthogonal to the major axis of the ellipsoid. For this particular orientation of the scattering plane the intersection area is a circle. Thus, the weighting function $w(\xi, \eta)$ only depends on the $\xi$-coordinate, since the intersection is characterized by a constant $\eta$-coordinate. The Cassini oval of constant path loss is also a circle when the oval is intersected orthogonally by the scattering plane. It coincides with the intersection circle created by the delay ellipsoid. This permits a factorization of the joint delay Doppler pdf into the product of the marginals, i.e., the delay-dependent Doppler pdf computed earlier and the path loss pdf. For a general orientation of the scattering plane such a factorization is only possible with simple factors for $\xi \rightarrow \infty$.

\section{B. Calculating the Normalization Coefficient}

By building upon the results for the delay-dependent Doppler pdf, we derive the joint delay Doppler pdf.

For the general case the situation is a bit more involved. We first compute the weighted area $\mathcal{Y}_{1}$ of the intersection $q(\xi, \eta)=0$. Note that in this case the density of the scatterers $\mathbf{s}$ is described by the $(\xi, \eta)$-coordinates. Using $r_{i} \mathrm{~d} S_{i}$ from (45) and the weighting function in (47), we obtain

$$
\int_{\xi_{\min }}^{\mathcal{Y}_{1}} 2 \int_{\eta_{\min }(\xi)}^{\xi_{\max }} \frac{w(\xi, \eta) l^{2} \sqrt{A^{2}+B^{2}+C^{2}}\left(\xi^{2}-\eta^{2}\right) \mathrm{d} \eta \mathrm{d} \xi}{\sqrt{\left(\xi^{2}-1\right)\left(1-\eta^{2}\right)\left(A^{2}+B^{2}\right)-(D-C \xi \eta)^{2}}},
$$

where the factor of two is needed to account for both of the half ellipses. The Jacobian for the mapping $(\xi, \eta) \mapsto\left(\xi, f_{\mathrm{d}}\right)$ is computed as

$$
\mathbf{J}_{\mathbf{s}}=\left(\begin{array}{cc}
\frac{\partial \xi}{\partial \xi} & \frac{\partial \xi}{\partial \eta} \\
\frac{\partial f_{\mathrm{d}}}{\partial \xi} & \frac{\partial f_{\mathrm{d}}}{\partial \eta}
\end{array}\right), \quad\left|\mathbf{J}_{\mathbf{s}}{ }^{-1}\right|=\frac{\partial \eta}{\partial f_{\mathrm{d}}} .
$$

The challenge in computing the joint pdf is in recognizing that for the mapping $\eta \mapsto f_{\mathrm{d}}$ we need to use the algebraic curve $\mathcal{C}_{42}^{\star}$, which results in up to $|\mathcal{H}| \leq 4$ solutions for $\eta_{j}^{\star} \mapsto f_{\mathrm{d}}$, $j=1, \ldots,|\mathcal{H}|$. Each solution is only valid in a certain interval. Then, the joint pdf is computed as

$$
\begin{aligned}
& p\left(t, q(\xi, \eta) ; \xi, f_{\mathrm{d}}\right)=\sum_{j=1}^{|\mathcal{H}|} \frac{1}{\mathcal{Y}_{1}} \times \\
& \frac{l^{2} \sqrt{A^{2}+B^{2}+C^{2}}\left(\xi^{2}-\left(\eta_{j}^{\star}\right)^{2}\right)^{-1}}{\sqrt{\left(\xi^{2}-1\right)\left(1-\left(\eta_{j}^{\star}\right)^{2}\right)\left(A^{2}+B^{2}\right)-\left(D-C \xi \eta_{j}^{\star}\right)^{2}}}\left|\frac{\partial \eta_{j}^{\star}}{\partial f_{\mathrm{d}}}\right| .
\end{aligned}
$$

By analyzing (50) in more detail, we see that the joint delay Doppler pdf does not factor into $p\left(t, q_{\xi}(\eta) ; f_{\mathrm{d}} \mid \xi\right)$ given by (30) and a delay pdf $p(t, q(\xi, \eta) ; \xi)$. The reason for this is the fact that for generally oriented scattering planes the scatterers lying along the intersection ellipses do not correspond to the Cassini ovals of constant path loss as explained above.

The joint delay Doppler pdf for the complementary case is calculated as follows. Consider the intersection $q(\xi, \vartheta)=0$ with the weighted area $\mathcal{Y}_{2}$ of the plane intersection ellipse. With the differential area $r_{1} \mathrm{~d} S_{1}$ from (46) and the weighting function $w(\xi, \eta)$ in (47) as

$$
\mathcal{Y}_{2}=\iint_{q(\xi, \eta, \vartheta)=0} w\left(\xi, \frac{D}{C \xi}\right) \mathrm{d} S_{1}=\int_{\xi \min }^{\xi_{\max }} \int_{0}^{2 \pi} \frac{l^{2}\left(\xi-\frac{D^{2}}{C^{2} \xi^{3}}\right)}{\left(\xi^{2}-\left(\frac{D}{C \xi}\right)^{2}\right)^{2}} \mathrm{~d} \vartheta \mathrm{d} \xi
$$


with $\xi_{\max }>\xi_{\min }>\xi_{\mathrm{sr}}$. The joint delay Doppler pdf for the complementary case is computed using the transformation in (9) and the Jacobian

$$
\mathbf{J}_{\mathbf{s}}=\left(\begin{array}{cc}
\frac{\partial \xi}{\partial \xi} & \frac{\partial \xi}{\partial \vartheta} \\
\frac{\partial f_{\mathrm{d}}}{\partial \xi} & \frac{\partial f_{\mathrm{d}}}{\partial \vartheta}
\end{array}\right), \quad\left|\mathbf{J}_{\mathbf{s}}{ }^{-1}\right|=\frac{\partial \vartheta}{\partial f_{\mathrm{d}}} .
$$

By following the same steps as for the delay-dependent Doppler pdf in (42), we obtain

$$
\begin{aligned}
& p\left(t, q(\xi, \vartheta) ; \xi, f_{\mathrm{d}}\right)= \\
& \frac{2 l^{2}\left(\xi-\frac{D^{2}}{C^{2} \xi^{3}}\right)}{\mathcal{Y}_{2}\left(\xi^{2}-\left(\frac{D}{C \xi}\right)^{2}\right)^{2}\left|f_{\lim }\right| \sqrt{1-\left(\frac{f_{\mathrm{d}}-f_{\mathrm{o}}}{f_{\lim }}\right)^{2}}} .
\end{aligned}
$$

Let us stress that the mapping $f_{\mathrm{d}} \mapsto \vartheta$ is ambiguous due to the arccos function, as explained in Sec. II. Thus, similar to the delay-dependent Doppler pdf, we need to account for this ambiguity with a factor of two, which eventually leads to (53). By carefully studying (53) we recognize that in the complimentary case the joint pdf factors into the delay-dependent Doppler pdf $p\left(t, q_{\xi}(\vartheta) ; f_{\mathrm{d}} \mid \xi\right)$ given by (42) and a delay pdf $p(t, q(\xi, \vartheta) ; \xi)$ - a model of the classical power delay profile. Thus, in the complementary case the joint pdf can be expressed by $p\left(t, q(\xi, \vartheta) ; \xi, f_{\mathrm{d}}\right)=p\left(t, q_{\xi}(\vartheta) ; f_{\mathrm{d}} \mid \xi\right) p(t, q(\xi, \vartheta) ; \xi)$.

\section{RESULTS}

In the following, we present generalizations of the closed form solutions for three special components discussed in [17]: the LOS component, the SR component, and the diffuse scattering components for infinite delays. According to Bello [48], the surface scatter channel can be modeled by

$$
h(t)=h_{\mathrm{los}}(t)+h_{\mathrm{sr}}(t)+h_{\mathrm{sc}}(t)+n(t) .
$$

This can be seen as combination of the LOS channel, the SR channel, the scattering channel, and an additive noise term $n(t)$. Based on this model, we can place our results in the same context. For any time instance $t$, the non-stationary joint delay Doppler pdf represents the contribution of the scattering component $h_{\mathrm{sc}}(t)$ of the M2M channel. The other two components $h_{\mathrm{los}}(t)$ and $h_{\mathrm{sr}}(t)$ are typically modeled as deterministic components. For LOS and SR signals, a free-space path loss model should be used. For the specular reflection signal the reflection coefficient as additional attenuation has to be taken into account. In the following, we show that the equations derived here generalize results for the above mentioned special cases known from the literature. To this end, we use the delaydependent Doppler pdfs and its inverse Fourier transform, the delay-dependent characteristic function, to model the LOS component, the SR component, and the scattering components of the time-variant channel $h(t)$, since the joint pdf would be zero due to the path loss attenuation for $\xi \rightarrow \infty$.

\section{A. LOS Component}

The results for the LOS component can be obtained for $\xi=1$, yet care has to be taken in deriving the delay-dependent pdf. Specifically, for $\xi=1$ the scattering plane should not intersect the LOS, because it would block the signal path in this case, as we have mentioned earlier. Thus, for $\xi=1$ the Doppler frequency in (27) becomes independent of the scattering plane parameters and the spatial coordinates. As such, the LOS component consists of a single frequency and the Doppler pdf becomes a Dirac distribution centered at this frequency. The characteristic function $\Phi$, its corresponding delay-dependent Doppler pdf $p$, the mean Doppler $\mu$, and the Doppler spread $\sigma$ are expressed as

$$
\begin{aligned}
& \Phi\left(t, q_{\xi}(\eta, \vartheta) ; u \mid \xi\right)=\exp \left(\mathrm{j} 2 \pi u f_{\mathrm{los}}(t)\right), \\
& p\left(t, q_{\xi}(\eta, \vartheta) ; f_{\mathrm{d}} \mid \xi\right)=\delta\left(f_{\mathrm{d}}-f_{\mathrm{los}}(t)\right), \\
& \mu\left(t, q_{\xi}(\eta, \vartheta)\right)=\frac{v_{\mathrm{t} z}-v_{\mathrm{r} z}}{c} f_{\mathrm{c}}=f_{\mathrm{los}}(t), \\
& \sigma\left(t, q_{\xi}(\eta, \vartheta)\right)=0 .
\end{aligned}
$$

\section{B. SR Component}

The specular reflection from the scattering plane occurs if $\xi=\xi_{\text {sr }}$. The results for the specular component can be obtained as a limiting case of the general expressions that were derived in this paper. Specifically, evaluating (30) and (42) for $\xi \rightarrow \xi_{\text {sr }}$, we obtain for the delay-dependent Doppler pdf $p$. For the characteristic function $\Phi$, the mean Doppler $\mu$ and the Doppler spread $\sigma$, it follows

$$
\begin{aligned}
& \Phi\left(t, q_{\xi}(\eta, \vartheta) ; u \mid \xi\right)=\exp \left(\mathrm{j} 2 \pi u f_{\mathrm{sr}}(t)\right), \\
& p\left(t, q_{\xi}(\eta, \vartheta) ; f_{\mathrm{d}} \mid \xi\right)=\delta\left(f_{\mathrm{d}}-f_{\mathrm{sr}}(t)\right), \\
& \mu\left(t, q_{\xi}(\eta, \vartheta)\right)=f_{\mathrm{sr}}(t), \quad \sigma\left(t, q_{\xi}(\eta, \vartheta)\right)=0,
\end{aligned}
$$

with

$$
\begin{aligned}
\xi_{\mathrm{sr}} & =\max \left(\sqrt{\frac{A^{2}+B^{2}+D^{2}}{A^{2}+B^{2}+C^{2}}}, 1\right), \\
\eta_{\mathrm{sr}} & =\frac{D C \xi_{\mathrm{sr}}}{A^{2} \xi_{\mathrm{sr}}^{2}+B^{2} \xi_{\mathrm{sr}}^{2}+C^{2} \xi_{\mathrm{sr}}^{2}-A^{2}-B^{2}}, \\
f_{\mathrm{sr}}(t) & = \begin{cases}f_{\mathrm{d}, i}^{\star}\left(t, \xi_{\mathrm{sr}}, \eta_{\mathrm{sr}}\right) & \text { general case, } \\
f_{\mathrm{o}}\left(t, \xi_{\mathrm{sr}}\right) & \text { complementary case. }\end{cases}
\end{aligned}
$$

Note that if $\xi_{\mathrm{sr}}=1$, we obtain the LOS component with the scattering plane placed at the location of the TX or the RX; LOS and SR component coincide in this case. For $\xi_{\text {sr }}>1$ the SR component and LOS are distinct.

\section{Scattering Components}

In the limit for $\xi \rightarrow \infty$ and using (30) and (42) for the delaydependent Doppler pdf, we obtain the following stochastic functions for the scattering components

$$
\begin{aligned}
& \lim _{\xi \rightarrow \infty} \Phi\left(t, q_{\xi}(\eta, \vartheta) ; u \mid \xi\right)=J_{0}\left(2 \pi u \frac{\left\|\mathbf{v}_{\mathrm{t} \| \mathrm{E}}+\mathbf{v}_{\mathrm{r} \| \mathrm{E}}\right\|}{c} f_{\mathrm{c}}\right) \\
& \lim _{\xi \rightarrow \infty} p\left(t, q_{\xi}(\eta, \vartheta) ; f_{\mathrm{d}} \mid \xi\right)=\frac{1}{\pi\left|f_{1,2}\right| \sqrt{1-\left(\frac{f_{\mathrm{d}}}{f_{1,2}(t)}\right)^{2}}},
\end{aligned}
$$

$$
\begin{aligned}
& \lim _{\xi \rightarrow \infty} \mu\left(t, q_{\xi}(\eta, \vartheta)\right)=0, \\
& \lim _{\xi \rightarrow \infty} \sigma\left(t, q_{\xi}(\eta, \vartheta)\right)=\frac{\left\|\mathbf{v}_{\mathrm{t} \| \mathrm{E}}+\mathbf{v}_{\mathbf{r} \| \mathrm{E}}\right\|}{\sqrt{2} c} f_{\mathrm{c}}=\frac{f_{1,2}(t)}{\sqrt{2}},
\end{aligned}
$$




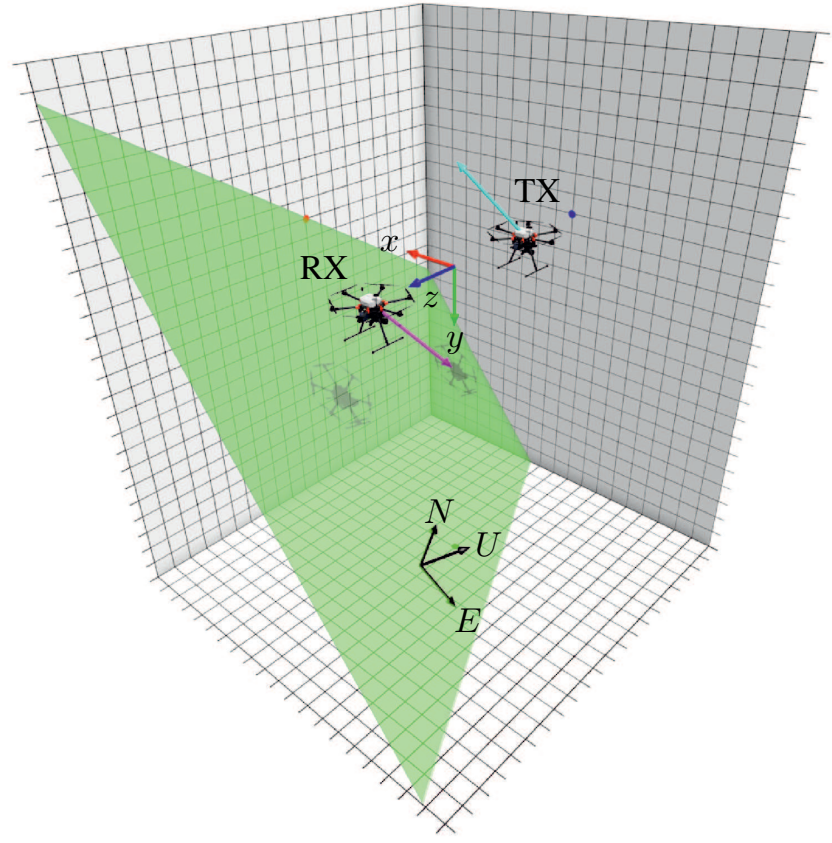

Fig. 4. Drone-to drone scenario with $A_{0}=1, B_{0}=0.8, C_{0}=0.5$, $D_{0}=2$, and $l_{0}=50 \mathrm{~m}$ with velocity vectors $\mathbf{v}_{\mathrm{t} 0}=[25,-35,20]^{\mathrm{T}} \mathrm{km} / \mathrm{h}$ and $\mathbf{v}_{\mathrm{r} 0}=[-30,25,-15]^{\mathrm{T}} \mathrm{km} / \mathrm{h}$. The TX and RX location is marked by the drones. The origins of the moving local Cartesian coordinate system, which is located between the drones, and the fixed ENU coordinate system, which is located in the scattering plane, is shown.

with parallel velocity vectors and limiting frequencies

$$
\begin{aligned}
& \mathbf{v}_{\mathrm{t} \| \mathrm{E}}=\frac{\mathbf{n}_{\mathrm{E}} \times\left(\mathbf{v}_{\mathrm{t}} \times \mathbf{n}_{\mathrm{E}}\right)}{\left\|\mathbf{n}_{\mathrm{E}}\right\|^{2}}=\mathbf{v}_{\mathrm{t}}-\frac{\left(\mathbf{v}_{\mathrm{t}} \cdot \mathbf{n}_{\mathrm{E}}\right) \mathbf{n}_{\mathrm{E}}}{\left\|\mathbf{n}_{\mathrm{E}}\right\|^{2}}, \\
& \mathbf{v}_{\mathrm{r} \| \mathrm{E}}=\frac{\mathbf{n}_{\mathrm{E}} \times\left(\mathbf{v}_{\mathrm{r}} \times \mathbf{n}_{\mathrm{E}}\right)}{\left\|\mathbf{n}_{\mathrm{E}}\right\|^{2}}=\mathbf{v}_{\mathrm{r}}-\frac{\left(\mathbf{v}_{\mathrm{r}} \cdot \mathbf{n}_{\mathrm{E}}\right) \mathbf{n}_{\mathrm{E}}}{\left\|\mathbf{n}_{\mathrm{E}}\right\|^{2}}, \\
& \lim _{\xi \rightarrow \infty} f_{1,2}(t)= \pm \frac{\left\|\mathbf{v}_{\mathrm{t} \| \mathrm{E}}+\mathbf{v}_{\mathrm{r} \| \mathrm{E}}\right\|}{c} f_{\mathrm{c}} .
\end{aligned}
$$

Here the velocity vectors $\mathbf{v}_{\mathrm{t} \| \mathrm{E}}=\left[v_{\mathrm{t} x \| \mathrm{E}}, v_{\mathrm{t} y \| \mathrm{E}}, v_{\mathrm{t} z \| \mathrm{E}}\right]^{\mathrm{T}}$ and $\mathbf{v}_{\mathrm{r} \| \mathrm{E}}=\left[v_{\mathrm{r} x \| \mathrm{E}}, v_{\mathrm{r} y \| \mathrm{E}}, v_{\mathrm{r} z \| \mathrm{E}}\right]^{\mathrm{T}}$ are parallel to the scattering plane. The limiting frequencies $f_{1,2}(t)$ are given by the solution $\eta_{1,2}= \pm\left(v_{\mathrm{t} z \| \mathrm{E}}+v_{\mathrm{r} z \| \mathrm{E}}\right) /\left(\left\|\mathbf{v}_{\mathrm{t} \| \mathrm{E}}+\mathbf{v}_{\mathrm{r} \| \mathrm{E}}\right\|\right)$ of the polynomial in (31). This result matches the classical Jakes result. The width of the spectrum, however, is determined by the velocity vector components of $\mathrm{TX}$ and $\mathrm{RX}$, which are parallel to the scattering plane. The reason for this is that for large $\xi$ the ellipsoid becomes a sphere. The intersection with the scattering plane results in a scattering circle on which the scatterers are uniformly distributed.

\section{Numerical Results}

In this section, we evaluate the presented model for an exemplary scenario. In order to take current technology into account and to show the full 3D capability of the model, we selected a time-variant drone-to-drone scenario. More examples on the capability of our model to represent timevariant scenarios can be found in [49]. The carrier frequency for the scenario is $f_{\mathrm{c}}=2.4 \mathrm{GHz}$ [50], which is a typical frequency for drone-to-drone communication. We evaluate the scenario for $t_{0}=0 \mathrm{~s}, t_{1}=1 \mathrm{~s}$, and $t_{2}=2 \mathrm{~s}$. The initial distance between the flying TX and RX is $2 l_{0}=100 \mathrm{~m}$. The scattering plane is below the drones and inclined. It is given for $t_{0}=0 \mathrm{~s}$ by $A_{0} x+B_{0} y+C_{0} z=l_{0} D_{0}$, with $A_{0}=1, B_{0}=0.8, C_{0}=0.5$ and $D_{0}=2$. The velocity vectors are mainly parallel to the scattering plane and given by $\mathbf{v}_{\mathrm{t} 0}=[25,-35,20]^{\mathrm{T}} \mathrm{km} / \mathrm{h}$ and $\mathbf{v}_{\mathrm{r} 0}=[-30,25,-15]^{\mathrm{T}} \mathrm{km} / \mathrm{h}$. This means the drones are in a fly-by scenario as shown in Fig. 4. The parameters for other two time steps $t_{1}$ and $t_{2}$ are obtained by rotating the local Cartesian coordinate system along the $x$ - and $y$-axis according to the $x$ - and $y$-components of the velocity vectors of TX and RX. The velocity vectors in $z$-direction only cause a stretching of the $z$-axis, but no rotation.

The joint delay Doppler pdfs for $t_{0}=0 \mathrm{~s}$ are shown in Fig. 5. As expected the probability decreases with increasing delay, since we included the path loss in our model. Furthermore, the four poles of the joint pdf can be recognized. The poles are calculated by (31) and are shown as black lines in Fig. 5. The joint pdf matches perfectly with the limiting frequencies, which shows the validity of the sixth order

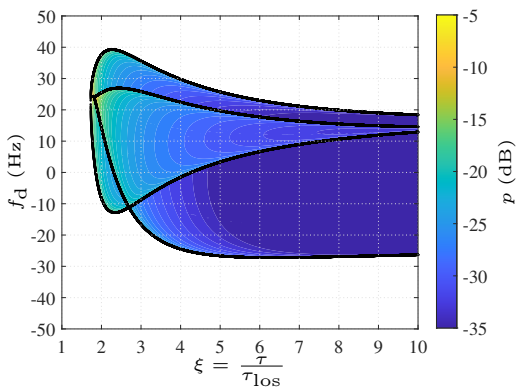

(a) For $t_{0}=0 \mathrm{~s}$ with parameters $A_{0}=1$, $B_{0}=0.8, C_{0}=0.5, D_{0}=2, l_{0}=$ $50 \mathrm{~m}, \mathbf{v}_{\mathrm{t} 0}=[25,-35,20]^{\mathrm{T}} \mathrm{km} / \mathrm{h}$ and $\mathbf{v}_{\mathrm{r} 0}=$ $[-30,25,-15]^{\mathrm{T}} \mathrm{km} / \mathrm{h}$.

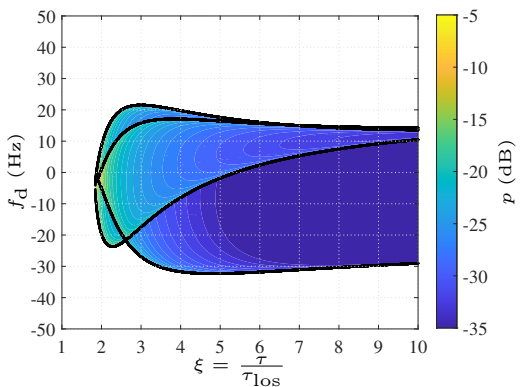

(b) For $t_{1}=1 \mathrm{~s}$ with parameters $A_{1}=1$, $B_{1}=0.64, C_{1}=0.43, D_{1}=2.00, l_{1}=$ $46.53 \mathrm{~m}, \mathbf{v}_{\mathrm{t} 1}=[26.85,-38.05,9.03]^{\mathrm{T}} \mathrm{km} / \mathrm{h}$ and $\mathbf{v}_{\mathrm{r} 1}=[-31.27,27.31,-5.15]^{\mathrm{T}} \mathrm{km} / \mathrm{h}$.

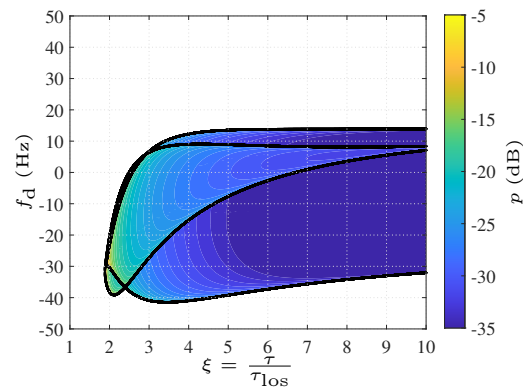

(c) For $t_{2}=2 \mathrm{~s}$ with parameters $A_{2}=1, B_{2}=0.46, C_{2}=0.33$, $D_{2}=1.86, \quad l_{2}=46.19 \mathrm{~m}$ $\mathbf{v}_{\mathrm{t} 2}=[25.28,-39.99,-3.46]^{\mathrm{T}} \mathrm{km} / \mathrm{h}$ and $\mathbf{v}_{\mathrm{r} 2}=[-29.73,28.84,5.86]^{\mathrm{T}} \mathrm{km} / \mathrm{h}$.

Fig. 5. Time-variant joint delay Doppler pdf $p\left(t, q(\xi, \eta) ; \xi, f_{\mathrm{d}}\right)$ according to (50) and limiting frequencies (black lines) according to (31). 

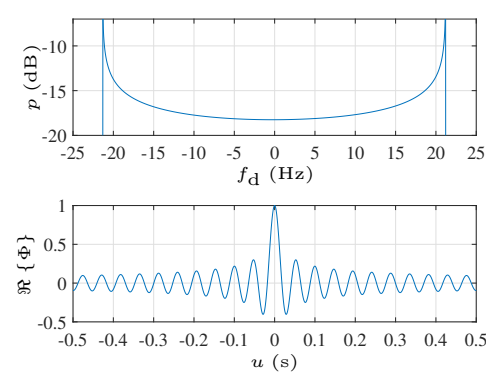

(a) For $t_{0}=0 \mathrm{~s}$ with velocity vectors $\mathbf{v}_{\mathrm{t} 0 \| \mathrm{E}}=[21.30,-37.96,18.15]^{\mathrm{T}} \mathrm{km} / \mathrm{h}$ and $\mathbf{v}_{\mathrm{r} 0 \| \mathrm{E}}=[-20.74,32.41,-10.37]^{\mathrm{T}} \mathrm{km} / \mathrm{h}$. The limiting frequency according to (60) becomes $f_{1,2}\left(t_{0}\right)= \pm 21.28 \mathrm{~Hz}$ and the first zero crossing of the Bessel function according to (58) is at $u=2.405 /\left(2 \pi f_{1,2}\left(t_{0}\right)= \pm 0.018 \mathrm{~s}\right.$.
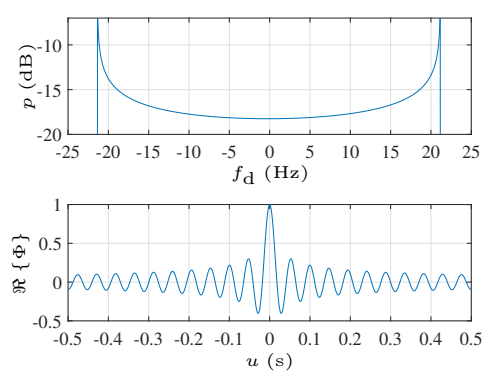

(b) For $t_{1}=1 \mathrm{~s}$ with velocity vectors $\mathbf{v}_{\mathrm{t} 1 \| \mathrm{E}}=[22.81,-40.63,7.31]^{\mathrm{T}} \mathrm{km} / \mathrm{h}$ and $\mathbf{v}_{\mathrm{r} 1 \| \mathrm{E}}=[-21.17,33.75,-0.85]^{\mathrm{T}} \mathrm{km} / \mathrm{h}$. The limiting frequency according to (60) becomes $f_{1,2}\left(t_{1}\right)= \pm 21.28 \mathrm{~Hz}$ and the first zero crossing of the Bessel function according to (58) is at $u=2.405 /\left(2 \pi f_{1,2}\left(t_{1}\right)= \pm 0.018 \mathrm{~s}\right.$.
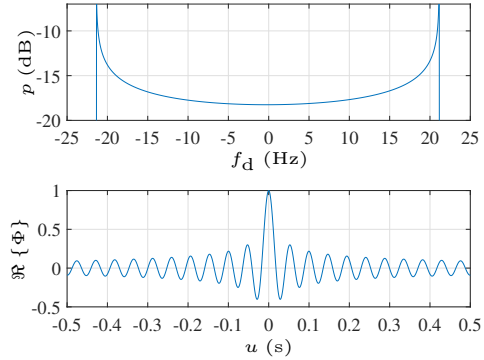

(c) For $t_{2}=2 \mathrm{~s}$ with velocity vectors $\mathbf{v}_{\mathrm{t} 2 \| \mathrm{E}}=[20.84,-42.02,-4.92]^{\mathrm{T}} \mathrm{km} / \mathrm{h}$ and $\mathbf{v}_{\mathrm{r} 2 \| \mathrm{E}}=[-18.64,33.91,9.51]^{\mathrm{T}} \mathrm{km} / \mathrm{h}$. The limiting frequency according to (60) becomes $f_{1,2}\left(t_{2}\right)= \pm 21.28 \mathrm{~Hz}$ and the first zero crossing of the Bessel function according to (58) is at $u=2.405 /\left(2 \pi f_{1,2}\left(t_{2}\right)= \pm 0.018 \mathrm{~s}\right.$.

Fig. 6. Delay-dependent Doppler pdf $p\left(t, q_{\xi}(\eta) ; f_{\mathrm{d}} \mid \xi=1000\right)$ according to (30) and real part of the delay-dependent characteristic function $\Re\left\{\Phi\left(t, q_{\xi}(\eta, \vartheta) ; u \mid \xi=1000\right)\right\}$.

polynomial. The specular reflection occurs according to (57) at $\xi_{\text {sr }}=1.7275$ with a Doppler frequency of $f_{\text {sr }}\left(t_{0}\right)=22.39 \mathrm{~Hz}$ in Fig. 5a, which is indicated by the gap of the black lines. The delay-dependent Doppler pdfs for $\xi=1000$ are shown in the upper part of Fig. 6 for the three time instances. Since this approximates the scattering components for $\xi \rightarrow \infty$, we obtain a Jakes Doppler spectrum with the same limiting frequencies for all three cases. The comparison between the limits of the delay-dependent pdfs in Fig. 6 and the closedform solution $f_{1,2}(t)= \pm 21.3 \mathrm{~Hz}$ from (60) shows perfect agreement. In the lower part of Fig. 6, the real part of the time-variant characteristic functions are shown. We obtain a zeroth-order Bessel function of the first kind, which is the inverse Fourier transform of the Doppler spectrum in the upper part, for all three time instances. The first zero crossing of the characteristic functions in Fig. 6 occurs at $u=2.405 /\left(2 \pi f_{1,2}(t)\right)= \pm 0.018 \mathrm{~s}$ with $f_{1,2}(t)= \pm 21.3 \mathrm{~Hz}$, which perfectly matches the theory.

The fly-by scenario causes the joint delay Doppler pdf in Fig. 5a, 5b, and Fig. 5c to become time-variant. At the beginning the drones are flying towards each other, which can be seen by the positive Doppler frequencies in Fig. 5a. The Doppler frequencies are the almost centered around $0 \mathrm{~Hz}$ in Fig. $5 b$ and then turn negative in Fig. 5c, since now they fly away from each other. The change of the scattering plane parameters and the velocity vectors of TX and RX is shown in the captions of Figs. 5a, 5b, and 5c. Note that the scattering plane parameters and velocity vectors remain constant in the ENU coordinate system. This is the reason, why for large delays $\xi$ the geometric relationship between velocity vectors and scattering plane does not change. The delay-dependent Doppler pdf for large delays would only change, if the velocity vectors in the ENU coordinate system become time-variant. Therefore, the delay-dependent Doppler pdfs in Figs. $6 a, 6 b$, and $6 c$ remain constant, although the parameters and parallel velocity vectors in the local coordinate system change. The change of parameter values is shown in the captions of Figs. $6 a, 6 b$, and $6 c$.

\section{CONCLUSION}

In this paper, we derived a general, time-variant, threedimensional, single-bounce scattering model for mobile-tomobile channels. It is based on prolate spheroidal coordinates, the theory of algebraic curves, and differential forms. The prolate spheroidal coordinate system allows a more natural description of the mobile-to-mobile channel and thus enabled us to carry out a geometric algebraic analysis of the Doppler frequency. The theory of algebraic curves was introduced during the calculation of the Doppler probability density function in order to remove the ambiguity in the description. Thus, we could determine the functional relationship between the spatial variables and the delay and Doppler frequency variables, which is the prerequisite to derive the delay-dependent and the joint delay Doppler probability density functions. Finally, differential forms were needed to normalize the obtained probability density functions on the basis of the length or equivalent area.

The introduced theoretical framework enabled us to derive the delay-dependent Doppler pdf and the joint delay Doppler pdf for general time-variant, mobile-to-mobile channels. Hereby, the joint delay Doppler pdf was calculated using a bistatic radar inspired path loss model. Additionally, we determined the limiting frequencies of the mentioned probability density functions as horizontal tangents of the Doppler algebraic curve. The theoretical results fully agree with our previous models and measurement data. Thus, the theory can be applied to arbitrary mobile-to-mobile channels in order to describe and simulate them both realistically and efficiently.

\section{ACKNOWLEDGMENT}

The authors would like to thank Thomas Wiedemann for the help with the graphics in the manuscript. Furthermore, we would like to acknowledge the numerous fruitful discussions in COST Action CA15104 IRACON. 


\section{REFERENCES}

[1] R. H. Clarke, "A statistical theory of mobile-radio reception," Bell Syst. Tech. J., vol. 47, no. 6, pp. 957-1000, Jul./Aug. 1968.

[2] P. A. Bello, "Characterization of randomly time-variant linear channels," IEEE Trans. Commun., vol. 11, no. 4, pp. 360-393, Dec. 1963.

[3] P. Hoeher, "A statistical discrete-time model for the WSSUS multipath channel," IEEE Trans. Veh. Technol., vol. 41, no. 4, pp. 461-468, Nov. 1992.

[4] W. C. Jakes, Microwave Mobile Communications, ser. IEEE Press Classic Reissue. New York, USA: Wiley, 1994.

[5] A. S. Akki and F. Haber, "A statistical model of mobile-to-mobile land communication channel," IEEE Trans. Veh. Technol., vol. 35, no. 1, pp. 2-7, Feb. 1986

[6] F. Vatalaro and A. Forcella, "Doppler spectrum in mobile-to-mobile communications in the presence of three-dimensional multipath scattering," IEEE Trans. Veh. Technol., vol. 46, no. 1, pp. 213-219, Feb. 1997.

[7] C. S. Patel, G. L. Stüber, and T. G. Pratt, "Simulation of Rayleigh-faded mobile-to-mobile communication channels," IEEE Trans. Commun., vol. 53, no. 11, pp. 1876-1884, Nov. 2005.

[8] Z. Tang and A. S. Mohan, "A correlated indoor MIMO channel model," in Can. Conf. Elect. Comput. Eng., Montreal, QC, Canada, May 2003, pp. 1889-1892.

[9] A. G. Zajić, G. L. Stüber, T. G. Pratt, and S. T. Nguyen, "Wideband MIMO mobile-to-mobile channels: Geometry-based statistical modeling with experimental verification," IEEE Trans. Veh. Technol., vol. 58, no. 2 , pp. 517-534, Feb. 2009.

[10] M. Pätzold, B. O. Hogstad, N. Youssef, and D. Kim, "A MIMO mobileto-mobile channel model: Part I - the reference model," in Proc. IEEE 16th Int. Symp. Pers., Indoor and Mobile Radio Commun., Berlin, Germany, Sep. 2005, pp. 573-578.

[11] A. Abdi and M. Kaveh, "A space-time correlation model for multielement antenna systems in mobile fading channels," IEEE J. Sel. Areas Commun., vol. 20, no. 3, pp. 550-560, Apr. 2002.

[12] M. Pätzold, B. O. Hogstad, and N. Youssef, "Modeling, analysis, and simulation of MIMO mobile-to-mobile fading channels," IEEE Trans. Wireless Commun., vol. 7, no. 2, pp. 510-520, Feb. 2008.

[13] A. G. Zajić and G. L. Stüber, "Three-dimensional modeling, simulation, and capacity analysis of space-time correlated mobile-to-mobile channels," IEEE Trans. Veh. Technol., vol. 57, no. 4, pp. 2042-2054, Jul. 2008.

[14] — "Three-dimensional modeling and simulation of wideband MIMO mobile-to-mobile channels," IEEE Trans. Wireless Commun., vol. 8, no. 3, pp. 1260-1275, Mar. 2009.

[15] A. Paier, T. Zemen, L. Bernadó, G. Matz, J. Karedal, N. Czink, C. Dumard, F. Tufvesson, A. F. Molisch, and C. F. Mecklenbräuker, "Non-WSSUS vehicular channel characterization in highway and urban scenarios at $5.2 \mathrm{GHz}$ using the local scattering function," in Int. ITG Workshop on Smart Antennas (WSA), Darmstadt, Germany, Feb. 2008, pp. 9-15.

[16] L. Bernadó, "Non-stationarity in vehicular wireless channels," Ph.D. dissertation, Technische Universität Wien, Vienna, Austria, Apr. 2012.

[17] M. Walter, D. Shutin, D. W. Matolak, N. Schneckenburger, T. Wiedemann, and A. Dammann, "Analysis of non-stationary 3D air-to-air channels using the theory of algebraic curves," IEEE Trans. Wireless Commun., vol. 18, no. 8, pp. 3767-3780, Aug. 2019.

[18] G. Matz, "On non-WSSUS wireless fading channels," IEEE Trans. Wireless Commun., vol. 4, no. 5, pp. 2465-2478, Sep. 2005.

[19] J. Karedal, F. Tufvesson, N. Czink, A. Paier, C. Dumard, T. Zemen, C. F. Mecklenbräuker, and A. F. Molisch, "A geometry-based stochastic MIMO model for vehicle-to-vehicle communications," IEEE Trans. Wireless Commun., vol. 8, no. 7, pp. 3646-3657, Jul. 2009.

[20] M. Walter, D. Shutin, and U.-C. Fiebig, "Delay-dependent Doppler probability density functions for vehicle-to-vehicle scatter channels," IEEE Trans. Antennas Propag., vol. 62, no. 4, pp. 2238-2249, Apr. 2014.

[21] M. Walter, D. Shutin, and A. Dammann, "Time-variant Doppler PDFs and characteristic functions for the vehicle-to-vehicle channel," IEEE Trans. Veh. Technol., vol. 66, no. 12, pp. 10 748-10763, Dec. 2017.

[22] C. A. Gutiérrez, M. Pätzold, N. M. Ortega, C. Azurdia-Meza, and F. M. Maciel-Barboza, "Doppler shift characterization of wideband mobile radio channels," IEEE Trans. Veh. Technol., pp. 1-1, 2019.

[23] M. Walter, D. Shutin, and U.-C. Fiebig, "Joint delay Doppler probability density functions for air-to-air channels," Int. J. Antennas Propagat., vol. 2014, pp. 1-11, 2014.
[24] M. Walter, U.-C. Fiebig, and A. Zajić, "Experimental verification of the non-stationary statistical model for V2V scatter channels," in Proc. IEEE 80th Veh. Technol. Conf., Vancouver, BC, Canada, Sep. 2014, pp. $1-5$.

[25] M. Walter, T. Zemen, and D. Shutin, "Empirical relationship between local scattering function and joint probability density function," in Proc. IEEE 26th Int. Symp. Pers., Indoor and Mobile Radio Commun., Hong Kong, China, Aug. 2015.

[26] M. Walter, "Scattering in non-stationary mobile-to-mobile communications channels," Ph.D. dissertation, Universität Ulm, 2016.

[27] M. Walter and M. Schnell, "The Doppler-delay characteristic of the aeronautical scatter channel," in Proc. IEEE 74th Veh. Technol. Conf., San Francisco, CA, USA, Sep. 2011, pp. 1-5.

[28] G. Sommerkorn, M. Käske, D. Czaniera, C. Schneider, G. Del Galdo, R. S. Thomä, and M. Walter, "Experimental and analytical characterization of time-variant $\mathrm{V} 2 \mathrm{~V}$ channels in a highway scenario," in Proc. 13th Eur. Conf. on Antennas and Propag. (EuCAP), 2019, pp. 1-5.

[29] M. Walter, D. Shutin, and U.-C. Fiebig, "Prolate spheroidal coordinates for modeling mobile-to-mobile channels," IEEE Antennas Wireless Propag. Lett., vol. 14, pp. 155-158, 2015.

[30] M. Walter, D. Shutin, and A. Dammann, "Algebraic analysis of the poles in the Doppler spectrum for vehicle-to-vehicle channels," IEEE Wireless Commun. Lett., vol. 7, no. 4, pp. 662-665, Aug. 2018.

[31] G. A. Deschamps, "Electromagnetics and differential forms," Proceedings of the IEEE, vol. 69, no. 6, pp. 676-696, jun 1981.

[32] K. F. Warnick and P. H. Russer, "Differential forms and electromagnetic field theory," Progress In Electromagnetics Research, vol. 148, pp. 83112, 2014.

[33] D. Bachman, A Geometric Approach to Differential Forms. Birkhäuser Boston, 2012

[34] C. Flammer, Spheroidal Wave Functions. Stanford, CA: Stanford University Press, 1957.

[35] A. Papoulis and S. U. Pillai, Probability, Random Variables, and Stochastic Processes, ser. McGraw-Hill electrical and electronic engineering series. McGraw-Hill, 2002.

[36] H. Hilton, Plane algebraic curves. The Clarendon press, 1920.

[37] E. Kunz and R. Belshoff, Introduction to Plane Algebraic Curves. Birkhäuser Boston, 2005.

[38] S. S. Abhyankar, Algebraic Geometry for Scientists and Engineers, ser. Mathematical Surveys and Monographs. American Mathematical Society, 1990

[39] E. Cartan, "Sur certaines expressions différentielles et le problème de Pfaff," Annales scientifiques de l'École Normale Supérieure, vol. 3e série, 16, pp. 239-332, 1899.

[40] M. Abramowitz and I. A. Stegun, Handbook of Mathematical Functions: With Formulas, Graphs, and Mathematical Tables, ser. Applied mathematics series. Dover Publications, 1965.

[41] C. Misner, K. Thorne, J. Wheeler, and D. Kaiser, Gravitation. Princeton University Press, 2017.

[42] M. Pätzold, Mobile Fading Channels. Chichester, UK: Wiley, 2002.

[43] T. R. Hagedorn, "General formulas for solving solvable sextic equations," Journal of Algebra, vol. 233, no. 2, pp. 704 - 757, 2000.

[44] M. I. Skolnik, "An analysis of bistatic radar," IRE Trans. Aerosp. and Navigational Electron., vol. ANE-8, no. 1, pp. 19-27, Mar. 1961

[45] J. van Rees, "Measurements of the wide-band radio channel characteristics for rural, residential, and suburban areas," IEEE Trans. Veh. Technol., vol. 36, no. 1, pp. 2-6, Feb. 1987.

[46] S. Y. Seidel, T. S. Rappaport, S. Jain, M. L. Lord, and R. Singh, "Path loss, scattering and multipath delay statistics in four European cities for digital cellular and microcellular radiotelephone," IEEE Trans. Veh. Technol., vol. 40, no. 4, pp. 721-730, Nov. 1991.

[47] M. Skolnik, Radar Handbook, Third Edition, ser. Electronics electrical engineering. McGraw-Hill Education, 2008.

[48] P. A. Bello, "Aeronautical channel characterization," IEEE Trans. Commun., vol. 21, no. 5, pp. 548-563, May 1973.

[49] M. Walter, D. Shutin, A. Dammann, and D. W. Matolak, "Modeling of highly non-stationary low altitude aircraft-to-aircraft channels," in Proc. IEEE Mil. Commun. Conf. (MILCOM), Los Angeles, CA, USA, Oct. 2018.

[50] D. W. Matolak and U.-C. Fiebig, "UAV channel models: Review and future research," in Proc. 13th Eur. Conf. on Antennas and Propag. (EuCAP), Mar. 2019, pp. 1-5. 


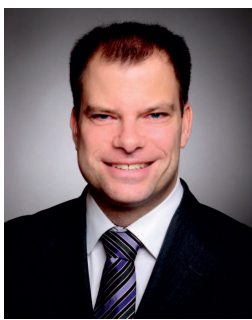

Michael Walter (M'10-SM'17) received his Dipl.Ing. and Dr.-Ing. degree in electrical engineering from Ulm University, Germany, in 2008 and 2015.

Since 2009 he has been a member of the scientific staff at the Institute of Communications and Navigation at the German Aerospace Center (DLR) in Oberpfaffenhofen, Germany, where he gained extensive experience in organizing and conducting numerous measurement campaigns for vehicular and aeronautical research. He was a visiting scholar at the School of Electrical and Computer Engineering at the Georgia Institute of Technology in 2014. His research interests include wireless communications, modeling of mobile-to-mobile channels, and channel coding.

Dr. Walter was the recipient of the IEEE/AIAA David Lubkowski Memorial for Advancement in Digital Avionics Best Paper Award of the 29th DASC in 2011.

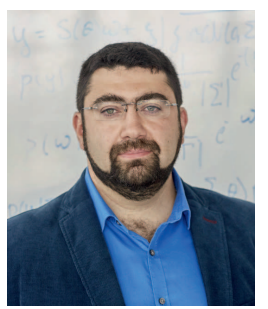

Dmitriy Shutin (M'06-SM'12) received his Master degree in Computer Science in 2000 from Dniepropetrovsk State University, Ukraine, and the Ph.D. degree in electrical engineering from Graz University of Technology, Graz, Austria, in 2006.

During 2001-2006 and 2006-2009, he was a Teaching Assistant and an Assistant Professor, respectively, with the Signal Processing and Speech Communication Laboratory, Graz University of Technology. From 2009 to 2011 he was a Research Associate with the Department of Electrical Engineering at Princeton University, Princeton, NJ, USA. Since 2011 he is with the Institute of Communications and Navigation, German Aerospace Center His current research interests include modeling and estimation of the radio propagation channels, statistical signal processing, radar signal processing, and machine learning for signal processing.

Dr. Shutin was a recipient of the Best Student Paper Award at the 2005 IEEE International Conference on Information, Communications and Signal Processing (ICICS). In 2009 he was awarded the Erwin Schroedinger Research Fellowship.

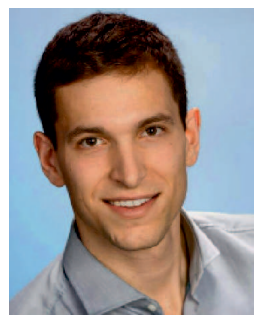

Martin Schmidhammer (M'19) studied electrical engineering at the Technical University of Munich (TUM) and completed his master's degree with high distinction in 2016. He wrote his master's thesis "Deep learning for virtual view generation" at Navvis $\mathrm{GmbH}$ and the TUM Chair of Media Technology. Thereafter he joined the Vehicular Applications Group at DLR's Institute of Communications and Navigation, where he has been researching on V2X communications and localization with V2X signals. Currently he is working towards his Ph.D. in the field of RF based detection and localization of non-cooperative users.

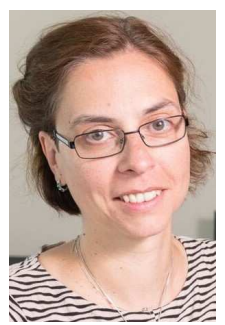

Alenka Zajic (S'99-M'09-SM'13) received the B.Sc. and M.Sc. degrees form the School of Electrical Engineering, University of Belgrade, in 2001 and 2003, respectively. She received her Ph.D. degree in Electrical and Computer Engineering from the Georgia Institute of Technology in 2008. Currently, she is an Associate Professor in the School of Electrical and Computer Engineering at Georgia Institute of Technology. Prior to that, she was a visiting faculty member in the School of Computer Science at Georgia Institute of Technology, a post-doctoral fellow in the Naval Research Laboratory, and a design engineer at Skyworks Solutions Inc. Her research interests span areas of electromagnetic, wireless communications, signal processing, and computer engineering.

Dr. Zajic was the recipient of the 2017 NSF CAREER award, the Best Paper Award at MICRO 2016, 2012 Neal Shepherd Memorial Best Propagation Paper Award, the Best Student Paper Award at the IEEE International Conference on Communications and Electronics 2014, the Best Paper Award at the International Conference on Telecommunications 2008, the Best Student Paper Award at the 2007 Wireless Communications and Networking Conference,and the Dan Noble Fellowship in 2004, which was awarded by Motorola Inc.and the IEEE Vehicular Technology Society for quality impact in the area of vehicular technology.
David W. Matolak (S'82-M'83-SM'00) received the B.S. degree from The Pennsylvania State University, M.S. degree from The University of Massachusetts, and Ph.D. degree from The University of Virginia, all in electrical engineering. He has over 25 years' experience in communication system research, development, and deployment, with industry, government institutions, and academia, including AT\&T Bell Labs, L3 Communication Systems, MITRE, and Lockheed Martin. He has over 250 publications and multiple patents. He was a professor at Ohio University (1999-2012), and since 2012 has been a professor at the University of South Carolina. His research interests are radio channel modeling and PHY/MAC communication techniques for statistically non-stationary fading channels. Prof. Matolak is also a member of Eta Kappa Nu, Sigma Xi, Tau Beta Pi, URSI, ASEE, and AIAA. 


\section{General PSCS Polynomial in (31)}

$g(\eta)=$

$\left(B^{4} v_{\mathrm{r} x}^{2} \xi^{6}+C^{4} v_{\mathrm{r} x}^{2} \xi^{6}+A^{2} B^{2} v_{\mathrm{r} x}^{2} \xi^{6}+A^{2} C^{2} v_{\mathrm{r} x}^{2} \xi^{6}+2 B^{2} C^{2} v_{\mathrm{r} x}^{2} \xi^{6}+A^{4} v_{\mathrm{r} y}^{2} \xi^{6}+C^{4} v_{\mathrm{r} y}^{2} \xi^{6}+A^{2} B^{2} v_{\mathrm{r} y}^{2} \xi^{6}+2 A^{2} C^{2} v_{\mathrm{r} y}^{2} \xi^{6}\right.$ $+B^{2} C^{2} v_{\mathrm{r} y}^{2} \xi^{6}+A^{4} v_{\mathrm{r} z}^{2} \xi^{6}+B^{4} v_{\mathrm{r} z}^{2} \xi^{6}+2 A^{2} B^{2} v_{\mathrm{r} z}^{2} \xi^{6}+A^{2} C^{2} v_{\mathrm{r} z}^{2} \xi^{6}+B^{2} C^{2} v_{\mathrm{r} z}^{2} \xi^{6}+B^{4} v_{\mathrm{t} x}^{2} \xi^{6}+C^{4} v_{\mathrm{t} x}^{2} \xi^{6}+A^{2} B^{2} v_{\mathrm{t} x}^{2} \xi^{6}$ $+A^{2} C^{2} v_{\mathrm{t} x}^{2} \xi^{6}+2 B^{2} C^{2} v_{\mathrm{t} x}^{2} \xi^{6}+A^{4} v_{\mathrm{t} y}^{2} \xi^{6}+C^{4} v_{\mathrm{t} y}^{2} \xi^{6}+A^{2} B^{2} v_{\mathrm{t} y}^{2} \xi^{6}+2 A^{2} C^{2} v_{\mathrm{t} y}^{2} \xi^{6}+B^{2} C^{2} v_{\mathrm{t} y}^{2} \xi^{6}+A^{4} v_{\mathrm{t} z}^{2} \xi^{6}+B^{4} v_{\mathrm{t} z}^{2} \xi^{6}$ $+2 A^{2} B^{2} v_{\mathrm{t} z}^{2} \xi^{6}+A^{2} C^{2} v_{\mathrm{t} z}^{2} \xi^{6}+B^{2} C^{2} v_{\mathrm{t} z}^{2} \xi^{6}-2 A B^{3} v_{\mathrm{r} x} v_{\mathrm{r} y} \xi^{6}-2 A B C^{2} v_{\mathrm{r} x} v_{\mathrm{r} y} \xi^{6}-2 A^{3} B v_{\mathrm{r} x} v_{\mathrm{r} y} \xi^{6}-2 A C^{3} v_{\mathrm{r} x} v_{\mathrm{r} z} \xi^{6}$ $-2 A^{3} C v_{\mathrm{r} x} v_{\mathrm{r} z} \xi^{6}-2 A B^{2} C v_{\mathrm{r} x} v_{\mathrm{r} z} \xi^{6}-2 B C^{3} v_{\mathrm{r} y} v_{\mathrm{r} z} \xi^{6}-2 B^{3} C v_{\mathrm{r} y} v_{\mathrm{r} z} \xi^{6}-2 A^{2} B C v_{\mathrm{r} y} v_{\mathrm{r} z} \xi^{6}+2 B^{4} v_{\mathrm{r} x} v_{\mathrm{t} x} \xi^{6}+2 C^{4} v_{\mathrm{r} x} v_{\mathrm{t} x} \xi^{6}$ $+2 A^{2} B^{2} v_{\mathrm{r} x} v_{\mathrm{t} x} \xi^{6}+2 A^{2} C^{2} v_{\mathrm{r} x} v_{\mathrm{t} x} \xi^{6}+4 B^{2} C^{2} v_{\mathrm{r} x} v_{\mathrm{t} x} \xi^{6}-2 A B^{3} v_{\mathrm{r} y} v_{\mathrm{t} x} \xi^{6}-2 A B C^{2} v_{\mathrm{r} y} v_{\mathrm{t} x} \xi^{6}-2 A^{3} B v_{\mathrm{r} y} v_{\mathrm{t} x} \xi^{6}$ $-2 A C^{3} v_{\mathrm{r} z} v_{\mathrm{t} x} \xi^{6}-2 A^{3} C v_{\mathrm{r} z} v_{\mathrm{t} x} \xi^{6}-2 A B^{2} C v_{\mathrm{r} z} v_{\mathrm{t} x} \xi^{6}-2 A B^{3} v_{\mathrm{r} x} v_{\mathrm{t} y} \xi^{6}-2 A B C^{2} v_{\mathrm{r} x} v_{\mathrm{t} y} \xi^{6}-2 A^{3} B v_{\mathrm{r} x} v_{\mathrm{t} y} \xi^{6}+2 A^{4} v_{\mathrm{r} y} v_{\mathrm{t} y} \xi^{6}$ $+2 C^{4} v_{\mathrm{r} y} v_{\mathrm{t} y} \xi^{6}+2 A^{2} B^{2} v_{\mathrm{r} y} v_{\mathrm{t} y} \xi^{6}+4 A^{2} C^{2} v_{\mathrm{r} y} v_{\mathrm{t} y} \xi^{6}+2 B^{2} C^{2} v_{\mathrm{r} y} v_{\mathrm{t} y} \xi^{6}-2 B C^{3} v_{\mathrm{r} z} v_{\mathrm{t} y} \xi^{6}-2 B^{3} C v_{\mathrm{r} z} v_{\mathrm{t} y} \xi^{6}$ $-2 A^{2} B C v_{\mathrm{r} z} v_{\mathrm{t} y} \xi^{6}-2 A B^{3} v_{\mathrm{t} x} v_{\mathrm{t} y} \xi^{6}-2 A B C^{2} v_{\mathrm{t} x} v_{\mathrm{t} y} \xi^{6}-2 A^{3} B v_{\mathrm{t} x} v_{\mathrm{t} y} \xi^{6}-2 A C^{3} v_{\mathrm{r} x} v_{\mathrm{t} z} \xi^{6}-2 A^{3} C v_{\mathrm{r} x} v_{\mathrm{t} z} \xi^{6}$ $-2 A B^{2} C v_{\mathrm{r} x} v_{\mathrm{t} z} \xi^{6}-2 B C^{3} v_{\mathrm{r} y} v_{\mathrm{t} z} \xi^{6}-2 B^{3} C v_{\mathrm{r} y} v_{\mathrm{t} z} \xi^{6}-2 A^{2} B C v_{\mathrm{r} y} v_{\mathrm{t} z} \xi^{6}+2 A^{4} v_{\mathrm{r} z} v_{\mathrm{t} z} \xi^{6}+2 B^{4} v_{\mathrm{r} z} v_{\mathrm{t} z} \xi^{6}+4 A^{2} B^{2} v_{\mathrm{r} z} v_{\mathrm{t} z} \xi^{6}$ $+2 A^{2} C^{2} v_{\mathrm{r} z} v_{\mathrm{t} z} \xi^{6}+2 B^{2} C^{2} v_{\mathrm{r} z} v_{\mathrm{t} z} \xi^{6}-2 A C^{3} v_{\mathrm{t} x} v_{\mathrm{t} z} \xi^{6}-2 A^{3} C v_{\mathrm{t} x} v_{\mathrm{t} z} \xi^{6}-2 A B^{2} C v_{\mathrm{t} x} v_{\mathrm{t} z} \xi^{6}-2 B C^{3} v_{\mathrm{t} y} v_{\mathrm{t} z} \xi^{6}$ $-2 B^{3} C v_{\mathrm{t} y} v_{\mathrm{t} z} \xi^{6}-2 A^{2} B C v_{\mathrm{t} y} v_{\mathrm{t} z} \xi^{6}-2 B^{4} v_{\mathrm{r} x}^{2} \xi^{4}-2 D C^{3} v_{\mathrm{r} x}^{2} \xi^{4}-2 A^{2} B^{2} v_{\mathrm{r} x}^{2} \xi^{4}-A^{2} C^{2} v_{\mathrm{r} x}^{2} \xi^{4}-2 B^{2} C^{2} v_{\mathrm{r} x}^{2} \xi^{4}-2 A^{2} D C v_{\mathrm{r} x}^{2} \xi^{4}$ $-2 B^{2} D C v_{\mathrm{r} x}^{2} \xi^{4}-2 A^{4} v_{\mathrm{r} y}^{2} \xi^{4}-2 D C^{3} v_{\mathrm{r} y}^{2} \xi^{4}-2 A^{2} B^{2} v_{\mathrm{r} y}^{2} \xi^{4}-2 A^{2} C^{2} v_{\mathrm{r} y}^{2} \xi^{4}-B^{2} C^{2} v_{\mathrm{r} y}^{2} \xi^{4}-2 A^{2} D C v_{\mathrm{r} y}^{2} \xi^{4}-2 B^{2} D C v_{\mathrm{r} y}^{2} \xi^{4}$ $-3 A^{4} v_{\mathrm{r} z}^{2} \xi^{4}-3 B^{4} v_{\mathrm{r} z}^{2} \xi^{4}-6 A^{2} B^{2} v_{\mathrm{r} z}^{2} \xi^{4}-2 A^{2} C^{2} v_{\mathrm{r} z}^{2} \xi^{4}-2 B^{2} C^{2} v_{\mathrm{r} z}^{2} \xi^{4}-2 B^{4} v_{\mathrm{t} x}^{2} \xi^{4}+2 D C^{3} v_{\mathrm{t} x}^{2} \xi^{4}-2 A^{2} B^{2} v_{\mathrm{t} x}^{2} \xi^{4}$ $-A^{2} C^{2} v_{\mathrm{t} x}^{2} \xi^{4}-2 B^{2} C^{2} v_{\mathrm{t} x}^{2} \xi^{4}+2 A^{2} D C v_{\mathrm{t} x}^{2} \xi^{4}+2 B^{2} D C v_{\mathrm{t} x}^{2} \xi^{4}-2 A^{4} v_{\mathrm{t} y}^{2} \xi^{4}+2 D C^{3} v_{\mathrm{t} y}^{2} \xi^{4}-2 A^{2} B^{2} v_{\mathrm{t} y}^{2} \xi^{4}-2 A^{2} C^{2} v_{\mathrm{t} y}^{2} \xi^{4}$ $-B^{2} C^{2} v_{\mathrm{t} y}^{2} \xi^{4}+2 A^{2} D C v_{\mathrm{t} y}^{2} \xi^{4}+2 B^{2} D C v_{\mathrm{t} y}^{2} \xi^{4}-3 A^{4} v_{\mathrm{t} z}^{2} \xi^{4}-3 B^{4} v_{\mathrm{t} z}^{2} \xi^{4}-6 A^{2} B^{2} v_{\mathrm{t} z}^{2} \xi^{4}-2 A^{2} C^{2} v_{\mathrm{t} z}^{2} \xi^{4}-2 B^{2} C^{2} v_{\mathrm{t} z}^{2} \xi^{4}$ $+4 A B^{3} v_{\mathrm{r} x} v_{\mathrm{r} y} \xi^{4}+2 A B C^{2} v_{\mathrm{r} x} v_{\mathrm{r} y} \xi^{4}+4 A^{3} B v_{\mathrm{r} x} v_{\mathrm{r} y} \xi^{4}+2 A C^{3} v_{\mathrm{r} x} v_{\mathrm{r} z} \xi^{4}+2 A D C^{2} v_{\mathrm{r} x} v_{\mathrm{r} z} \xi^{4}+2 A^{3} D v_{\mathrm{r} x} v_{\mathrm{r} z} \xi^{4}$ $+2 A B^{2} D v_{\mathrm{r} x} v_{\mathrm{r} z} \xi^{4}+4 A^{3} C v_{\mathrm{r} x} v_{\mathrm{r} z} \xi^{4}+4 A B^{2} C v_{\mathrm{r} x} v_{\mathrm{r} z} \xi^{4}+2 B C^{3} v_{\mathrm{r} y} v_{\mathrm{r} z} \xi^{4}+2 B D C^{2} v_{\mathrm{r} y} v_{\mathrm{r} z} \xi^{4}+2 B^{3} D v_{\mathrm{r} y} v_{\mathrm{r} z} \xi^{4}$ $+2 A^{2} B D v_{\mathrm{r} y} v_{\mathrm{r} z} \xi^{4}+4 B^{3} C v_{\mathrm{r} y} v_{\mathrm{r} z} \xi^{4}+4 A^{2} B C v_{\mathrm{r} y} v_{\mathrm{r} z} \xi^{4}-4 B^{4} v_{\mathrm{r} x} v_{\mathrm{t} x} \xi^{4}-4 A^{2} B^{2} v_{\mathrm{r} x} v_{\mathrm{t} x} \xi^{4}-2 A^{2} C^{2} v_{\mathrm{r} x} v_{\mathrm{t} x} \xi^{4}$ $-4 B^{2} C^{2} v_{\mathrm{r} x} v_{\mathrm{t} x} \xi^{4}+4 A B^{3} v_{\mathrm{r} y} v_{\mathrm{t} x} \xi^{4}+2 A B C^{2} v_{\mathrm{r} y} v_{\mathrm{t} x} \xi^{4}+4 A^{3} B v_{\mathrm{r} y} v_{\mathrm{t} x} \xi^{4}+2 A C^{3} v_{\mathrm{r} z} v_{\mathrm{t} x} \xi^{4}-2 A D C^{2} v_{\mathrm{r} z} v_{\mathrm{t} x} \xi^{4}$ $-2 A^{3} D v_{\mathrm{r} z} v_{\mathrm{t} x} \xi^{4}-2 A B^{2} D v_{\mathrm{r} z} v_{\mathrm{t} x} \xi^{4}+4 A^{3} C v_{\mathrm{r} z} v_{\mathrm{t} x} \xi^{4}+4 A B^{2} C v_{\mathrm{r} z} v_{\mathrm{t} x} \xi^{4}+4 A B^{3} v_{\mathrm{r} x} v_{\mathrm{t} y} \xi^{4}+2 A B C^{2} v_{\mathrm{r} x} v_{\mathrm{t} y} \xi^{4}$ $+4 A^{3} B v_{\mathrm{r} x} v_{\mathrm{t} y} \xi^{4}-4 A^{4} v_{\mathrm{r} y} v_{\mathrm{t} y} \xi^{4}-4 A^{2} B^{2} v_{\mathrm{r} y} v_{\mathrm{t} y} \xi^{4}-4 A^{2} C^{2} v_{\mathrm{r} y} v_{\mathrm{t} y} \xi^{4}-2 B^{2} C^{2} v_{\mathrm{r} y} v_{\mathrm{t} y} \xi^{4}+2 B C^{3} v_{\mathrm{r} z} v_{\mathrm{t} y} \xi^{4}$ $-2 B D C^{2} v_{\mathrm{r} z} v_{\mathrm{t} y} \xi^{4}-2 B^{3} D v_{\mathrm{r} z} v_{\mathrm{t} y} \xi^{4}-2 A^{2} B D v_{\mathrm{r} z} v_{\mathrm{t} y} \xi^{4}+4 B^{3} C v_{\mathrm{r} z} v_{\mathrm{t} y} \xi^{4}+4 A^{2} B C v_{\mathrm{r} z} v_{\mathrm{t} y} \xi^{4}+4 A B^{3} v_{\mathrm{t} x} v_{\mathrm{t} y} \xi^{4}$ $+2 A B C^{2} v_{\mathrm{t} x} v_{\mathrm{t} y} \xi^{4}+4 A^{3} B v_{\mathrm{t} x} v_{\mathrm{t} y} \xi^{4}+2 A C^{3} v_{\mathrm{r} x} v_{\mathrm{t} z} \xi^{4}+2 A D C^{2} v_{\mathrm{r} x} v_{\mathrm{t} z} \xi^{4}+2 A^{3} D v_{\mathrm{r} x} v_{\mathrm{t} z} \xi^{4}+2 A B^{2} D v_{\mathrm{r} x} v_{\mathrm{t} z} \xi^{4}$ $+4 A^{3} C v_{\mathrm{r} x} v_{\mathrm{t} z} \xi^{4}+4 A B^{2} C v_{\mathrm{r} x} v_{\mathrm{t} z} \xi^{4}+2 B C^{3} v_{\mathrm{r} y} v_{\mathrm{t} z} \xi^{4}+2 B D C^{2} v_{\mathrm{r} y} v_{\mathrm{t} z} \xi^{4}+2 B^{3} D v_{\mathrm{r} y} v_{\mathrm{t} z} \xi^{4}+2 A^{2} B D v_{\mathrm{r} y} v_{\mathrm{t} z} \xi^{4}$ $+4 B^{3} C v_{\mathrm{r} y} v_{\mathrm{t} z} \xi^{4}+4 A^{2} B C v_{\mathrm{r} y} v_{\mathrm{t} z} \xi^{4}-6 A^{4} v_{\mathrm{r} z} v_{\mathrm{t} z} \xi^{4}-6 B^{4} v_{\mathrm{r} z} v_{\mathrm{t} z} \xi^{4}-12 A^{2} B^{2} v_{\mathrm{r} z} v_{\mathrm{t} z} \xi^{4}-4 A^{2} C^{2} v_{\mathrm{r} z} v_{\mathrm{t} z} \xi^{4}$ $-4 B^{2} C^{2} v_{\mathrm{r} z} v_{\mathrm{t} z} \xi^{4}+2 A C^{3} v_{\mathrm{t} x} v_{\mathrm{t} z} \xi^{4}-2 A D C^{2} v_{\mathrm{t} x} v_{\mathrm{t} z} \xi^{4}-2 A^{3} D v_{\mathrm{t} x} v_{\mathrm{t} z} \xi^{4}-2 A B^{2} D v_{\mathrm{t} x} v_{\mathrm{t} z} \xi^{4}+4 A^{3} C v_{\mathrm{t} x} v_{\mathrm{t} z} \xi^{4}$ $+4 A B^{2} C v_{\mathrm{t} x} v_{\mathrm{t} z} \xi^{4}+2 B C^{3} v_{\mathrm{t} y} v_{\mathrm{t} z} \xi^{4}-2 B D C^{2} v_{\mathrm{t} y} v_{\mathrm{t} z} \xi^{4}-2 B^{3} D v_{\mathrm{t} y} v_{\mathrm{t} z} \xi^{4}-2 A^{2} B D v_{\mathrm{t} y} v_{\mathrm{t} z} \xi^{4}+4 B^{3} C v_{\mathrm{t} y} v_{\mathrm{t} z} \xi^{4}$ $+4 A^{2} B C v_{\mathrm{t} y} v_{\mathrm{t} z} \xi^{4}+B^{4} v_{\mathrm{r} x}^{2} \xi^{2}+A^{2} B^{2} v_{\mathrm{r} x}^{2} \xi^{2}+A^{2} D^{2} v_{\mathrm{r} x}^{2} \xi^{2}+D^{2} C^{2} v_{\mathrm{r} x}^{2} \xi^{2}+2 A^{2} D C v_{\mathrm{r} x}^{2} \xi^{2}+2 B^{2} D C v_{\mathrm{r} x}^{2} \xi^{2}+A^{4} v_{\mathrm{r} y}^{2} \xi^{2}$ $+A^{2} B^{2} v_{\mathrm{r} y}^{2} \xi^{2}+B^{2} D^{2} v_{\mathrm{r} y}^{2} \xi^{2}+D^{2} C^{2} v_{\mathrm{r} y}^{2} \xi^{2}+2 A^{2} D C v_{\mathrm{r} y}^{2} \xi^{2}+2 B^{2} D C v_{\mathrm{r} y}^{2} \xi^{2}+3 A^{4} v_{\mathrm{r} z}^{2} \xi^{2}+3 B^{4} v_{\mathrm{r} z}^{2} \xi^{2}+6 A^{2} B^{2} v_{\mathrm{r} z}^{2} \xi^{2}$ $+A^{2} C^{2} v_{\mathrm{r} z}^{2} \xi^{2}+B^{2} C^{2} v_{\mathrm{r} z}^{2} \xi^{2}+B^{4} v_{\mathrm{t} x}^{2} \xi^{2}+A^{2} B^{2} v_{\mathrm{tx}}^{2} \xi^{2}+A^{2} D^{2} v_{\mathrm{t} x}^{2} \xi^{2}+D^{2} C^{2} v_{\mathrm{t} x}^{2} \xi^{2}-2 A^{2} D C v_{\mathrm{t} x}^{2} \xi^{2}-2 B^{2} D C v_{\mathrm{t} x}^{2} \xi^{2}$ $+A^{4} v_{\mathrm{t} y}^{2} \xi^{2}+A^{2} B^{2} v_{\mathrm{t} y}^{2} \xi^{2}+B^{2} D^{2} v_{\mathrm{t} y}^{2} \xi^{2}+D^{2} C^{2} v_{\mathrm{t} y}^{2} \xi^{2}-2 A^{2} D C v_{\mathrm{t} y}^{2} \xi^{2}-2 B^{2} D C v_{\mathrm{t} y}^{2} \xi^{2}+3 A^{4} v_{\mathrm{t} z}^{2} \xi^{2}+3 B^{4} v_{\mathrm{t} z}^{2} \xi^{2}$ $+6 A^{2} B^{2} v_{\mathrm{t} z}^{2} \xi^{2}+A^{2} C^{2} v_{\mathrm{t} z}^{2} \xi^{2}+B^{2} C^{2} v_{\mathrm{t} z}^{2} \xi^{2}-2 A B^{3} v_{\mathrm{r} x} v_{\mathrm{r} y} \xi^{2}+2 A B D^{2} v_{\mathrm{r} x} v_{\mathrm{r} y} \xi^{2}-2 A^{3} B v_{\mathrm{r} x} v_{\mathrm{r} y} \xi^{2}-2 A D C^{2} v_{\mathrm{r} x} v_{\mathrm{r} z} \xi^{2}$ $-4 A^{3} D v_{\mathrm{r} x} v_{\mathrm{r} z} \xi^{2}-4 A B^{2} D v_{\mathrm{r} x} v_{\mathrm{r} z} \xi^{2}-2 A^{3} C v_{\mathrm{r} x} v_{\mathrm{r} z} \xi^{2}-2 A B^{2} C v_{\mathrm{r} x} v_{\mathrm{r} z} \xi^{2}-2 B D C^{2} v_{\mathrm{r} y} v_{\mathrm{r} z} \xi^{2}-4 B^{3} D v_{\mathrm{r} y} v_{\mathrm{r} z} \xi^{2}$ $-4 A^{2} B D v_{\mathrm{r} y} v_{\mathrm{r} z} \xi^{2}-2 B^{3} C v_{\mathrm{r} y} v_{\mathrm{r} z} \xi^{2}-2 A^{2} B C v_{\mathrm{r} y} v_{\mathrm{r} z} \xi^{2}+2 B^{4} v_{\mathrm{r} x} v_{\mathrm{t} x} \xi^{2}+2 A^{2} B^{2} v_{\mathrm{r} x} v_{\mathrm{t} x} \xi^{2}-2 A^{2} D^{2} v_{\mathrm{r} x} v_{\mathrm{t} x} \xi^{2}$ $-2 D^{2} C^{2} v_{\mathrm{r} x} v_{\mathrm{t} x} \xi^{2}-2 A B^{3} v_{\mathrm{r} y} v_{\mathrm{t} x} \xi^{2}-2 A B D^{2} v_{\mathrm{r} y} v_{\mathrm{t} x} \xi^{2}-2 A^{3} B v_{\mathrm{r} y} v_{\mathrm{t} x} \xi^{2}+2 A D C^{2} v_{\mathrm{r} z} v_{\mathrm{t} x} \xi^{2}+4 A^{3} D v_{\mathrm{r} z} v_{\mathrm{t} x} \xi^{2}$ $+4 A B^{2} D v_{\mathrm{r} z} v_{\mathrm{t} x} \xi^{2}-2 A^{3} C v_{\mathrm{r} z} v_{\mathrm{t} x} \xi^{2}-2 A B^{2} C v_{\mathrm{r} z} v_{\mathrm{t} x} \xi^{2}-2 A B^{3} v_{\mathrm{r} x} v_{\mathrm{t} y} \xi^{2}-2 A B D^{2} v_{\mathrm{r} x} v_{\mathrm{t} y} \xi^{2}-2 A^{3} B v_{\mathrm{r} x} v_{\mathrm{t} y} \xi^{2}$ $+2 A^{4} v_{\mathrm{r} y} v_{\mathrm{t} y} \xi^{2}+2 A^{2} B^{2} v_{\mathrm{r} y} v_{\mathrm{t} y} \xi^{2}-2 B^{2} D^{2} v_{\mathrm{r} y} v_{\mathrm{t} y} \xi^{2}-2 D^{2} C^{2} v_{\mathrm{r} y} v_{\mathrm{t} y} \xi^{2}+2 B D C^{2} v_{\mathrm{r} z} v_{\mathrm{t} y} \xi^{2}+4 B^{3} D v_{\mathrm{r} z} v_{\mathrm{t} y} \xi^{2}$ $+4 A^{2} B D v_{\mathrm{r} z} v_{\mathrm{t} y} \xi^{2}-2 B^{3} C v_{\mathrm{r} z} v_{\mathrm{t} y} \xi^{2}-2 A^{2} B C v_{\mathrm{r} z} v_{\mathrm{t} y} \xi^{2}-2 A B^{3} v_{\mathrm{t} x} v_{\mathrm{t} y} \xi^{2}+2 A B D^{2} v_{\mathrm{t} x} v_{\mathrm{t} y} \xi^{2}-2 A^{3} B v_{\mathrm{t} x} v_{\mathrm{t} y} \xi^{2}$ $-2 A D C^{2} v_{\mathrm{r} x} v_{\mathrm{t} z} \xi^{2}-4 A^{3} D v_{\mathrm{r} x} v_{\mathrm{t} z} \xi^{2}-4 A B^{2} D v_{\mathrm{r} x} v_{\mathrm{t} z} \xi^{2}-2 A^{3} C v_{\mathrm{r} x} v_{\mathrm{t} z} \xi^{2}-2 A B^{2} C v_{\mathrm{r} x} v_{\mathrm{t} z} \xi^{2}-2 B D C^{2} v_{\mathrm{r} y} v_{\mathrm{t} z} \xi^{2}$ 
$-4 B^{3} D v_{\mathrm{r} y} v_{\mathrm{t} z} \xi^{2}-4 A^{2} B D v_{\mathrm{r} y} v_{\mathrm{t} z} \xi^{2}-2 B^{3} C v_{\mathrm{r} y} v_{\mathrm{t} z} \xi^{2}-2 A^{2} B C v_{\mathrm{r} y} v_{\mathrm{t} z} \xi^{2}+6 A^{4} v_{\mathrm{r} z} v_{\mathrm{t} z} \xi^{2}+6 B^{4} v_{\mathrm{r} z} v_{\mathrm{t} z} \xi^{2}+12 A^{2} B^{2} v_{\mathrm{r} z} v_{\mathrm{t} z} \xi^{2}$ $+2 A^{2} C^{2} v_{\mathrm{r} z} v_{\mathrm{t} z} \xi^{2}+2 B^{2} C^{2} v_{\mathrm{r} z} v_{\mathrm{t} z} \xi^{2}+2 A D C^{2} v_{\mathrm{t} x} v_{\mathrm{t} z} \xi^{2}+4 A^{3} D v_{\mathrm{t} x} v_{\mathrm{t} z} \xi^{2}+4 A B^{2} D v_{\mathrm{t} x} v_{\mathrm{t} z} \xi^{2}-2 A^{3} C v_{\mathrm{t} x} v_{\mathrm{t} z} \xi^{2}$

$-2 A B^{2} C v_{\mathrm{t} x} v_{\mathrm{t} z} \xi^{2}+2 B D C^{2} v_{\mathrm{t} y} v_{\mathrm{t} z} \xi^{2}+4 B^{3} D v_{\mathrm{t} y} v_{\mathrm{t} z} \xi^{2}+4 A^{2} B D v_{\mathrm{t} y} v_{\mathrm{t} z} \xi^{2}-2 B^{3} C v_{\mathrm{t} y} v_{\mathrm{t} z} \xi^{2}-2 A^{2} B C v_{\mathrm{t} y} v_{\mathrm{t} z} \xi^{2}-A^{2} D^{2} v_{\mathrm{r} x}^{2}$ $-B^{2} D^{2} v_{\mathrm{r} y}^{2}-A^{4} v_{\mathrm{r} z}^{2}-B^{4} v_{\mathrm{r} z}^{2}-2 A^{2} B^{2} v_{\mathrm{r} z}^{2}-A^{2} D^{2} v_{\mathrm{t} x}^{2}-B^{2} D^{2} v_{\mathrm{t} y}^{2}-A^{4} v_{\mathrm{t} z}^{2}-B^{4} v_{\mathrm{t} z}^{2}-2 A^{2} B^{2} v_{\mathrm{t} z}^{2}-2 A B D^{2} v_{\mathrm{r} x} v_{\mathrm{r} y}$ $+2 A^{3} D v_{\mathrm{r} x} v_{\mathrm{r} z}+2 A B^{2} D v_{\mathrm{r} x} v_{\mathrm{r} z}+2 B^{3} D v_{\mathrm{r} y} v_{\mathrm{r} z}+2 A^{2} B D v_{\mathrm{r} y} v_{\mathrm{r} z}+2 A^{2} D^{2} v_{\mathrm{r} x} v_{\mathrm{t} x}+2 A B D^{2} v_{\mathrm{r} y} v_{\mathrm{t} x}-2 A^{3} D v_{\mathrm{r} z} v_{\mathrm{t} x}$ $-2 A B^{2} D v_{\mathrm{r} z} v_{\mathrm{t} x}+2 A B D^{2} v_{\mathrm{r} x} v_{\mathrm{t} y}+2 B^{2} D^{2} v_{\mathrm{r} y} v_{\mathrm{t} y}-2 B^{3} D v_{\mathrm{r} z} v_{\mathrm{t} y}-2 A^{2} B D v_{\mathrm{r} z} v_{\mathrm{t} y}-2 A B D^{2} v_{\mathrm{t} x} v_{\mathrm{t} y}+2 A^{3} D v_{\mathrm{r} x} v_{\mathrm{t} z}$ $+2 A B^{2} D v_{\mathrm{r} x} v_{\mathrm{t} z}+2 B^{3} D v_{\mathrm{r} y} v_{\mathrm{t} z}+2 A^{2} B D v_{\mathrm{r} y} v_{\mathrm{t} z}-2 A^{4} v_{\mathrm{r} z} v_{\mathrm{t} z}-2 B^{4} v_{\mathrm{r} z} v_{\mathrm{t} z}-4 A^{2} B^{2} v_{\mathrm{r} z} v_{\mathrm{t} z}-2 A^{3} D v_{\mathrm{t} x} v_{\mathrm{t} z}-2 A B^{2} D v_{\mathrm{t} x} v_{\mathrm{t} z}$ $\left.-2 B^{3} D v_{\mathrm{t} y} v_{\mathrm{t} z}-2 A^{2} B D v_{\mathrm{t} y} v_{\mathrm{t} z}\right) \eta^{6}$ 
$+\left(4 B^{4} v_{\mathrm{r} x}^{2} \xi^{7}+4 C^{4} v_{\mathrm{r} x}^{2} \xi^{7}+4 A^{2} B^{2} v_{\mathrm{r} x}^{2} \xi^{7}+4 A^{2} C^{2} v_{\mathrm{r} x}^{2} \xi^{7}+8 B^{2} C^{2} v_{\mathrm{r} x}^{2} \xi^{7}+4 A^{4} v_{\mathrm{r} y}^{2} \xi^{7}+4 C^{4} v_{\mathrm{r} y}^{2} \xi^{7}+4 A^{2} B^{2} v_{\mathrm{r} y}^{2} \xi^{7}\right.$ $+8 A^{2} C^{2} v_{\mathrm{r} y}^{2} \xi^{7}+4 B^{2} C^{2} v_{\mathrm{r} y}^{2} \xi^{7}+4 A^{4} v_{\mathrm{r} z}^{2} \xi^{7}+4 B^{4} v_{\mathrm{r} z}^{2} \xi^{7}+8 A^{2} B^{2} v_{\mathrm{r} z}^{2} \xi^{7}+4 A^{2} C^{2} v_{\mathrm{r} z}^{2} \xi^{7}+4 B^{2} C^{2} v_{\mathrm{r} z}^{2} \xi^{7}-4 B^{4} v_{\mathrm{t} x}^{2} \xi^{7}$ $-4 C^{4} v_{\mathrm{t} x}^{2} \xi^{7}-4 A^{2} B^{2} v_{\mathrm{t} x}^{2} \xi^{7}-4 A^{2} C^{2} v_{\mathrm{t} x}^{2} \xi^{7}-8 B^{2} C^{2} v_{\mathrm{t} x}^{2} \xi^{7}-4 A^{4} v_{\mathrm{t} y}^{2} \xi^{7}-4 C^{4} v_{\mathrm{t} y}^{2} \xi^{7}-4 A^{2} B^{2} v_{\mathrm{t} y}^{2} \xi^{7}-8 A^{2} C^{2} v_{\mathrm{t} y}^{2} \xi^{7}$ $-4 B^{2} C^{2} v_{\mathrm{t} y}^{2} \xi^{7}-4 A^{4} v_{\mathrm{t} z}^{2} \xi^{7}-4 B^{4} v_{\mathrm{t} z}^{2} \xi^{7}-8 A^{2} B^{2} v_{\mathrm{t} z}^{2} \xi^{7}-4 A^{2} C^{2} v_{\mathrm{t} z}^{2} \xi^{7}-4 B^{2} C^{2} v_{\mathrm{t} z}^{2} \xi^{7}-8 A B^{3} v_{\mathrm{r} x} v_{\mathrm{r} y} \xi^{7}-8 A B C^{2} v_{\mathrm{r} x} v_{\mathrm{r} y} \xi^{7}$ $-8 A^{3} B v_{\mathrm{r} x} v_{\mathrm{r} y} \xi^{7}-8 A C^{3} v_{\mathrm{r} x} v_{\mathrm{r} z} \xi^{7}-8 A^{3} C v_{\mathrm{r} x} v_{\mathrm{r} z} \xi^{7}-8 A B^{2} C v_{\mathrm{r} x} v_{\mathrm{r} z} \xi^{7}-8 B C^{3} v_{\mathrm{r} y} v_{\mathrm{r} z} \xi^{7}-8 B^{3} C v_{\mathrm{r} y} v_{\mathrm{r} z} \xi^{7}$ $-8 A^{2} B C v_{\mathrm{r} y} v_{\mathrm{r} z} \xi^{7}+8 A B^{3} v_{\mathrm{t} x} v_{\mathrm{t} y} \xi^{7}+8 A B C^{2} v_{\mathrm{t} x} v_{\mathrm{t} y} \xi^{7}+8 A^{3} B v_{\mathrm{t} x} v_{\mathrm{t} y} \xi^{7}+8 A C^{3} v_{\mathrm{t} x} v_{\mathrm{t} z} \xi^{7}+8 A^{3} C v_{\mathrm{t} x} v_{\mathrm{t} z} \xi^{7}$ $+8 A B^{2} C v_{\mathrm{t} x} v_{\mathrm{t} z} \xi^{7}+8 B C^{3} v_{\mathrm{t} y} v_{\mathrm{t} z} \xi^{7}+8 B^{3} C v_{\mathrm{t} y} v_{\mathrm{t} z} \xi^{7}+8 A^{2} B C v_{\mathrm{t} y} v_{\mathrm{t} z} \xi^{7}-10 B^{4} v_{\mathrm{r} x}^{2} \xi^{5}-10 D C^{3} v_{\mathrm{r} x}^{2} \xi^{5}-10 A^{2} B^{2} v_{\mathrm{r} x}^{2} \xi^{5}$ $-4 A^{2} C^{2} v_{\mathrm{r} x}^{2} \xi^{5}-10 B^{2} C^{2} v_{\mathrm{r} x}^{2} \xi^{5}-8 A^{2} D C v_{\mathrm{r} x}^{2} \xi^{5}-10 B^{2} D C v_{\mathrm{r} x}^{2} \xi^{5}-10 A^{4} v_{\mathrm{r} y}^{2} \xi^{5}-10 D C^{3} v_{\mathrm{r} y}^{2} \xi^{5}-10 A^{2} B^{2} v_{\mathrm{r} y}^{2} \xi^{5}$ $-10 A^{2} C^{2} v_{\mathrm{r} y}^{2} \xi^{5}-4 B^{2} C^{2} v_{\mathrm{r} y}^{2} \xi^{5}-10 A^{2} D C v_{\mathrm{r} y}^{2} \xi^{5}-8 B^{2} D C v_{\mathrm{r} y}^{2} \xi^{5}-12 A^{4} v_{\mathrm{r} z}^{2} \xi^{5}-12 B^{4} v_{\mathrm{r} z}^{2} \xi^{5}-24 A^{2} B^{2} v_{\mathrm{r} z}^{2} \xi^{5}$ $-8 A^{2} C^{2} v_{\mathrm{r} z}^{2} \xi^{5}-8 B^{2} C^{2} v_{\mathrm{r} z}^{2} \xi^{5}-2 A^{2} D C v_{\mathrm{r} z}^{2} \xi^{5}-2 B^{2} D C v_{\mathrm{r} z}^{2} \xi^{5}+10 B^{4} v_{\mathrm{t} x}^{2} \xi^{5}-10 D C^{3} v_{\mathrm{t} x}^{2} \xi^{5}+10 A^{2} B^{2} v_{\mathrm{t} x}^{2} \xi^{5}+4 A^{2} C^{2} v_{\mathrm{t} x}^{2} \xi^{5}$ $+10 B^{2} C^{2} v_{\mathrm{t} x}^{2} \xi^{5}-8 A^{2} D C v_{\mathrm{t} x}^{2} \xi^{5}-10 B^{2} D C v_{\mathrm{t} x}^{2} \xi^{5}+10 A^{4} v_{\mathrm{t} y}^{2} \xi^{5}-10 D C^{3} v_{\mathrm{t} y}^{2} \xi^{5}+10 A^{2} B^{2} v_{\mathrm{t} y}^{2} \xi^{5}+10 A^{2} C^{2} v_{\mathrm{t} y}^{2} \xi^{5}$ $+4 B^{2} C^{2} v_{\mathrm{t} y}^{2} \xi^{5}-10 A^{2} D C v_{\mathrm{t} y}^{2} \xi^{5}-8 B^{2} D C v_{\mathrm{t} y}^{2} \xi^{5}+12 A^{4} v_{\mathrm{t} z}^{2} \xi^{5}+12 B^{4} v_{\mathrm{t} z}^{2} \xi^{5}+24 A^{2} B^{2} v_{\mathrm{t} z}^{2} \xi^{5}+8 A^{2} C^{2} v_{\mathrm{t} z}^{2} \xi^{5}+8 B^{2} C^{2} v_{\mathrm{t} z}^{2} \xi^{5}$ $-2 A^{2} D C v_{\mathrm{t} z}^{2} \xi^{5}-2 B^{2} D C v_{\mathrm{t} z}^{2} \xi^{5}+20 A B^{3} v_{\mathrm{r} x} v_{\mathrm{r} y} \xi^{5}+12 A B C^{2} v_{\mathrm{r} x} v_{\mathrm{r} y} \xi^{5}+20 A^{3} B v_{\mathrm{r} x} v_{\mathrm{r} y} \xi^{5}+4 A B D C v_{\mathrm{r} x} v_{\mathrm{r} y} \xi^{5}$ $+8 A C^{3} v_{\mathrm{r} x} v_{\mathrm{r} z} \xi^{5}+12 A D C^{2} v_{\mathrm{r} x} v_{\mathrm{r} z} \xi^{5}+8 A^{3} D v_{\mathrm{r} x} v_{\mathrm{r} z} \xi^{5}+8 A B^{2} D v_{\mathrm{r} x} v_{\mathrm{r} z} \xi^{5}+16 A^{3} C v_{\mathrm{r} x} v_{\mathrm{r} z} \xi^{5}+16 A B^{2} C v_{\mathrm{r} x} v_{\mathrm{r} z} \xi^{5}$ $+8 B C^{3} v_{\mathrm{r} y} v_{\mathrm{r} z} \xi^{5}+12 B D C^{2} v_{\mathrm{r} y} v_{\mathrm{r} z} \xi^{5}+8 B^{3} D v_{\mathrm{r} y} v_{\mathrm{r} z} \xi^{5}+8 A^{2} B D v_{\mathrm{r} y} v_{\mathrm{r} z} \xi^{5}+16 B^{3} C v_{\mathrm{r} y} v_{\mathrm{r} z} \xi^{5}+16 A^{2} B C v_{\mathrm{r} y} v_{\mathrm{r} z} \xi^{5}$ $-4 D C^{3} v_{\mathrm{r} x} v_{\mathrm{t} x} \xi^{5}-4 B^{2} D C v_{\mathrm{r} x} v_{\mathrm{t} x} \xi^{5}+4 A B D C v_{\mathrm{r} y} v_{\mathrm{t} x} \xi^{5}+4 A D C^{2} v_{\mathrm{r} z} v_{\mathrm{t} x} \xi^{5}+4 A B D C v_{\mathrm{r} x} v_{\mathrm{t} y} \xi^{5}-4 D C^{3} v_{\mathrm{r} y} v_{\mathrm{t} y} \xi^{5}$ $-4 A^{2} D C v_{\mathrm{r} y} v_{\mathrm{t} y} \xi^{5}+4 B D C^{2} v_{\mathrm{r} z} v_{\mathrm{t} y} \xi^{5}-20 A B^{3} v_{\mathrm{t} x} v_{\mathrm{t} y} \xi^{5}-12 A B C^{2} v_{\mathrm{t} x} v_{\mathrm{t} y} \xi^{5}-20 A^{3} B v_{\mathrm{t} x} v_{\mathrm{t} y} \xi^{5}+4 A B D C v_{\mathrm{t} x} v_{\mathrm{t} y} \xi^{5}$ $+4 A D C^{2} v_{\mathrm{r} x} v_{\mathrm{t} z} \xi^{5}+4 B D C^{2} v_{\mathrm{r} y} v_{\mathrm{t} z} \xi^{5}-4 A^{2} D C v_{\mathrm{r} z} v_{\mathrm{t} z} \xi^{5}-4 B^{2} D C v_{\mathrm{r} z} v_{\mathrm{t} z} \xi^{5}-8 A C^{3} v_{\mathrm{t} x} v_{\mathrm{t} z} \xi^{5}+12 A D C^{2} v_{\mathrm{t} x} v_{\mathrm{t} z} \xi^{5}$ $+8 A^{3} D v_{\mathrm{t} x} v_{\mathrm{t} z} \xi^{5}+8 A B^{2} D v_{\mathrm{t} x} v_{\mathrm{t} z} \xi^{5}-16 A^{3} C v_{\mathrm{t} x} v_{\mathrm{t} z} \xi^{5}-16 A B^{2} C v_{\mathrm{t} x} v_{\mathrm{t} z} \xi^{5}-8 B C^{3} v_{\mathrm{t} y} v_{\mathrm{t} z} \xi^{5}+12 B D C^{2} v_{\mathrm{t} y} v_{\mathrm{t} z} \xi^{5}$ $+8 B^{3} D v_{\mathrm{t} y} v_{\mathrm{t} z} \xi^{5}+8 A^{2} B D v_{\mathrm{t} y} v_{\mathrm{t} z} \xi^{5}-16 B^{3} C v_{\mathrm{t} y} v_{\mathrm{t} z} \xi^{5}-16 A^{2} B C v_{\mathrm{t} y} v_{\mathrm{t} z} \xi^{5}+8 B^{4} v_{\mathrm{r} x}^{2} \xi^{3}+8 A^{2} B^{2} v_{\mathrm{r} x}^{2} \xi^{3}+4 A^{2} D^{2} v_{\mathrm{r} x}^{2} \xi^{3}$ $+2 B^{2} D^{2} v_{\mathrm{r} x}^{2} \xi^{3}+2 B^{2} C^{2} v_{\mathrm{r} x}^{2} \xi^{3}+8 D^{2} C^{2} v_{\mathrm{r} x}^{2} \xi^{3}+8 A^{2} D C v_{\mathrm{r} x}^{2} \xi^{3}+12 B^{2} D C v_{\mathrm{r} x}^{2} \xi^{3}+8 A^{4} v_{\mathrm{r} y}^{2} \xi^{3}+8 A^{2} B^{2} v_{\mathrm{r} y}^{2} \xi^{3}+2 A^{2} D^{2} v_{\mathrm{r} y}^{2} \xi^{3}$ $+4 B^{2} D^{2} v_{\mathrm{r} y}^{2} \xi^{3}+2 A^{2} C^{2} v_{\mathrm{r} y}^{2} \xi^{3}+8 D^{2} C^{2} v_{\mathrm{r} y}^{2} \xi^{3}+12 A^{2} D C v_{\mathrm{r} y}^{2} \xi^{3}+8 B^{2} D C v_{\mathrm{r} y}^{2} \xi^{3}+12 A^{4} v_{\mathrm{r} z}^{2} \xi^{3}+12 B^{4} v_{\mathrm{r} z}^{2} \xi^{3}+24 A^{2} B^{2} v_{\mathrm{r} z}^{2} \xi^{3}$ $+4 A^{2} C^{2} v_{\mathrm{r} z}^{2} \xi^{3}+4 B^{2} C^{2} v_{\mathrm{r} z}^{2} \xi^{3}+4 A^{2} D C v_{\mathrm{r} z}^{2} \xi^{3}+4 B^{2} D C v_{\mathrm{r} z}^{2} \xi^{3}-8 B^{4} v_{\mathrm{t} x}^{2} \xi^{3}-8 A^{2} B^{2} v_{\mathrm{t} x}^{2} \xi^{3}-4 A^{2} D^{2} v_{\mathrm{t} x}^{2} \xi^{3}-2 B^{2} D^{2} v_{\mathrm{t} x}^{2} \xi^{3}$ $-2 B^{2} C^{2} v_{\mathrm{t} x}^{2} \xi^{3}-8 D^{2} C^{2} v_{\mathrm{t} x}^{2} \xi^{3}+8 A^{2} D C v_{\mathrm{t} x}^{2} \xi^{3}+12 B^{2} D C v_{\mathrm{t} x}^{2} \xi^{3}-8 A^{4} v_{\mathrm{t} y}^{2} \xi^{3}-8 A^{2} B^{2} v_{\mathrm{t} y}^{2} \xi^{3}-2 A^{2} D^{2} v_{\mathrm{t} y}^{2} \xi^{3}-4 B^{2} D^{2} v_{\mathrm{t} y}^{2} \xi^{3}$ $-2 A^{2} C^{2} v_{\mathrm{t} y}^{2} \xi^{3}-8 D^{2} C^{2} v_{\mathrm{t} y}^{2} \xi^{3}+12 A^{2} D C v_{\mathrm{t} y}^{2} \xi^{3}+8 B^{2} D C v_{\mathrm{t} y}^{2} \xi^{3}-12 A^{4} v_{\mathrm{t} z}^{2} \xi^{3}-12 B^{4} v_{\mathrm{t} z}^{2} \xi^{3}-24 A^{2} B^{2} v_{\mathrm{t} z}^{2} \xi^{3}-4 A^{2} C^{2} v_{\mathrm{t} z}^{2} \xi^{3}$ $-4 B^{2} C^{2} v_{\mathrm{t} z}^{2} \xi^{3}+4 A^{2} D C v_{\mathrm{t} z}^{2} \xi^{3}+4 B^{2} D C v_{\mathrm{t} z}^{2} \xi^{3}-16 A B^{3} v_{\mathrm{r} x} v_{\mathrm{r} y} \xi^{3}+4 A B D^{2} v_{\mathrm{r} x} v_{\mathrm{r} y} \xi^{3}-4 A B C^{2} v_{\mathrm{r} x} v_{\mathrm{r} y} \xi^{3}-16 A^{3} B v_{\mathrm{r} x} v_{\mathrm{r} y} \xi^{3}$ $-8 A B D C v_{\mathrm{r} x} v_{\mathrm{r} y} \xi^{3}-12 A D C^{2} v_{\mathrm{r} x} v_{\mathrm{r} z} \xi^{3}-16 A^{3} D v_{\mathrm{r} x} v_{\mathrm{r} z} \xi^{3}-16 A B^{2} D v_{\mathrm{r} x} v_{\mathrm{r} z} \xi^{3}-8 A^{3} C v_{\mathrm{r} x} v_{\mathrm{r} z} \xi^{3}-8 A B^{2} C v_{\mathrm{r} x} v_{\mathrm{r} z} \xi^{3}$ $-4 A D^{2} C v_{\mathrm{r} x} v_{\mathrm{r} z} \xi^{3}-12 B D C^{2} v_{\mathrm{r} y} v_{\mathrm{r} z} \xi^{3}-16 B^{3} D v_{\mathrm{r} y} v_{\mathrm{r} z} \xi^{3}-16 A^{2} B D v_{\mathrm{r} y} v_{\mathrm{r} z} \xi^{3}-8 B^{3} C v_{\mathrm{r} y} v_{\mathrm{r} z} \xi^{3}-4 B D^{2} C v_{\mathrm{r} y} v_{\mathrm{r} z} \xi^{3}$ $-8 A^{2} B C v_{\mathrm{r} y} v_{\mathrm{r} z} \xi^{3}-4 A D C^{2} v_{\mathrm{r} z} v_{\mathrm{t} x} \xi^{3}+4 A D^{2} C v_{\mathrm{r} z} v_{\mathrm{t} x} \xi^{3}-4 B D C^{2} v_{\mathrm{r} z} v_{\mathrm{t} y} \xi^{3}+4 B D^{2} C v_{\mathrm{r} z} v_{\mathrm{t} y} \xi^{3}+16 A B^{3} v_{\mathrm{t} x} v_{\mathrm{t} y} \xi^{3}$ $-4 A B D^{2} v_{\mathrm{t} x} v_{\mathrm{t} y} \xi^{3}+4 A B C^{2} v_{\mathrm{t} x} v_{\mathrm{t} y} \xi^{3}+16 A^{3} B v_{\mathrm{t} x} v_{\mathrm{t} y} \xi^{3}-8 A B D C v_{\mathrm{t} x} v_{\mathrm{t} y} \xi^{3}-4 A D C^{2} v_{\mathrm{r} x} v_{\mathrm{t} z} \xi^{3}-4 A D^{2} C v_{\mathrm{r} x} v_{\mathrm{t} z} \xi^{3}$ $-4 B D C^{2} v_{\mathrm{r} y} v_{\mathrm{t} z} \xi^{3}-4 B D^{2} C v_{\mathrm{r} y} v_{\mathrm{t} z} \xi^{3}+8 A^{2} D C v_{\mathrm{r} z} v_{\mathrm{t} z} \xi^{3}+8 B^{2} D C v_{\mathrm{r} z} v_{\mathrm{t} z} \xi^{3}-12 A D C^{2} v_{\mathrm{t} x} v_{\mathrm{t} z} \xi^{3}-16 A^{3} D v_{\mathrm{t} x} v_{\mathrm{t} z} \xi^{3}$ $-16 A B^{2} D v_{\mathrm{t} x} v_{\mathrm{t} z} \xi^{3}+8 A^{3} C v_{\mathrm{t} x} v_{\mathrm{t} z} \xi^{3}+8 A B^{2} C v_{\mathrm{t} x} v_{\mathrm{t} z} \xi^{3}+4 A D^{2} C v_{\mathrm{t} x} v_{\mathrm{t} z} \xi^{3}-12 B D C^{2} v_{\mathrm{t} y} v_{\mathrm{t} z} \xi^{3}-16 B^{3} D v_{\mathrm{t} y} v_{\mathrm{t} z} \xi^{3}$ $-16 A^{2} B D v_{\mathrm{t} y} v_{\mathrm{t} z} \xi^{3}+8 B^{3} C v_{\mathrm{t} y} v_{\mathrm{t} z} \xi^{3}+4 B D^{2} C v_{\mathrm{t} y} v_{\mathrm{t} z} \xi^{3}+8 A^{2} B C v_{\mathrm{t} y} v_{\mathrm{t} z} \xi^{3}-2 B^{4} v_{\mathrm{r} x}^{2} \xi-2 A^{2} B^{2} v_{\mathrm{r} x}^{2} \xi-4 A^{2} D^{2} v_{\mathrm{r} x}^{2} \xi$ $-2 B^{2} D^{2} v_{\mathrm{r} x}^{2} \xi-2 D^{3} C v_{\mathrm{r} x}^{2} \xi-2 B^{2} D C v_{\mathrm{r} x}^{2} \xi-2 A^{4} v_{\mathrm{r} y}^{2} \xi-2 A^{2} B^{2} v_{\mathrm{r} y}^{2} \xi-2 A^{2} D^{2} v_{\mathrm{r} y}^{2} \xi-4 B^{2} D^{2} v_{\mathrm{r} y}^{2} \xi-2 D^{3} C v_{\mathrm{r} y}^{2} \xi-2 A^{2} D C v_{\mathrm{r} y}^{2} \xi$ $-4 A^{4} v_{\mathrm{r} z}^{2} \xi-4 B^{4} v_{\mathrm{r} z}^{2} \xi-8 A^{2} B^{2} v_{\mathrm{r} z}^{2} \xi-2 A^{2} D C v_{\mathrm{r} z}^{2} \xi-2 B^{2} D C v_{\mathrm{r} z}^{2} \xi+2 B^{4} v_{\mathrm{t} x}^{2} \xi+2 A^{2} B^{2} v_{\mathrm{t} x}^{2} \xi+4 A^{2} D^{2} v_{\mathrm{t} x}^{2} \xi+2 B^{2} D^{2} v_{\mathrm{t} x}^{2} \xi$ $-2 D^{3} C v_{\mathrm{t} x}^{2} \xi-2 B^{2} D C v_{\mathrm{t} x}^{2} \xi+2 A^{4} v_{\mathrm{t} y}^{2} \xi+2 A^{2} B^{2} v_{\mathrm{t} y}^{2} \xi+2 A^{2} D^{2} v_{\mathrm{t} y}^{2} \xi+4 B^{2} D^{2} v_{\mathrm{t} y}^{2} \xi-2 D^{3} C v_{\mathrm{t} y}^{2} \xi-2 A^{2} D C v_{\mathrm{t} y}^{2} \xi+4 A^{4} v_{\mathrm{t} z}^{2} \xi$ $+4 B^{4} v_{\mathrm{t} z}^{2} \xi+8 A^{2} B^{2} v_{\mathrm{t} z}^{2} \xi-2 A^{2} D C v_{\mathrm{t} z}^{2} \xi-2 B^{2} D C v_{\mathrm{t} z}^{2} \xi+4 A B^{3} v_{\mathrm{r} x} v_{\mathrm{r} y} \xi-4 A B D^{2} v_{\mathrm{r} x} v_{\mathrm{r} y} \xi+4 A^{3} B v_{\mathrm{r} x} v_{\mathrm{r} y} \xi+4 A B D C v_{\mathrm{r} x} v_{\mathrm{r} y} \xi$ $+8 A^{3} D v_{\mathrm{r} x} v_{\mathrm{r} z} \xi+8 A B^{2} D v_{\mathrm{r} x} v_{\mathrm{r} z} \xi+4 A D^{2} C v_{\mathrm{r} x} v_{\mathrm{r} z} \xi+8 B^{3} D v_{\mathrm{r} y} v_{\mathrm{r} z} \xi+8 A^{2} B D v_{\mathrm{r} y} v_{\mathrm{r} z} \xi+4 B D^{2} C v_{\mathrm{r} y} v_{\mathrm{r} z} \xi+4 D^{3} C v_{\mathrm{r} x} v_{\mathrm{t} x} \xi$ $+4 B^{2} D C v_{\mathrm{r} x} v_{\mathrm{t} x} \xi-4 A B D C v_{\mathrm{r} y} v_{\mathrm{t} x} \xi-4 A D^{2} C v_{\mathrm{r} z} v_{\mathrm{t} x} \xi-4 A B D C v_{\mathrm{r} x} v_{\mathrm{t} y} \xi+4 D^{3} C v_{\mathrm{r} y} v_{\mathrm{t} y} \xi+4 A^{2} D C v_{\mathrm{r} y} v_{\mathrm{t} y} \xi-4 B D^{2} C v_{\mathrm{r} z} v_{\mathrm{t} y} \xi$ $-4 A B^{3} v_{\mathrm{t} x} v_{\mathrm{t} y} \xi+4 A B D^{2} v_{\mathrm{t} x} v_{\mathrm{t} y} \xi-4 A^{3} B v_{\mathrm{t} x} v_{\mathrm{t} y} \xi+4 A B D C v_{\mathrm{t} x} v_{\mathrm{t} y} \xi+4 A D^{2} C v_{\mathrm{r} x} v_{\mathrm{t} z} \xi+4 B D^{2} C v_{\mathrm{r} y} v_{\mathrm{t} z} \xi-4 A^{2} D C v_{\mathrm{r} z} v_{\mathrm{t} z} \xi$ $-4 B^{2} D C v_{\mathrm{r} z} v_{\mathrm{t} z} \xi+8 A^{3} D v_{\mathrm{t} x} v_{\mathrm{t} z} \xi+8 A B^{2} D v_{\mathrm{t} x} v_{\mathrm{t} z} \xi-4 A D^{2} C v_{\mathrm{t} x} v_{\mathrm{t} z} \xi+8 B^{3} D v_{\mathrm{t} y} v_{\mathrm{t} z} \xi+8 A^{2} B D v_{\mathrm{t} y} v_{\mathrm{t} z} \xi$ $\left.-4 B D^{2} C v_{\mathrm{t} y} v_{\mathrm{t} z} \xi\right) \eta^{5}$ 
$+\left(6 B^{4} v_{\mathrm{r} x}^{2} \xi^{8}+6 C^{4} v_{\mathrm{r} x}^{2} \xi^{8}+6 A^{2} B^{2} v_{\mathrm{r} x}^{2} \xi^{8}+6 A^{2} C^{2} v_{\mathrm{r} x}^{2} \xi^{8}+12 B^{2} C^{2} v_{\mathrm{r} x}^{2} \xi^{8}+6 A^{4} v_{\mathrm{r} y}^{2} \xi^{8}+6 C^{4} v_{\mathrm{r} y}^{2} \xi^{8}+6 A^{2} B^{2} v_{\mathrm{r} y}^{2} \xi^{8}\right.$ $+12 A^{2} C^{2} v_{\mathrm{r} y}^{2} \xi^{8}+6 B^{2} C^{2} v_{\mathrm{r} y}^{2} \xi^{8}+6 A^{4} v_{\mathrm{r} z}^{2} \xi^{8}+6 B^{4} v_{\mathrm{r} z}^{2} \xi^{8}+12 A^{2} B^{2} v_{\mathrm{r} z}^{2} \xi^{8}+6 A^{2} C^{2} v_{\mathrm{r} z}^{2} \xi^{8}+6 B^{2} C^{2} v_{\mathrm{r} z}^{2} \xi^{8}+6 B^{4} v_{\mathrm{t} x}^{2} \xi^{8}$ $+6 C^{4} v_{\mathrm{t} x}^{2} \xi^{8}+6 A^{2} B^{2} v_{\mathrm{t} x}^{2} \xi^{8}+6 A^{2} C^{2} v_{\mathrm{t} x}^{2} \xi^{8}+12 B^{2} C^{2} v_{\mathrm{t} x}^{2} \xi^{8}+6 A^{4} v_{\mathrm{t} y}^{2} \xi^{8}+6 C^{4} v_{\mathrm{t} y}^{2} \xi^{8}+6 A^{2} B^{2} v_{\mathrm{t} y}^{2} \xi^{8}+12 A^{2} C^{2} v_{\mathrm{t} y}^{2} \xi^{8}$ $+6 B^{2} C^{2} v_{\mathrm{t} y}^{2} \xi^{8}+6 A^{4} v_{\mathrm{t} z}^{2} \xi^{8}+6 B^{4} v_{\mathrm{t} z}^{2} \xi^{8}+12 A^{2} B^{2} v_{\mathrm{t} z}^{2} \xi^{8}+6 A^{2} C^{2} v_{\mathrm{t} z}^{2} \xi^{8}+6 B^{2} C^{2} v_{\mathrm{t} z}^{2} \xi^{8}-12 A B^{3} v_{\mathrm{r} x} v_{\mathrm{r} y} \xi^{8}$ $-12 A B C^{2} v_{\mathrm{r} x} v_{\mathrm{r} y} \xi^{8}-12 A^{3} B v_{\mathrm{r} x} v_{\mathrm{r} y} \xi^{8}-12 A C^{3} v_{\mathrm{r} x} v_{\mathrm{r} z} \xi^{8}-12 A^{3} C v_{\mathrm{r} x} v_{\mathrm{r} z} \xi^{8}-12 A B^{2} C v_{\mathrm{r} x} v_{\mathrm{r} z} \xi^{8}-12 B C^{3} v_{\mathrm{r} y} v_{\mathrm{r} z} \xi^{8}$ $-12 B^{3} C v_{\mathrm{r} y} v_{\mathrm{r} z} \xi^{8}-12 A^{2} B C v_{\mathrm{r} y} v_{\mathrm{r} z} \xi^{8}-4 B^{4} v_{\mathrm{r} x} v_{\mathrm{t} x} \xi^{8}-4 C^{4} v_{\mathrm{r} x} v_{\mathrm{t} x} \xi^{8}-4 A^{2} B^{2} v_{\mathrm{r} x} v_{\mathrm{t} x} \xi^{8}-4 A^{2} C^{2} v_{\mathrm{r} x} v_{\mathrm{t} x} \xi^{8}$ $-8 B^{2} C^{2} v_{\mathrm{r} x} v_{\mathrm{t} x} \xi^{8}+4 A B^{3} v_{\mathrm{r} y} v_{\mathrm{t} x} \xi^{8}+4 A B C^{2} v_{\mathrm{r} y} v_{\mathrm{t} x} \xi^{8}+4 A^{3} B v_{\mathrm{r} y} v_{\mathrm{t} x} \xi^{8}+4 A C^{3} v_{\mathrm{r} z} v_{\mathrm{t} x} \xi^{8}+4 A^{3} C v_{\mathrm{r} z} v_{\mathrm{t} x} \xi^{8}$ $+4 A B^{2} C v_{\mathrm{r} z} v_{\mathrm{t} x} \xi^{8}+4 A B^{3} v_{\mathrm{r} x} v_{\mathrm{t} y} \xi^{8}+4 A B C^{2} v_{\mathrm{r} x} v_{\mathrm{t} y} \xi^{8}+4 A^{3} B v_{\mathrm{r} x} v_{\mathrm{t} y} \xi^{8}-4 A^{4} v_{\mathrm{r} y} v_{\mathrm{t} y} \xi^{8}-4 C^{4} v_{\mathrm{r} y} v_{\mathrm{t} y} \xi^{8}-4 A^{2} B^{2} v_{\mathrm{r} y} v_{\mathrm{t} y} \xi^{8}$ $-8 A^{2} C^{2} v_{\mathrm{r} y} v_{\mathrm{t} y} \xi^{8}-4 B^{2} C^{2} v_{\mathrm{r} y} v_{\mathrm{t} y} \xi^{8}+4 B C^{3} v_{\mathrm{r} z} v_{\mathrm{t} y} \xi^{8}+4 B^{3} C v_{\mathrm{r} z} v_{\mathrm{t} y} \xi^{8}+4 A^{2} B C v_{\mathrm{r} z} v_{\mathrm{t} y} \xi^{8}-12 A B^{3} v_{\mathrm{t} x} v_{\mathrm{t} y} \xi^{8}$ $-12 A B C^{2} v_{\mathrm{t} x} v_{\mathrm{t} y} \xi^{8}-12 A^{3} B v_{\mathrm{t} x} v_{\mathrm{t} y} \xi^{8}+4 A C^{3} v_{\mathrm{r} x} v_{\mathrm{t} z} \xi^{8}+4 A^{3} C v_{\mathrm{r} x} v_{\mathrm{t} z} \xi^{8}+4 A B^{2} C v_{\mathrm{r} x} v_{\mathrm{t} z} \xi^{8}+4 B C^{3} v_{\mathrm{r} y} v_{\mathrm{t} z} \xi^{8}$ $+4 B^{3} C v_{\mathrm{r} y} v_{\mathrm{t} z} \xi^{8}+4 A^{2} B C v_{\mathrm{r} y} v_{\mathrm{t} z} \xi^{8}-4 A^{4} v_{\mathrm{r} z} v_{\mathrm{t} z} \xi^{8}-4 B^{4} v_{\mathrm{r} z} v_{\mathrm{t} z} \xi^{8}-8 A^{2} B^{2} v_{\mathrm{r} z} v_{\mathrm{t} z} \xi^{8}-4 A^{2} C^{2} v_{\mathrm{r} z} v_{\mathrm{t} z} \xi^{8}-4 B^{2} C^{2} v_{\mathrm{r} z} v_{\mathrm{t} z} \xi^{8}$ $-12 A C^{3} v_{\mathrm{t} x} v_{\mathrm{t} z} \xi^{8}-12 A^{3} C v_{\mathrm{t} x} v_{\mathrm{t} z} \xi^{8}-12 A B^{2} C v_{\mathrm{t} x} v_{\mathrm{t} z} \xi^{8}-12 B C^{3} v_{\mathrm{t} y} v_{\mathrm{t} z} \xi^{8}-12 B^{3} C v_{\mathrm{t} y} v_{\mathrm{t} z} \xi^{8}-12 A^{2} B C v_{\mathrm{t} y} v_{\mathrm{t} z} \xi^{8}$ $-20 B^{4} v_{\mathrm{r} x}^{2} \xi^{6}-20 D C^{3} v_{\mathrm{r} x}^{2} \xi^{6}-20 A^{2} B^{2} v_{\mathrm{r} x}^{2} \xi^{6}-7 A^{2} C^{2} v_{\mathrm{r} x}^{2} \xi^{6}-20 B^{2} C^{2} v_{\mathrm{r} x}^{2} \xi^{6}-12 A^{2} D C v_{\mathrm{r} x}^{2} \xi^{6}-20 B^{2} D C v_{\mathrm{r} x}^{2} \xi^{6}$ $-20 A^{4} v_{\mathrm{r} y}^{2} \xi^{6}-20 D C^{3} v_{\mathrm{r} y}^{2} \xi^{6}-20 A^{2} B^{2} v_{\mathrm{r} y}^{2} \xi^{6}-20 A^{2} C^{2} v_{\mathrm{r} y}^{2} \xi^{6}-7 B^{2} C^{2} v_{\mathrm{r} y}^{2} \xi^{6}-20 A^{2} D C v_{\mathrm{r} y}^{2} \xi^{6}-12 B^{2} D C v_{\mathrm{r} y}^{2} \xi^{6}$ $-19 A^{4} v_{\mathrm{r} z}^{2} \xi^{6}-19 B^{4} v_{\mathrm{r} z}^{2} \xi^{6}-38 A^{2} B^{2} v_{\mathrm{r} z}^{2} \xi^{6}-12 A^{2} C^{2} v_{\mathrm{r} z}^{2} \xi^{6}-12 B^{2} C^{2} v_{\mathrm{r} z}^{2} \xi^{6}-8 A^{2} D C v_{\mathrm{r} z}^{2} \xi^{6}-8 B^{2} D C v_{\mathrm{r} z}^{2} \xi^{6}-20 B^{4} v_{\mathrm{t} x}^{2} \xi^{6}$ $+20 D C^{3} v_{\mathrm{t} x}^{2} \xi^{6}-20 A^{2} B^{2} v_{\mathrm{t} x}^{2} \xi^{6}-7 A^{2} C^{2} v_{\mathrm{t} x}^{2} \xi^{6}-20 B^{2} C^{2} v_{\mathrm{t} x}^{2} \xi^{6}+12 A^{2} D C v_{\mathrm{t} x}^{2} \xi^{6}+20 B^{2} D C v_{\mathrm{t} x}^{2} \xi^{6}-20 A^{4} v_{\mathrm{t} y}^{2} \xi^{6}$ $+20 D C^{3} v_{\mathrm{t} y}^{2} \xi^{6}-20 A^{2} B^{2} v_{\mathrm{t} y}^{2} \xi^{6}-20 A^{2} C^{2} v_{\mathrm{t} y}^{2} \xi^{6}-7 B^{2} C^{2} v_{\mathrm{t} y}^{2} \xi^{6}+20 A^{2} D C v_{\mathrm{t} y}^{2} \xi^{6}+12 B^{2} D C v_{\mathrm{t} y}^{2} \xi^{6}-19 A^{4} v_{\mathrm{t} z}^{2} \xi^{6}$ $-19 B^{4} v_{\mathrm{t} z}^{2} \xi^{6}-38 A^{2} B^{2} v_{\mathrm{t} z}^{2} \xi^{6}-12 A^{2} C^{2} v_{\mathrm{t} z}^{2} \xi^{6}-12 B^{2} C^{2} v_{\mathrm{t} z}^{2} \xi^{6}+8 A^{2} D C v_{\mathrm{t} z}^{2} \xi^{6}+8 B^{2} D C v_{\mathrm{t} z}^{2} \xi^{6}+40 A B^{3} v_{\mathrm{r} x} v_{\mathrm{r} y} \xi^{6}$ $+26 A B C^{2} v_{\mathrm{r} x} v_{\mathrm{r} y} \xi^{6}+40 A^{3} B v_{\mathrm{r} x} v_{\mathrm{r} y} \xi^{6}+16 A B D C v_{\mathrm{r} x} v_{\mathrm{r} y} \xi^{6}+12 A C^{3} v_{\mathrm{r} x} v_{\mathrm{r} z} \xi^{6}+28 A D C^{2} v_{\mathrm{r} x} v_{\mathrm{r} z} \xi^{6}+12 A^{3} D v_{\mathrm{r} x} v_{\mathrm{r} z} \xi^{6}$ $+12 A B^{2} D v_{\mathrm{r} x} v_{\mathrm{r} z} \xi^{6}+26 A^{3} C v_{\mathrm{r} x} v_{\mathrm{r} z} \xi^{6}+26 A B^{2} C v_{\mathrm{r} x} v_{\mathrm{r} z} \xi^{6}+12 B C^{3} v_{\mathrm{r} y} v_{\mathrm{r} z} \xi^{6}+28 B D C^{2} v_{\mathrm{r} y} v_{\mathrm{r} z} \xi^{6}+12 B^{3} D v_{\mathrm{r} y} v_{\mathrm{r} z} \xi^{6}$ $+12 A^{2} B D v_{\mathrm{r} y} v_{\mathrm{r} z} \xi^{6}+26 B^{3} C v_{\mathrm{r} y} v_{\mathrm{r} z} \xi^{6}+26 A^{2} B C v_{\mathrm{r} y} v_{\mathrm{r} z} \xi^{6}+8 B^{4} v_{\mathrm{r} x} v_{\mathrm{t} x} \xi^{6}+8 A^{2} B^{2} v_{\mathrm{r} x} v_{\mathrm{t} x} \xi^{6}+2 A^{2} C^{2} v_{\mathrm{r} x} v_{\mathrm{t} x} \xi^{6}$ $+8 B^{2} C^{2} v_{\mathrm{r} x} v_{\mathrm{t} x} \xi^{6}-8 A B^{3} v_{\mathrm{r} y} v_{\mathrm{t} x} \xi^{6}-6 A B C^{2} v_{\mathrm{r} y} v_{\mathrm{t} x} \xi^{6}-8 A^{3} B v_{\mathrm{r} y} v_{\mathrm{t} x} \xi^{6}-4 A C^{3} v_{\mathrm{r} z} v_{\mathrm{t} x} \xi^{6}+4 A D C^{2} v_{\mathrm{r} z} v_{\mathrm{t} x} \xi^{6}$ $+4 A^{3} D v_{\mathrm{r} z} v_{\mathrm{t} x} \xi^{6}+4 A B^{2} D v_{\mathrm{r} z} v_{\mathrm{t} x} \xi^{6}-6 A^{3} C v_{\mathrm{r} z} v_{\mathrm{t} x} \xi^{6}-6 A B^{2} C v_{\mathrm{r} z} v_{\mathrm{t} x} \xi^{6}-8 A B^{3} v_{\mathrm{r} x} v_{\mathrm{t} y} \xi^{6}-6 A B C^{2} v_{\mathrm{r} x} v_{\mathrm{t} y} \xi^{6}$ $-8 A^{3} B v_{\mathrm{r} x} v_{\mathrm{t} y} \xi^{6}+8 A^{4} v_{\mathrm{r} y} v_{\mathrm{t} y} \xi^{6}+8 A^{2} B^{2} v_{\mathrm{r} y} v_{\mathrm{t} y} \xi^{6}+8 A^{2} C^{2} v_{\mathrm{r} y} v_{\mathrm{t} y} \xi^{6}+2 B^{2} C^{2} v_{\mathrm{r} y} v_{\mathrm{t} y} \xi^{6}-4 B C^{3} v_{\mathrm{r} z} v_{\mathrm{t} y} \xi^{6}$ $+4 B D C^{2} v_{\mathrm{r} z} v_{\mathrm{t} y} \xi^{6}+4 B^{3} D v_{\mathrm{r} z} v_{\mathrm{t} y} \xi^{6}+4 A^{2} B D v_{\mathrm{r} z} v_{\mathrm{t} y} \xi^{6}-6 B^{3} C v_{\mathrm{r} z} v_{\mathrm{t} y} \xi^{6}-6 A^{2} B C v_{\mathrm{r} z} v_{\mathrm{t} y} \xi^{6}+40 A B^{3} v_{\mathrm{t} x} v_{\mathrm{t} y} \xi^{6}$ $+26 A B C^{2} v_{\mathrm{t} x} v_{\mathrm{t} y} \xi^{6}+40 A^{3} B v_{\mathrm{t} x} v_{\mathrm{t} y} \xi^{6}-16 A B D C v_{\mathrm{t} x} v_{\mathrm{t} y} \xi^{6}-4 A C^{3} v_{\mathrm{r} x} v_{\mathrm{t} z} \xi^{6}-4 A D C^{2} v_{\mathrm{r} x} v_{\mathrm{t} z} \xi^{6}-4 A^{3} D v_{\mathrm{r} x} v_{\mathrm{t} z} \xi^{6}$ $-4 A B^{2} D v_{\mathrm{r} x} v_{\mathrm{t} z} \xi^{6}-6 A^{3} C v_{\mathrm{r} x} v_{\mathrm{t} z} \xi^{6}-6 A B^{2} C v_{\mathrm{r} x} v_{\mathrm{t} z} \xi^{6}-4 B C^{3} v_{\mathrm{r} y} v_{\mathrm{t} z} \xi^{6}-4 B D C^{2} v_{\mathrm{r} y} v_{\mathrm{t} z} \xi^{6}-4 B^{3} D v_{\mathrm{r} y} v_{\mathrm{t} z} \xi^{6}$ $-4 A^{2} B D v_{\mathrm{r} y} v_{\mathrm{t} z} \xi^{6}-6 B^{3} C v_{\mathrm{r} y} v_{\mathrm{t} z} \xi^{6}-6 A^{2} B C v_{\mathrm{r} y} v_{\mathrm{t} z} \xi^{6}+10 A^{4} v_{\mathrm{r} z} v_{\mathrm{t} z} \xi^{6}+10 B^{4} v_{\mathrm{r} z} v_{\mathrm{t} z} \xi^{6}+20 A^{2} B^{2} v_{\mathrm{r} z} v_{\mathrm{t} z} \xi^{6}$ $+8 A^{2} C^{2} v_{\mathrm{r} z} v_{\mathrm{t} z} \xi^{6}+8 B^{2} C^{2} v_{\mathrm{r} z} v_{\mathrm{t} z} \xi^{6}+12 A C^{3} v_{\mathrm{t} x} v_{\mathrm{t} z} \xi^{6}-28 A D C^{2} v_{\mathrm{t} x} v_{\mathrm{t} z} \xi^{6}-12 A^{3} D v_{\mathrm{t} x} v_{\mathrm{t} z} \xi^{6}-12 A B^{2} D v_{\mathrm{t} x} v_{\mathrm{t} z} \xi^{6}$ $+26 A^{3} C v_{\mathrm{t} x} v_{\mathrm{t} z} \xi^{6}+26 A B^{2} C v_{\mathrm{t} x} v_{\mathrm{t} z} \xi^{6}+12 B C^{3} v_{\mathrm{t} y} v_{\mathrm{t} z} \xi^{6}-28 B D C^{2} v_{\mathrm{t} y} v_{\mathrm{t} z} \xi^{6}-12 B^{3} D v_{\mathrm{t} y} v_{\mathrm{t} z} \xi^{6}-12 A^{2} B D v_{\mathrm{t} y} v_{\mathrm{t} z} \xi^{6}$ $+26 B^{3} C v_{\mathrm{t} y} v_{\mathrm{t} z} \xi^{6}+26 A^{2} B C v_{\mathrm{t} y} v_{\mathrm{t} z} \xi^{6}+23 B^{4} v_{\mathrm{r} x}^{2} \xi^{4}+23 A^{2} B^{2} v_{\mathrm{r} x}^{2} \xi^{4}+6 A^{2} D^{2} v_{\mathrm{r} x}^{2} \xi^{4}+8 B^{2} D^{2} v_{\mathrm{r} x}^{2} \xi^{4}+A^{2} C^{2} v_{\mathrm{r} x}^{2} \xi^{4}$ $+8 B^{2} C^{2} v_{\mathrm{r} x}^{2} \xi^{4}+23 D^{2} C^{2} v_{\mathrm{r} x}^{2} \xi^{4}+14 A^{2} D C v_{\mathrm{r} x}^{2} \xi^{4}+30 B^{2} D C v_{\mathrm{r} x}^{2} \xi^{4}+23 A^{4} v_{\mathrm{r} y}^{2} \xi^{4}+23 A^{2} B^{2} v_{\mathrm{r} y}^{2} \xi^{4}+8 A^{2} D^{2} v_{\mathrm{r} y}^{2} \xi^{4}$ $+6 B^{2} D^{2} v_{\mathrm{r} y}^{2} \xi^{4}+8 A^{2} C^{2} v_{\mathrm{r} y}^{2} \xi^{4}+B^{2} C^{2} v_{\mathrm{r} y}^{2} \xi^{4}+23 D^{2} C^{2} v_{\mathrm{r} y}^{2} \xi^{4}+30 A^{2} D C v_{\mathrm{r} y}^{2} \xi^{4}+14 B^{2} D C v_{\mathrm{r} y}^{2} \xi^{4}+21 A^{4} v_{\mathrm{r} z}^{2} \xi^{4}+21 B^{4} v_{\mathrm{r} z}^{2} \xi^{4}$ $+42 A^{2} B^{2} v_{\mathrm{r} z}^{2} \xi^{4}+A^{2} D^{2} v_{\mathrm{r} z}^{2} \xi^{4}+B^{2} D^{2} v_{\mathrm{r} z}^{2} \xi^{4}+6 A^{2} C^{2} v_{\mathrm{r} z}^{2} \xi^{4}+6 B^{2} C^{2} v_{\mathrm{r} z}^{2} \xi^{4}+16 A^{2} D C v_{\mathrm{r} z}^{2} \xi^{4}+16 B^{2} D C v_{\mathrm{r} z}^{2} \xi^{4}+23 B^{4} v_{\mathrm{t} x}^{2} \xi^{4}$ $+23 A^{2} B^{2} v_{\mathrm{t} x}^{2} \xi^{4}+6 A^{2} D^{2} v_{\mathrm{t} x}^{2} \xi^{4}+8 B^{2} D^{2} v_{\mathrm{t} x}^{2} \xi^{4}+A^{2} C^{2} v_{\mathrm{t} x}^{2} \xi^{4}+8 B^{2} C^{2} v_{\mathrm{t} x}^{2} \xi^{4}+23 D^{2} C^{2} v_{\mathrm{t} x}^{2} \xi^{4}-14 A^{2} D C v_{\mathrm{t} x}^{2} \xi^{4}$ $-30 B^{2} D C v_{\mathrm{t} x}^{2} \xi^{4}+23 A^{4} v_{\mathrm{t} y}^{2} \xi^{4}+23 A^{2} B^{2} v_{\mathrm{t} y}^{2} \xi^{4}+8 A^{2} D^{2} v_{\mathrm{t} y}^{2} \xi^{4}+6 B^{2} D^{2} v_{\mathrm{t} y}^{2} \xi^{4}+8 A^{2} C^{2} v_{\mathrm{t} y}^{2} \xi^{4}+B^{2} C^{2} v_{\mathrm{t} y}^{2} \xi^{4}$ $+23 D^{2} C^{2} v_{\mathrm{t} y}^{2} \xi^{4}-30 A^{2} D C v_{\mathrm{t} y}^{2} \xi^{4}-14 B^{2} D C v_{\mathrm{t} y}^{2} \xi^{4}+21 A^{4} v_{\mathrm{t} z}^{2} \xi^{4}+21 B^{4} v_{\mathrm{t} z}^{2} \xi^{4}+42 A^{2} B^{2} v_{\mathrm{t} z}^{2} \xi^{4}+A^{2} D^{2} v_{\mathrm{t} z}^{2} \xi^{4}+B^{2} D^{2} v_{\mathrm{t} z}^{2} \xi^{4}$ $+6 A^{2} C^{2} v_{\mathrm{t} z}^{2} \xi^{4}+6 B^{2} C^{2} v_{\mathrm{t} z}^{2} \xi^{4}-16 A^{2} D C v_{\mathrm{t} z}^{2} \xi^{4}-16 B^{2} D C v_{\mathrm{t} z}^{2} \xi^{4}-46 A B^{3} v_{\mathrm{r} x} v_{\mathrm{r} y} \xi^{4}-4 A B D^{2} v_{\mathrm{r} x} v_{\mathrm{r} y} \xi^{4}-14 A B C^{2} v_{\mathrm{r} x} v_{\mathrm{r} y} \xi^{4}$ $-46 A^{3} B v_{\mathrm{r} x} v_{\mathrm{r} y} \xi^{4}-32 A B D C v_{\mathrm{r} x} v_{\mathrm{r} y} \xi^{4}-28 A D C^{2} v_{\mathrm{r} x} v_{\mathrm{r} z} \xi^{4}-26 A^{3} D v_{\mathrm{r} x} v_{\mathrm{r} z} \xi^{4}-26 A B^{2} D v_{\mathrm{r} x} v_{\mathrm{r} z} \xi^{4}-16 A^{3} C v_{\mathrm{r} x} v_{\mathrm{r} z} \xi^{4}$ $-16 A B^{2} C v_{\mathrm{r} x} v_{\mathrm{r} z} \xi^{4}-18 A D^{2} C v_{\mathrm{r} x} v_{\mathrm{r} z} \xi^{4}-28 B D C^{2} v_{\mathrm{r} y} v_{\mathrm{r} z} \xi^{4}-26 B^{3} D v_{\mathrm{r} y} v_{\mathrm{r} z} \xi^{4}-26 A^{2} B D v_{\mathrm{r} y} v_{\mathrm{r} z} \xi^{4}-16 B^{3} C v_{\mathrm{r} y} v_{\mathrm{r} z} \xi^{4}$ $-18 B D^{2} C v_{\mathrm{r} y} v_{\mathrm{r} z} \xi^{4}-16 A^{2} B C v_{\mathrm{r} y} v_{\mathrm{r} z} \xi^{4}-6 B^{4} v_{\mathrm{r} x} v_{\mathrm{t} x} \xi^{4}-6 A^{2} B^{2} v_{\mathrm{r} x} v_{\mathrm{t} x} \xi^{4}+4 A^{2} D^{2} v_{\mathrm{r} x} v_{\mathrm{t} x} \xi^{4}+2 A^{2} C^{2} v_{\mathrm{r} x} v_{\mathrm{t} x} \xi^{4}$ $+6 D^{2} C^{2} v_{\mathrm{r} x} v_{\mathrm{t} x} \xi^{4}+6 A B^{3} v_{\mathrm{r} y} v_{\mathrm{t} x} \xi^{4}+4 A B D^{2} v_{\mathrm{r} y} v_{\mathrm{t} x} \xi^{4}+2 A B C^{2} v_{\mathrm{r} y} v_{\mathrm{t} x} \xi^{4}+6 A^{3} B v_{\mathrm{r} y} v_{\mathrm{t} x} \xi^{4}-4 A D C^{2} v_{\mathrm{r} z} v_{\mathrm{t} x} \xi^{4}$ $-6 A^{3} D v_{\mathrm{r} z} v_{\mathrm{t} x} \xi^{4}-6 A B^{2} D v_{\mathrm{r} z} v_{\mathrm{t} x} \xi^{4}-2 A D^{2} C v_{\mathrm{r} z} v_{\mathrm{t} x} \xi^{4}+6 A B^{3} v_{\mathrm{r} x} v_{\mathrm{t} y} \xi^{4}+4 A B D^{2} v_{\mathrm{r} x} v_{\mathrm{t} y} \xi^{4}+2 A B C^{2} v_{\mathrm{r} x} v_{\mathrm{t} y} \xi^{4}$ $+6 A^{3} B v_{\mathrm{r} x} v_{\mathrm{t} y} \xi^{4}-6 A^{4} v_{\mathrm{r} y} v_{\mathrm{t} y} \xi^{4}-6 A^{2} B^{2} v_{\mathrm{r} y} v_{\mathrm{t} y} \xi^{4}+4 B^{2} D^{2} v_{\mathrm{r} y} v_{\mathrm{t} y} \xi^{4}+2 B^{2} C^{2} v_{\mathrm{r} y} v_{\mathrm{t} y} \xi^{4}+6 D^{2} C^{2} v_{\mathrm{r} y} v_{\mathrm{t} y} \xi^{4}$ 
$-4 B D C^{2} v_{\mathrm{r} z} v_{\mathrm{t} y} \xi^{4}-6 B^{3} D v_{\mathrm{r} z} v_{\mathrm{t} y} \xi^{4}-6 A^{2} B D v_{\mathrm{r} z} v_{\mathrm{t} y} \xi^{4}-2 B D^{2} C v_{\mathrm{r} z} v_{\mathrm{t} y} \xi^{4}-46 A B^{3} v_{\mathrm{t} x} v_{\mathrm{t} y} \xi^{4}-4 A B D^{2} v_{\mathrm{t} x} v_{\mathrm{t} y} \xi^{4}$

$-14 A B C^{2} v_{\mathrm{t} x} v_{\mathrm{t} y} \xi^{4}-46 A^{3} B v_{\mathrm{t} x} v_{\mathrm{t} y} \xi^{4}+32 A B D C v_{\mathrm{t} x} v_{\mathrm{t} y} \xi^{4}+4 A D C^{2} v_{\mathrm{r} x} v_{\mathrm{t} z} \xi^{4}+6 A^{3} D v_{\mathrm{r} x} v_{\mathrm{t} z} \xi^{4}+6 A B^{2} D v_{\mathrm{r} x} v_{\mathrm{t} z} \xi^{4}$

$-2 A D^{2} C v_{\mathrm{r} x} v_{\mathrm{t} z} \xi^{4}+4 B D C^{2} v_{\mathrm{r} y} v_{\mathrm{t} z} \xi^{4}+6 B^{3} D v_{\mathrm{r} y} v_{\mathrm{t} z} \xi^{4}+6 A^{2} B D v_{\mathrm{r} y} v_{\mathrm{t} z} \xi^{4}-2 B D^{2} C v_{\mathrm{r} y} v_{\mathrm{t} z} \xi^{4}-6 A^{4} v_{\mathrm{r} z} v_{\mathrm{t} z} \xi^{4}$

$-6 B^{4} v_{\mathrm{r} z} v_{\mathrm{t} z} \xi^{4}-12 A^{2} B^{2} v_{\mathrm{r} z} v_{\mathrm{t} z} \xi^{4}+2 A^{2} D^{2} v_{\mathrm{r} z} v_{\mathrm{t} z} \xi^{4}+2 B^{2} D^{2} v_{\mathrm{r} z} v_{\mathrm{t} z} \xi^{4}-4 A^{2} C^{2} v_{\mathrm{r} z} v_{\mathrm{t} z} \xi^{4}-4 B^{2} C^{2} v_{\mathrm{r} z} v_{\mathrm{t} z} \xi^{4}$

$+28 A D C^{2} v_{\mathrm{t} x} v_{\mathrm{t} z} \xi^{4}+26 A^{3} D v_{\mathrm{t} x} v_{\mathrm{t} z} \xi^{4}+26 A B^{2} D v_{\mathrm{t} x} v_{\mathrm{t} z} \xi^{4}-16 A^{3} C v_{\mathrm{t} x} v_{\mathrm{t} z} \xi^{4}-16 A B^{2} C v_{\mathrm{t} x} v_{\mathrm{t} z} \xi^{4}-18 A D^{2} C v_{\mathrm{t} x} v_{\mathrm{t} z} \xi^{4}$

$+28 B D C^{2} v_{\mathrm{t} y} v_{\mathrm{t} z} \xi^{4}+26 B^{3} D v_{\mathrm{t} y} v_{\mathrm{t} z} \xi^{4}+26 A^{2} B D v_{\mathrm{t} y} v_{\mathrm{t} z} \xi^{4}-16 B^{3} C v_{\mathrm{t} y} v_{\mathrm{t} z} \xi^{4}-18 B D^{2} C v_{\mathrm{t} y} v_{\mathrm{t} z} \xi^{4}-16 A^{2} B C v_{\mathrm{t} y} v_{\mathrm{t} z} \xi^{4}$

$-10 B^{4} v_{\mathrm{r} x}^{2} \xi^{2}-10 A^{2} B^{2} v_{\mathrm{r} x}^{2} \xi^{2}-7 A^{2} D^{2} v_{\mathrm{r} x}^{2} \xi^{2}-10 B^{2} D^{2} v_{\mathrm{r} x}^{2} \xi^{2}-10 D^{3} C v_{\mathrm{r} x}^{2} \xi^{2}-2 A^{2} D C v_{\mathrm{r} x}^{2} \xi^{2}-10 B^{2} D C v_{\mathrm{r} x}^{2} \xi^{2}$

$-10 A^{4} v_{\mathrm{r} y}^{2} \xi^{2}-10 A^{2} B^{2} v_{\mathrm{r} y}^{2} \xi^{2}-10 A^{2} D^{2} v_{\mathrm{r} y}^{2} \xi^{2}-7 B^{2} D^{2} v_{\mathrm{r} y}^{2} \xi^{2}-10 D^{3} C v_{\mathrm{r} y}^{2} \xi^{2}-10 A^{2} D C v_{\mathrm{r} y}^{2} \xi^{2}-2 B^{2} D C v_{\mathrm{r} y}^{2} \xi^{2}-9 A^{4} v_{\mathrm{r} z}^{2} \xi^{2}$

$-9 B^{4} v_{\mathrm{r} z}^{2} \xi^{2}-18 A^{2} B^{2} v_{\mathrm{r} z}^{2} \xi^{2}-2 A^{2} D^{2} v_{\mathrm{r} z}^{2} \xi^{2}-2 B^{2} D^{2} v_{\mathrm{r} z}^{2} \xi^{2}-8 A^{2} D C v_{\mathrm{r} z}^{2} \xi^{2}-8 B^{2} D C v_{\mathrm{r} z}^{2} \xi^{2}-10 B^{4} v_{\mathrm{t} x}^{2} \xi^{2}-10 A^{2} B^{2} v_{\mathrm{t} x}^{2} \xi^{2}$

$-7 A^{2} D^{2} v_{\mathrm{t} x}^{2} \xi^{2}-10 B^{2} D^{2} v_{\mathrm{t} x}^{2} \xi^{2}+10 D^{3} C v_{\mathrm{t} x}^{2} \xi^{2}+2 A^{2} D C v_{\mathrm{t} x}^{2} \xi^{2}+10 B^{2} D C v_{\mathrm{t} x}^{2} \xi^{2}-10 A^{4} v_{\mathrm{t} y}^{2} \xi^{2}-10 A^{2} B^{2} v_{\mathrm{t} y}^{2} \xi^{2}$

$-10 A^{2} D^{2} v_{\mathrm{t} y}^{2} \xi^{2}-7 B^{2} D^{2} v_{\mathrm{t} y}^{2} \xi^{2}+10 D^{3} C v_{\mathrm{t} y}^{2} \xi^{2}+10 A^{2} D C v_{\mathrm{t} y}^{2} \xi^{2}+2 B^{2} D C v_{\mathrm{t} y}^{2} \xi^{2}-9 A^{4} v_{\mathrm{t} z}^{2} \xi^{2}-9 B^{4} v_{\mathrm{t} z}^{2} \xi^{2}-18 A^{2} B^{2} v_{\mathrm{t} z}^{2} \xi^{2}$

$-2 A^{2} D^{2} v_{\mathrm{t} z}^{2} \xi^{2}-2 B^{2} D^{2} v_{\mathrm{t} z}^{2} \xi^{2}+8 A^{2} D C v_{\mathrm{t} z}^{2} \xi^{2}+8 B^{2} D C v_{\mathrm{t} z}^{2} \xi^{2}+20 A B^{3} v_{\mathrm{r} x} v_{\mathrm{r} y} \xi^{2}+6 A B D^{2} v_{\mathrm{r} x} v_{\mathrm{r} y} \xi^{2}+20 A^{3} B v_{\mathrm{r} x} v_{\mathrm{r} y} \xi^{2}$

$+16 A B D C v_{\mathrm{r} x} v_{\mathrm{r} y} \xi^{2}+2 A D^{3} v_{\mathrm{r} x} v_{\mathrm{r} z} \xi^{2}+16 A^{3} D v_{\mathrm{r} x} v_{\mathrm{r} z} \xi^{2}+16 A B^{2} D v_{\mathrm{r} x} v_{\mathrm{r} z} \xi^{2}+2 A^{3} C v_{\mathrm{r} x} v_{\mathrm{r} z} \xi^{2}+2 A B^{2} C v_{\mathrm{r} x} v_{\mathrm{r} z} \xi^{2}$

$+18 A D^{2} C v_{\mathrm{r} x} v_{\mathrm{r} z} \xi^{2}+2 B D^{3} v_{\mathrm{r} y} v_{\mathrm{r} z} \xi^{2}+16 B^{3} D v_{\mathrm{r} y} v_{\mathrm{r} z} \xi^{2}+16 A^{2} B D v_{\mathrm{r} y} v_{\mathrm{r} z} \xi^{2}+2 B^{3} C v_{\mathrm{r} y} v_{\mathrm{r} z} \xi^{2}+18 B D^{2} C v_{\mathrm{r} y} v_{\mathrm{r} z} \xi^{2}$

$+2 A^{2} B C v_{\mathrm{r} y} v_{\mathrm{r} z} \xi^{2}+4 B^{4} v_{\mathrm{r} x} v_{\mathrm{t} x} \xi^{2}+4 A^{2} B^{2} v_{\mathrm{r} x} v_{\mathrm{t} x} \xi^{2}-2 A^{2} D^{2} v_{\mathrm{r} x} v_{\mathrm{t} x} \xi^{2}+4 B^{2} D^{2} v_{\mathrm{r} x} v_{\mathrm{t} x} \xi^{2}-4 A B^{3} v_{\mathrm{r} y} v_{\mathrm{t} x} \xi^{2}$

$-6 A B D^{2} v_{\mathrm{r} y} v_{\mathrm{t} x} \xi^{2}-4 A^{3} B v_{\mathrm{r} y} v_{\mathrm{t} x} \xi^{2}-2 A D^{3} v_{\mathrm{r} z} v_{\mathrm{t} x} \xi^{2}+2 A^{3} C v_{\mathrm{r} z} v_{\mathrm{t} x} \xi^{2}+2 A B^{2} C v_{\mathrm{r} z} v_{\mathrm{t} x} \xi^{2}+2 A D^{2} C v_{\mathrm{r} z} v_{\mathrm{t} x} \xi^{2}$

$-4 A B^{3} v_{\mathrm{r} x} v_{\mathrm{t} y} \xi^{2}-6 A B D^{2} v_{\mathrm{r} x} v_{\mathrm{t} y} \xi^{2}-4 A^{3} B v_{\mathrm{r} x} v_{\mathrm{t} y} \xi^{2}+4 A^{4} v_{\mathrm{r} y} v_{\mathrm{t} y} \xi^{2}+4 A^{2} B^{2} v_{\mathrm{r} y} v_{\mathrm{t} y} \xi^{2}+4 A^{2} D^{2} v_{\mathrm{r} y} v_{\mathrm{t} y} \xi^{2}$

$-2 B^{2} D^{2} v_{\mathrm{r} y} v_{\mathrm{t} y} \xi^{2}-2 B D^{3} v_{\mathrm{r} z} v_{\mathrm{t} y} \xi^{2}+2 B^{3} C v_{\mathrm{r} z} v_{\mathrm{t} y} \xi^{2}+2 B D^{2} C v_{\mathrm{r} z} v_{\mathrm{t} y} \xi^{2}+2 A^{2} B C v_{\mathrm{r} z} v_{\mathrm{t} y} \xi^{2}+20 A B^{3} v_{\mathrm{t} x} v_{\mathrm{t} y} \xi^{2}$

$+6 A B D^{2} v_{\mathrm{t} x} v_{\mathrm{t} y} \xi^{2}+20 A^{3} B v_{\mathrm{t} x} v_{\mathrm{t} y} \xi^{2}-16 A B D C v_{\mathrm{t} x} v_{\mathrm{t} y} \xi^{2}+2 A D^{3} v_{\mathrm{r} x} v_{\mathrm{t} z} \xi^{2}+2 A^{3} C v_{\mathrm{r} x} v_{\mathrm{t} z} \xi^{2}+2 A B^{2} C v_{\mathrm{r} x} v_{\mathrm{t} z} \xi^{2}$

$+2 A D^{2} C v_{\mathrm{r} x} v_{\mathrm{t} z} \xi^{2}+2 B D^{3} v_{\mathrm{r} y} v_{\mathrm{t} z} \xi^{2}+2 B^{3} C v_{\mathrm{r} y} v_{\mathrm{t} z} \xi^{2}+2 B D^{2} C v_{\mathrm{r} y} v_{\mathrm{t} z} \xi^{2}+2 A^{2} B C v_{\mathrm{r} y} v_{\mathrm{t} z} \xi^{2}-2 A^{4} v_{\mathrm{r} z} v_{\mathrm{t} z} \xi^{2}-2 B^{4} v_{\mathrm{r} z} v_{\mathrm{t} z} \xi^{2}$

$-4 A^{2} B^{2} v_{\mathrm{r} z} v_{\mathrm{t} z} \xi^{2}-4 A^{2} D^{2} v_{\mathrm{r} z} v_{\mathrm{t} z} \xi^{2}-4 B^{2} D^{2} v_{\mathrm{r} z} v_{\mathrm{t} z} \xi^{2}-2 A D^{3} v_{\mathrm{t} x} v_{\mathrm{t} z} \xi^{2}-16 A^{3} D v_{\mathrm{t} x} v_{\mathrm{t} z} \xi^{2}-16 A B^{2} D v_{\mathrm{t} x} v_{\mathrm{t} z} \xi^{2}$

$+2 A^{3} C v_{\mathrm{t} x} v_{\mathrm{t} z} \xi^{2}+2 A B^{2} C v_{\mathrm{t} x} v_{\mathrm{t} z} \xi^{2}+18 A D^{2} C v_{\mathrm{t} x} v_{\mathrm{t} z} \xi^{2}-2 B D^{3} v_{\mathrm{t} y} v_{\mathrm{t} z} \xi^{2}-16 B^{3} D v_{\mathrm{t} y} v_{\mathrm{t} z} \xi^{2}-16 A^{2} B D v_{\mathrm{t} y} v_{\mathrm{t} z} \xi^{2}$

$+2 B^{3} C v_{\mathrm{t} y} v_{\mathrm{t} z} \xi^{2}+18 B D^{2} C v_{\mathrm{t} y} v_{\mathrm{t} z} \xi^{2}+2 A^{2} B C v_{\mathrm{t} y} v_{\mathrm{t} z} \xi^{2}+B^{4} v_{\mathrm{r} x}^{2}+D^{4} v_{\mathrm{r} x}^{2}+A^{2} B^{2} v_{\mathrm{r} x}^{2}+A^{2} D^{2} v_{\mathrm{r} x}^{2}+2 B^{2} D^{2} v_{\mathrm{r} x}^{2}+A^{4} v_{\mathrm{r} y}^{2}$

$+D^{4} v_{\mathrm{r} y}^{2}+A^{2} B^{2} v_{\mathrm{r} y}^{2}+2 A^{2} D^{2} v_{\mathrm{r} y}^{2}+B^{2} D^{2} v_{\mathrm{r} y}^{2}+A^{4} v_{\mathrm{r} z}^{2}+B^{4} v_{\mathrm{r} z}^{2}+2 A^{2} B^{2} v_{\mathrm{r} z}^{2}+A^{2} D^{2} v_{\mathrm{r} z}^{2}+B^{2} D^{2} v_{\mathrm{r} z}^{2}+B^{4} v_{\mathrm{t} x}^{2}+D^{4} v_{\mathrm{t} x}^{2}$

$+A^{2} B^{2} v_{\mathrm{t} x}^{2}+A^{2} D^{2} v_{\mathrm{t} x}^{2}+2 B^{2} D^{2} v_{\mathrm{t} x}^{2}+A^{4} v_{\mathrm{t} y}^{2}+D^{4} v_{\mathrm{t} y}^{2}+A^{2} B^{2} v_{\mathrm{t} y}^{2}+2 A^{2} D^{2} v_{\mathrm{t} y}^{2}+B^{2} D^{2} v_{\mathrm{t} y}^{2}+A^{4} v_{\mathrm{t} z}^{2}+B^{4} v_{\mathrm{t} z}^{2}+2 A^{2} B^{2} v_{\mathrm{t} z}^{2}$

$+A^{2} D^{2} v_{\mathrm{t} z}^{2}+B^{2} D^{2} v_{\mathrm{t} z}^{2}-2 A B^{3} v_{\mathrm{r} x} v_{\mathrm{r} y}-2 A B D^{2} v_{\mathrm{r} x} v_{\mathrm{r} y}-2 A^{3} B v_{\mathrm{r} x} v_{\mathrm{r} y}-2 A D^{3} v_{\mathrm{r} x} v_{\mathrm{r} z}-2 A^{3} D v_{\mathrm{r} x} v_{\mathrm{r} z}-2 A B^{2} D v_{\mathrm{r} x} v_{\mathrm{r} z}$

$-2 B D^{3} v_{\mathrm{r} y} v_{\mathrm{r} z}-2 B^{3} D v_{\mathrm{r} y} v_{\mathrm{r} z}-2 A^{2} B D v_{\mathrm{r} y} v_{\mathrm{r} z}-2 B^{4} v_{\mathrm{r} x} v_{\mathrm{t} x}-2 D^{4} v_{\mathrm{r} x} v_{\mathrm{t} x}-2 A^{2} B^{2} v_{\mathrm{r} x} v_{\mathrm{t} x}-2 A^{2} D^{2} v_{\mathrm{r} x} v_{\mathrm{t} x}-4 B^{2} D^{2} v_{\mathrm{r} x} v_{\mathrm{t} x}$

$+2 A B^{3} v_{\mathrm{r} y} v_{\mathrm{t} x}+2 A B D^{2} v_{\mathrm{r} y} v_{\mathrm{t} x}+2 A^{3} B v_{\mathrm{r} y} v_{\mathrm{t} x}+2 A D^{3} v_{\mathrm{r} z} v_{\mathrm{t} x}+2 A^{3} D v_{\mathrm{r} z} v_{\mathrm{t} x}+2 A B^{2} D v_{\mathrm{r} z} v_{\mathrm{t} x}+2 A B^{3} v_{\mathrm{r} x} v_{\mathrm{t} y}+2 A B D^{2} v_{\mathrm{r} x} v_{\mathrm{t} y}$

$+2 A^{3} B v_{\mathrm{r} x} v_{\mathrm{t} y}-2 A^{4} v_{\mathrm{r} y} v_{\mathrm{t} y}-2 D^{4} v_{\mathrm{r} y} v_{\mathrm{t} y}-2 A^{2} B^{2} v_{\mathrm{r} y} v_{\mathrm{t} y}-4 A^{2} D^{2} v_{\mathrm{r} y} v_{\mathrm{t} y}-2 B^{2} D^{2} v_{\mathrm{r} y} v_{\mathrm{t} y}+2 B D^{3} v_{\mathrm{r} z} v_{\mathrm{t} y}+2 B^{3} D v_{\mathrm{r} z} v_{\mathrm{t} y}$

$+2 A^{2} B D v_{\mathrm{r} z} v_{\mathrm{t} y}-2 A B^{3} v_{\mathrm{t} x} v_{\mathrm{t} y}-2 A B D^{2} v_{\mathrm{t} x} v_{\mathrm{t} y}-2 A^{3} B v_{\mathrm{t} x} v_{\mathrm{t} y}-2 A D^{3} v_{\mathrm{r} x} v_{\mathrm{t} z}-2 A^{3} D v_{\mathrm{r} x} v_{\mathrm{t} z}-2 A B^{2} D v_{\mathrm{r} x} v_{\mathrm{t} z}-2 B D^{3} v_{\mathrm{r} y} v_{\mathrm{t} z}$

$-2 B^{3} D v_{\mathrm{r} y} v_{\mathrm{t} z}-2 A^{2} B D v_{\mathrm{r} y} v_{\mathrm{t} z}+2 A^{4} v_{\mathrm{r} z} v_{\mathrm{t} z}+2 B^{4} v_{\mathrm{r} z} v_{\mathrm{t} z}+4 A^{2} B^{2} v_{\mathrm{r} z} v_{\mathrm{t} z}+2 A^{2} D^{2} v_{\mathrm{r} z} v_{\mathrm{t} z}+2 B^{2} D^{2} v_{\mathrm{r} z} v_{\mathrm{t} z}+2 A D^{3} v_{\mathrm{t} x} v_{\mathrm{t} z}$

$\left.+2 A^{3} D v_{\mathrm{t} x} v_{\mathrm{t} z}+2 A B^{2} D v_{\mathrm{t} x} v_{\mathrm{t} z}+2 B D^{3} v_{\mathrm{t} y} v_{\mathrm{t} z}+2 B^{3} D v_{\mathrm{t} y} v_{\mathrm{t} z}+2 A^{2} B D v_{\mathrm{t} y} v_{\mathrm{t} z}\right) \eta^{4}$ 
$+\left(4 B^{4} v_{\mathrm{r} x}^{2} \xi^{9}+4 C^{4} v_{\mathrm{r} x}^{2} \xi^{9}+4 A^{2} B^{2} v_{\mathrm{r} x}^{2} \xi^{9}+4 A^{2} C^{2} v_{\mathrm{r} x}^{2} \xi^{9}+8 B^{2} C^{2} v_{\mathrm{r} x}^{2} \xi^{9}+4 A^{4} v_{\mathrm{r} y}^{2} \xi^{9}+4 C^{4} v_{\mathrm{r} y}^{2} \xi^{9}+4 A^{2} B^{2} v_{\mathrm{r} y}^{2} \xi^{9}\right.$ $+8 A^{2} C^{2} v_{\mathrm{r} y}^{2} \xi^{9}+4 B^{2} C^{2} v_{\mathrm{r} y}^{2} \xi^{9}+4 A^{4} v_{\mathrm{r} z}^{2} \xi^{9}+4 B^{4} v_{\mathrm{r} z}^{2} \xi^{9}+8 A^{2} B^{2} v_{\mathrm{r} z}^{2} \xi^{9}+4 A^{2} C^{2} v_{\mathrm{r} z}^{2} \xi^{9}+4 B^{2} C^{2} v_{\mathrm{r} z}^{2} \xi^{9}-4 B^{4} v_{\mathrm{t} x}^{2} \xi^{9}$ $-4 C^{4} v_{\mathrm{t} x}^{2} \xi^{9}-4 A^{2} B^{2} v_{\mathrm{t} x}^{2} \xi^{9}-4 A^{2} C^{2} v_{\mathrm{t} x}^{2} \xi^{9}-8 B^{2} C^{2} v_{\mathrm{t} x}^{2} \xi^{9}-4 A^{4} v_{\mathrm{t} y}^{2} \xi^{9}-4 C^{4} v_{\mathrm{t} y}^{2} \xi^{9}-4 A^{2} B^{2} v_{\mathrm{t} y}^{2} \xi^{9}-8 A^{2} C^{2} v_{\mathrm{t} y}^{2} \xi^{9}$ $-4 B^{2} C^{2} v_{\mathrm{t} y}^{2} \xi^{9}-4 A^{4} v_{\mathrm{t} z}^{2} \xi^{9}-4 B^{4} v_{\mathrm{t} z}^{2} \xi^{9}-8 A^{2} B^{2} v_{\mathrm{t} z}^{2} \xi^{9}-4 A^{2} C^{2} v_{\mathrm{t} z}^{2} \xi^{9}-4 B^{2} C^{2} v_{\mathrm{t} z}^{2} \xi^{9}-8 A B^{3} v_{\mathrm{r} x} v_{\mathrm{r} y} \xi^{9}-8 A B C^{2} v_{\mathrm{r} x} v_{\mathrm{r} y} \xi^{9}$ $-8 A^{3} B v_{\mathrm{r} x} v_{\mathrm{r} y} \xi^{9}-8 A C^{3} v_{\mathrm{r} x} v_{\mathrm{r} z} \xi^{9}-8 A^{3} C v_{\mathrm{r} x} v_{\mathrm{r} z} \xi^{9}-8 A B^{2} C v_{\mathrm{r} x} v_{\mathrm{r} z} \xi^{9}-8 B C^{3} v_{\mathrm{r} y} v_{\mathrm{r} z} \xi^{9}-8 B^{3} C v_{\mathrm{r} y} v_{\mathrm{r} z} \xi^{9}$ $-8 A^{2} B C v_{\mathrm{r} y} v_{\mathrm{r} z} \xi^{9}+8 A B^{3} v_{\mathrm{t} x} v_{\mathrm{t} y} \xi^{9}+8 A B C^{2} v_{\mathrm{t} x} v_{\mathrm{t} y} \xi^{9}+8 A^{3} B v_{\mathrm{t} x} v_{\mathrm{t} y} \xi^{9}+8 A C^{3} v_{\mathrm{t} x} v_{\mathrm{t} z} \xi^{9}+8 A^{3} C v_{\mathrm{t} x} v_{\mathrm{t} z} \xi^{9}$ $+8 A B^{2} C v_{\mathrm{t} x} v_{\mathrm{t} z} \xi^{9}+8 B C^{3} v_{\mathrm{t} y} v_{\mathrm{t} z} \xi^{9}+8 B^{3} C v_{\mathrm{t} y} v_{\mathrm{t} z} \xi^{9}+8 A^{2} B C v_{\mathrm{t} y} v_{\mathrm{t} z} \xi^{9}-20 B^{4} v_{\mathrm{r} x}^{2} \xi^{7}-20 D C^{3} v_{\mathrm{r} x}^{2} \xi^{7}-20 A^{2} B^{2} v_{\mathrm{r} x}^{2} \xi^{7}$ $-8 A^{2} C^{2} v_{\mathrm{r} x}^{2} \xi^{7}-20 B^{2} C^{2} v_{\mathrm{r} x}^{2} \xi^{7}-8 A^{2} D C v_{\mathrm{r} x}^{2} \xi^{7}-20 B^{2} D C v_{\mathrm{r} x}^{2} \xi^{7}-20 A^{4} v_{\mathrm{r} y}^{2} \xi^{7}-20 D C^{3} v_{\mathrm{r} y}^{2} \xi^{7}-20 A^{2} B^{2} v_{\mathrm{r} y}^{2} \xi^{7}$ $-20 A^{2} C^{2} v_{\mathrm{r} y}^{2} \xi^{7}-8 B^{2} C^{2} v_{\mathrm{r} y}^{2} \xi^{7}-20 A^{2} D C v_{\mathrm{r} y}^{2} \xi^{7}-8 B^{2} D C v_{\mathrm{r} y}^{2} \xi^{7}-16 A^{4} v_{\mathrm{r} z}^{2} \xi^{7}-16 B^{4} v_{\mathrm{r} z}^{2} \xi^{7}-32 A^{2} B^{2} v_{\mathrm{r} z}^{2} \xi^{7}$ $-8 A^{2} C^{2} v_{\mathrm{r} z}^{2} \xi^{7}-8 B^{2} C^{2} v_{\mathrm{r} z}^{2} \xi^{7}-12 A^{2} D C v_{\mathrm{r} z}^{2} \xi^{7}-12 B^{2} D C v_{\mathrm{r} z}^{2} \xi^{7}+20 B^{4} v_{\mathrm{t} x}^{2} \xi^{7}-20 D C^{3} v_{\mathrm{t} x}^{2} \xi^{7}+20 A^{2} B^{2} v_{\mathrm{t} x}^{2} \xi^{7}$ $+8 A^{2} C^{2} v_{\mathrm{t} x}^{2} \xi^{7}+20 B^{2} C^{2} v_{\mathrm{t} x}^{2} \xi^{7}-8 A^{2} D C v_{\mathrm{t} x}^{2} \xi^{7}-20 B^{2} D C v_{\mathrm{t} x}^{2} \xi^{7}+20 A^{4} v_{\mathrm{t} y}^{2} \xi^{7}-20 D C^{3} v_{\mathrm{t} y}^{2} \xi^{7}+20 A^{2} B^{2} v_{\mathrm{t} y}^{2} \xi^{7}$ $+20 A^{2} C^{2} v_{\mathrm{t} y}^{2} \xi^{7}+8 B^{2} C^{2} v_{\mathrm{t} y}^{2} \xi^{7}-20 A^{2} D C v_{\mathrm{t} y}^{2} \xi^{7}-8 B^{2} D C v_{\mathrm{t} y}^{2} \xi^{7}+16 A^{4} v_{\mathrm{t} z}^{2} \xi^{7}+16 B^{4} v_{\mathrm{t} z}^{2} \xi^{7}+32 A^{2} B^{2} v_{\mathrm{t} z}^{2} \xi^{7}$ $+8 A^{2} C^{2} v_{\mathrm{t} z}^{2} \xi^{7}+8 B^{2} C^{2} v_{\mathrm{t} z}^{2} \xi^{7}-12 A^{2} D C v_{\mathrm{t} z}^{2} \xi^{7}-12 B^{2} D C v_{\mathrm{t} z}^{2} \xi^{7}+40 A B^{3} v_{\mathrm{r} x} v_{\mathrm{r} y} \xi^{7}+24 A B C^{2} v_{\mathrm{r} x} v_{\mathrm{r} y} \xi^{7}+40 A^{3} B v_{\mathrm{r} x} v_{\mathrm{r} y} \xi^{7}$ $+24 A B D C v_{\mathrm{r} x} v_{\mathrm{r} y} \xi^{7}+8 A C^{3} v_{\mathrm{r} x} v_{\mathrm{r} z} \xi^{7}+32 A D C^{2} v_{\mathrm{r} x} v_{\mathrm{r} z} \xi^{7}+8 A^{3} D v_{\mathrm{r} x} v_{\mathrm{r} z} \xi^{7}+8 A B^{2} D v_{\mathrm{r} x} v_{\mathrm{r} z} \xi^{7}+24 A^{3} C v_{\mathrm{r} x} v_{\mathrm{r} z} \xi^{7}$ $+24 A B^{2} C v_{\mathrm{r} x} v_{\mathrm{r} z} \xi^{7}+8 B C^{3} v_{\mathrm{r} y} v_{\mathrm{r} z} \xi^{7}+32 B D C^{2} v_{\mathrm{r} y} v_{\mathrm{r} z} \xi^{7}+8 B^{3} D v_{\mathrm{r} y} v_{\mathrm{r} z} \xi^{7}+8 A^{2} B D v_{\mathrm{r} y} v_{\mathrm{r} z} \xi^{7}+24 B^{3} C v_{\mathrm{r} y} v_{\mathrm{r} z} \xi^{7}$ $+24 A^{2} B C v_{\mathrm{r} y} v_{\mathrm{r} z} \xi^{7}+8 D C^{3} v_{\mathrm{r} x} v_{\mathrm{t} x} \xi^{7}+8 B^{2} D C v_{\mathrm{r} x} v_{\mathrm{t} x} \xi^{7}-8 A B D C v_{\mathrm{r} y} v_{\mathrm{t} x} \xi^{7}-8 A D C^{2} v_{\mathrm{r} z} v_{\mathrm{t} x} \xi^{7}-8 A B D C v_{\mathrm{r} x} v_{\mathrm{t} y} \xi^{7}$ $+8 D C^{3} v_{\mathrm{r} y} v_{\mathrm{t} y} \xi^{7}+8 A^{2} D C v_{\mathrm{r} y} v_{\mathrm{t} y} \xi^{7}-8 B D C^{2} v_{\mathrm{r} z} v_{\mathrm{t} y} \xi^{7}-40 A B^{3} v_{\mathrm{t} x} v_{\mathrm{t} y} \xi^{7}-24 A B C^{2} v_{\mathrm{t} x} v_{\mathrm{t} y} \xi^{7}-40 A^{3} B v_{\mathrm{t} x} v_{\mathrm{t} y} \xi^{7}$ $+24 A B D C v_{\mathrm{t} x} v_{\mathrm{t} y} \xi^{7}-8 A D C^{2} v_{\mathrm{r} x} v_{\mathrm{t} z} \xi^{7}-8 B D C^{2} v_{\mathrm{r} y} v_{\mathrm{t} z} \xi^{7}+8 A^{2} D C v_{\mathrm{r} z} v_{\mathrm{t} z} \xi^{7}+8 B^{2} D C v_{\mathrm{r} z} v_{\mathrm{t} z} \xi^{7}-8 A C^{3} v_{\mathrm{t} x} v_{\mathrm{t} z} \xi^{7}$ $+32 A D C^{2} v_{\mathrm{t} x} v_{\mathrm{t} z} \xi^{7}+8 A^{3} D v_{\mathrm{t} x} v_{\mathrm{t} z} \xi^{7}+8 A B^{2} D v_{\mathrm{t} x} v_{\mathrm{t} z} \xi^{7}-24 A^{3} C v_{\mathrm{t} x} v_{\mathrm{t} z} \xi^{7}-24 A B^{2} C v_{\mathrm{t} x} v_{\mathrm{t} z} \xi^{7}-8 B C^{3} v_{\mathrm{t} y} v_{\mathrm{t} z} \xi^{7}$ $+32 B D C^{2} v_{\mathrm{t} y} v_{\mathrm{t} z} \xi^{7}+8 B^{3} D v_{\mathrm{t} y} v_{\mathrm{t} z} \xi^{7}+8 A^{2} B D v_{\mathrm{t} y} v_{\mathrm{t} z} \xi^{7}-24 B^{3} C v_{\mathrm{t} y} v_{\mathrm{t} z} \xi^{7}-24 A^{2} B C v_{\mathrm{t} y} v_{\mathrm{t} z} \xi^{7}+32 B^{4} v_{\mathrm{r} x}^{2} \xi^{5}$ $+32 A^{2} B^{2} v_{\mathrm{r} x}^{2} \xi^{5}+4 A^{2} D^{2} v_{\mathrm{r} x}^{2} \xi^{5}+12 B^{2} D^{2} v_{\mathrm{r} x}^{2} \xi^{5}+4 A^{2} C^{2} v_{\mathrm{r} x}^{2} \xi^{5}+12 B^{2} C^{2} v_{\mathrm{r} x}^{2} \xi^{5}+32 D^{2} C^{2} v_{\mathrm{r} x}^{2} \xi^{5}+16 A^{2} D C v_{\mathrm{r} x}^{2} \xi^{5}$ $+40 B^{2} D C v_{\mathrm{r} x}^{2} \xi^{5}+32 A^{4} v_{\mathrm{r} y}^{2} \xi^{5}+32 A^{2} B^{2} v_{\mathrm{r} y}^{2} \xi^{5}+12 A^{2} D^{2} v_{\mathrm{r} y}^{2} \xi^{5}+4 B^{2} D^{2} v_{\mathrm{r} y}^{2} \xi^{5}+12 A^{2} C^{2} v_{\mathrm{r} y}^{2} \xi^{5}+4 B^{2} C^{2} v_{\mathrm{r} y}^{2} \xi^{5}$ $+32 D^{2} C^{2} v_{\mathrm{r} y}^{2} \xi^{5}+40 A^{2} D C v_{\mathrm{r} y}^{2} \xi^{5}+16 B^{2} D C v_{\mathrm{r} y}^{2} \xi^{5}+24 A^{4} v_{\mathrm{r} z}^{2} \xi^{5}+24 B^{4} v_{\mathrm{r} z}^{2} \xi^{5}+48 A^{2} B^{2} v_{\mathrm{r} z}^{2} \xi^{5}+4 A^{2} D^{2} v_{\mathrm{r} z}^{2} \xi^{5}$ $+4 B^{2} D^{2} v_{\mathrm{r} z}^{2} \xi^{5}+4 A^{2} C^{2} v_{\mathrm{r} z}^{2} \xi^{5}+4 B^{2} C^{2} v_{\mathrm{r} z}^{2} \xi^{5}+24 A^{2} D C v_{\mathrm{r} z}^{2} \xi^{5}+24 B^{2} D C v_{\mathrm{r} z}^{2} \xi^{5}-32 B^{4} v_{\mathrm{t} x}^{2} \xi^{5}-32 A^{2} B^{2} v_{\mathrm{t} x}^{2} \xi^{5}$ $-4 A^{2} D^{2} v_{\mathrm{t} x}^{2} \xi^{5}-12 B^{2} D^{2} v_{\mathrm{t} x}^{2} \xi^{5}-4 A^{2} C^{2} v_{\mathrm{t} x}^{2} \xi^{5}-12 B^{2} C^{2} v_{\mathrm{t} x}^{2} \xi^{5}-32 D^{2} C^{2} v_{\mathrm{t} x}^{2} \xi^{5}+16 A^{2} D C v_{\mathrm{t} x}^{2} \xi^{5}+40 B^{2} D C v_{\mathrm{t} x}^{2} \xi^{5}$ $-32 A^{4} v_{\mathrm{t} y}^{2} \xi^{5}-32 A^{2} B^{2} v_{\mathrm{t} y}^{2} \xi^{5}-12 A^{2} D^{2} v_{\mathrm{t} y}^{2} \xi^{5}-4 B^{2} D^{2} v_{\mathrm{t} y}^{2} \xi^{5}-12 A^{2} C^{2} v_{\mathrm{t} y}^{2} \xi^{5}-4 B^{2} C^{2} v_{\mathrm{t} y}^{2} \xi^{5}-32 D^{2} C^{2} v_{\mathrm{t} y}^{2} \xi^{5}$ $+40 A^{2} D C v_{\mathrm{t} y}^{2} \xi^{5}+16 B^{2} D C v_{\mathrm{t} y}^{2} \xi^{5}-24 A^{4} v_{\mathrm{t} z}^{2} \xi^{5}-24 B^{4} v_{\mathrm{t} z}^{2} \xi^{5}-48 A^{2} B^{2} v_{\mathrm{t} z}^{2} \xi^{5}-4 A^{2} D^{2} v_{\mathrm{t} z}^{2} \xi^{5}-4 B^{2} D^{2} v_{\mathrm{t} z}^{2} \xi^{5}-4 A^{2} C^{2} v_{\mathrm{t} z}^{2} \xi^{5}$ $-4 B^{2} C^{2} v_{\mathrm{t} z}^{2} \xi^{5}+24 A^{2} D C v_{\mathrm{t} z}^{2} \xi^{5}+24 B^{2} D C v_{\mathrm{t} z}^{2} \xi^{5}-64 A B^{3} v_{\mathrm{r} x} v_{\mathrm{r} y} \xi^{5}-16 A B D^{2} v_{\mathrm{r} x} v_{\mathrm{r} y} \xi^{5}-16 A B C^{2} v_{\mathrm{r} x} v_{\mathrm{r} y} \xi^{5}$ $-64 A^{3} B v_{\mathrm{r} x} v_{\mathrm{r} y} \xi^{5}-48 A B D C v_{\mathrm{r} x} v_{\mathrm{r} y} \xi^{5}-32 A D C^{2} v_{\mathrm{r} x} v_{\mathrm{r} z} \xi^{5}-24 A^{3} D v_{\mathrm{r} x} v_{\mathrm{r} z} \xi^{5}-24 A B^{2} D v_{\mathrm{r} x} v_{\mathrm{r} z} \xi^{5}-24 A^{3} C v_{\mathrm{r} x} v_{\mathrm{r} z} \xi^{5}$ $-24 A B^{2} C v_{\mathrm{r} x} v_{\mathrm{r} z} \xi^{5}-32 A D^{2} C v_{\mathrm{r} x} v_{\mathrm{r} z} \xi^{5}-32 B D C^{2} v_{\mathrm{r} y} v_{\mathrm{r} z} \xi^{5}-24 B^{3} D v_{\mathrm{r} y} v_{\mathrm{r} z} \xi^{5}-24 A^{2} B D v_{\mathrm{r} y} v_{\mathrm{r} z} \xi^{5}-24 B^{3} C v_{\mathrm{r} y} v_{\mathrm{r} z} \xi^{5}$ $-32 B D^{2} C v_{\mathrm{r} y} v_{\mathrm{r} z} \xi^{5}-24 A^{2} B C v_{\mathrm{r} y} v_{\mathrm{r} z} \xi^{5}+8 A D C^{2} v_{\mathrm{r} z} v_{\mathrm{t} x} \xi^{5}-8 A D^{2} C v_{\mathrm{r} z} v_{\mathrm{t} x} \xi^{5}+8 B D C^{2} v_{\mathrm{r} z} v_{\mathrm{t} y} \xi^{5}-8 B D^{2} C v_{\mathrm{r} z} v_{\mathrm{t} y} \xi^{5}$ $+64 A B^{3} v_{\mathrm{t} x} v_{\mathrm{t} y} \xi^{5}+16 A B D^{2} v_{\mathrm{t} x} v_{\mathrm{t} y} \xi^{5}+16 A B C^{2} v_{\mathrm{t} x} v_{\mathrm{t} y} \xi^{5}+64 A^{3} B v_{\mathrm{t} x} v_{\mathrm{t} y} \xi^{5}-48 A B D C v_{\mathrm{t} x} v_{\mathrm{t} y} \xi^{5}+8 A D C^{2} v_{\mathrm{r} x} v_{\mathrm{t} z} \xi^{5}$ $+8 A D^{2} C v_{\mathrm{r} x} v_{\mathrm{t} z} \xi^{5}+8 B D C^{2} v_{\mathrm{r} y} v_{\mathrm{t} z} \xi^{5}+8 B D^{2} C v_{\mathrm{r} y} v_{\mathrm{t} z} \xi^{5}-16 A^{2} D C v_{\mathrm{r} z} v_{\mathrm{t} z} \xi^{5}-16 B^{2} D C v_{\mathrm{r} z} v_{\mathrm{t} z} \xi^{5}-32 A D C^{2} v_{\mathrm{t} x} v_{\mathrm{t} z} \xi^{5}$ $-24 A^{3} D v_{\mathrm{t} x} v_{\mathrm{t} z} \xi^{5}-24 A B^{2} D v_{\mathrm{t} x} v_{\mathrm{t} z} \xi^{5}+24 A^{3} C v_{\mathrm{t} x} v_{\mathrm{t} z} \xi^{5}+24 A B^{2} C v_{\mathrm{t} x} v_{\mathrm{t} z} \xi^{5}+32 A D^{2} C v_{\mathrm{t} x} v_{\mathrm{t} z} \xi^{5}-32 B D C^{2} v_{\mathrm{t} y} v_{\mathrm{t} z} \xi^{5}$ $-24 B^{3} D v_{\mathrm{t} y} v_{\mathrm{t} z} \xi^{5}-24 A^{2} B D v_{\mathrm{t} y} v_{\mathrm{t} z} \xi^{5}+24 B^{3} C v_{\mathrm{t} y} v_{\mathrm{t} z} \xi^{5}+32 B D^{2} C v_{\mathrm{t} y} v_{\mathrm{t} z} \xi^{5}+24 A^{2} B C v_{\mathrm{t} y} v_{\mathrm{t} z} \xi^{5}-20 B^{4} v_{\mathrm{r} x}^{2} \xi^{3}$ $-20 A^{2} B^{2} v_{\mathrm{r} x}^{2} \xi^{3}-8 A^{2} D^{2} v_{\mathrm{r} x}^{2} \xi^{3}-20 B^{2} D^{2} v_{\mathrm{r} x}^{2} \xi^{3}-20 D^{3} C v_{\mathrm{r} x}^{2} \xi^{3}-8 A^{2} D C v_{\mathrm{r} x}^{2} \xi^{3}-20 B^{2} D C v_{\mathrm{r} x}^{2} \xi^{3}-20 A^{4} v_{\mathrm{r} y}^{2} \xi^{3}$ $-20 A^{2} B^{2} v_{\mathrm{r} y}^{2} \xi^{3}-20 A^{2} D^{2} v_{\mathrm{r} y}^{2} \xi^{3}-8 B^{2} D^{2} v_{\mathrm{r} y}^{2} \xi^{3}-20 D^{3} C v_{\mathrm{r} y}^{2} \xi^{3}-20 A^{2} D C v_{\mathrm{r} y}^{2} \xi^{3}-8 B^{2} D C v_{\mathrm{r} y}^{2} \xi^{3}-16 A^{4} v_{\mathrm{r} z}^{2} \xi^{3}$ $-16 B^{4} v_{\mathrm{r} z}^{2} \xi^{3}-32 A^{2} B^{2} v_{\mathrm{r} z}^{2} \xi^{3}-8 A^{2} D^{2} v_{\mathrm{r} z}^{2} \xi^{3}-8 B^{2} D^{2} v_{\mathrm{r} z}^{2} \xi^{3}-12 A^{2} D C v_{\mathrm{r} z}^{2} \xi^{3}-12 B^{2} D C v_{\mathrm{r} z}^{2} \xi^{3}+20 B^{4} v_{\mathrm{t} x}^{2} \xi^{3}$ $+20 A^{2} B^{2} v_{\mathrm{t} x}^{2} \xi^{3}+8 A^{2} D^{2} v_{\mathrm{t} x}^{2} \xi^{3}+20 B^{2} D^{2} v_{\mathrm{t} x}^{2} \xi^{3}-20 D^{3} C v_{\mathrm{t} x}^{2} \xi^{3}-8 A^{2} D C v_{\mathrm{t} x}^{2} \xi^{3}-20 B^{2} D C v_{\mathrm{t} x}^{2} \xi^{3}+20 A^{4} v_{\mathrm{t} y}^{2} \xi^{3}$ $+20 A^{2} B^{2} v_{\mathrm{t} y}^{2} \xi^{3}+20 A^{2} D^{2} v_{\mathrm{t} y}^{2} \xi^{3}+8 B^{2} D^{2} v_{\mathrm{t} y}^{2} \xi^{3}-20 D^{3} C v_{\mathrm{t} y}^{2} \xi^{3}-20 A^{2} D C v_{\mathrm{t} y}^{2} \xi^{3}-8 B^{2} D C v_{\mathrm{t} y}^{2} \xi^{3}+16 A^{4} v_{\mathrm{t} z}^{2} \xi^{3}$ $+16 B^{4} v_{\mathrm{t} z}^{2} \xi^{3}+32 A^{2} B^{2} v_{\mathrm{t} z}^{2} \xi^{3}+8 A^{2} D^{2} v_{\mathrm{t} z}^{2} \xi^{3}+8 B^{2} D^{2} v_{\mathrm{t} z}^{2} \xi^{3}-12 A^{2} D C v_{\mathrm{t} z}^{2} \xi^{3}-12 B^{2} D C v_{\mathrm{t} z}^{2} \xi^{3}+40 A B^{3} v_{\mathrm{r} x} v_{\mathrm{r} y} \xi^{3}$ $+24 A B D^{2} v_{\mathrm{r} x} v_{\mathrm{r} y} \xi^{3}+40 A^{3} B v_{\mathrm{r} x} v_{\mathrm{r} y} \xi^{3}+24 A B D C v_{\mathrm{r} x} v_{\mathrm{r} y} \xi^{3}+8 A D^{3} v_{\mathrm{r} x} v_{\mathrm{r} z} \xi^{3}+24 A^{3} D v_{\mathrm{r} x} v_{\mathrm{r} z} \xi^{3}+24 A B^{2} D v_{\mathrm{r} x} v_{\mathrm{r} z} \xi^{3}$ $+8 A^{3} C v_{\mathrm{r} x} v_{\mathrm{r} z} \xi^{3}+8 A B^{2} C v_{\mathrm{r} x} v_{\mathrm{r} z} \xi^{3}+32 A D^{2} C v_{\mathrm{r} x} v_{\mathrm{r} z} \xi^{3}+8 B D^{3} v_{\mathrm{r} y} v_{\mathrm{r} z} \xi^{3}+24 B^{3} D v_{\mathrm{r} y} v_{\mathrm{r} z} \xi^{3}+24 A^{2} B D v_{\mathrm{r} y} v_{\mathrm{r} z} \xi^{3}$ 


$$
\begin{aligned}
& +8 B^{3} C v_{\mathrm{r} y} v_{\mathrm{r} z} \xi^{3}+32 B D^{2} C v_{\mathrm{r} y} v_{\mathrm{r} z} \xi^{3}+8 A^{2} B C v_{\mathrm{r} y} v_{\mathrm{r} z} \xi^{3}-8 D^{3} C v_{\mathrm{r} x} v_{\mathrm{t} x} \xi^{3}-8 B^{2} D C v_{\mathrm{r} x} v_{\mathrm{t} x} \xi^{3}+8 A B D C v_{\mathrm{r} y} v_{\mathrm{t} x} \xi^{3} \\
& +8 A D^{2} C v_{\mathrm{r} z} v_{\mathrm{t} x} \xi^{3}+8 A B D C v_{\mathrm{r} x} v_{\mathrm{t} y} \xi^{3}-8 D^{3} C v_{\mathrm{r} y} v_{\mathrm{t} y} \xi^{3}-8 A^{2} D C v_{\mathrm{r} y} v_{\mathrm{t} y} \xi^{3}+8 B D^{2} C v_{\mathrm{r} z} v_{\mathrm{t} y} \xi^{3}-40 A B^{3} v_{\mathrm{t} x} v_{\mathrm{t} y} \xi^{3} \\
& -24 A B D^{2} v_{\mathrm{t} x} v_{\mathrm{t} y} \xi^{3}-40 A^{3} B v_{\mathrm{t} x} v_{\mathrm{t} y} \xi^{3}+24 A B D C v_{\mathrm{t} x} v_{\mathrm{t} y} \xi^{3}-8 A D^{2} C v_{\mathrm{r} x} v_{\mathrm{t} z} \xi^{3}-8 B D^{2} C v_{\mathrm{r} y} v_{\mathrm{t} z} \xi^{3}+8 A^{2} D C v_{\mathrm{r} z} v_{\mathrm{t} z} \xi^{3} \\
& +8 B^{2} D C v_{\mathrm{r} z} v_{\mathrm{t} z} \xi^{3}+8 A D^{3} v_{\mathrm{t} x} v_{\mathrm{t} z} \xi^{3}+24 A^{3} D v_{\mathrm{t} x} v_{\mathrm{t} z} \xi^{3}+24 A B^{2} D v_{\mathrm{t} x} v_{\mathrm{t} z} \xi^{3}-8 A^{3} C v_{\mathrm{t} x} v_{\mathrm{t} z} \xi^{3}-8 A B^{2} C v_{\mathrm{t} x} v_{\mathrm{t} z} \xi^{3} \\
& -32 A D^{2} C v_{\mathrm{t} x} v_{\mathrm{t} z} \xi^{3}+8 B D^{3} v_{\mathrm{t} y} v_{\mathrm{t} z} \xi^{3}+24 B^{3} D v_{\mathrm{t} y} v_{\mathrm{t} z} \xi^{3}+24 A^{2} B D v_{\mathrm{t} y} v_{\mathrm{t} z} \xi^{3}-8 B^{3} C v_{\mathrm{t} y} v_{\mathrm{t} z} \xi^{3}-32 B D^{2} C v_{\mathrm{t} y} v_{\mathrm{t} z} \xi^{3} \\
& -8 A^{2} B C v_{\mathrm{t} y} v_{\mathrm{t} z} \xi^{3}+4 B^{4} v_{\mathrm{r} x}^{2} \xi+4 D^{4} v_{\mathrm{r} x}^{2} \xi+4 A^{2} B^{2} v_{\mathrm{r} x}^{2} \xi+4 A^{2} D^{2} v_{\mathrm{r} x}^{2} \xi+8 B^{2} D^{2} v_{\mathrm{r} x}^{2} \xi+4 A^{4} v_{\mathrm{r} y}^{2} \xi+4 D^{4} v_{\mathrm{r} y}^{2} \xi+4 A^{2} B^{2} v_{\mathrm{r} y}^{2} \xi \\
& +8 A^{2} D^{2} v_{\mathrm{r} y}^{2} \xi+4 B^{2} D^{2} v_{\mathrm{r} y}^{2} \xi+4 A^{4} v_{\mathrm{r} z}^{2} \xi+4 B^{4} v_{\mathrm{r} z}^{2} \xi+8 A^{2} B^{2} v_{\mathrm{r} z}^{2} \xi+4 A^{2} D^{2} v_{\mathrm{r} z}^{2} \xi+4 B^{2} D^{2} v_{\mathrm{r} z}^{2} \xi-4 B^{4} v_{\mathrm{t} x}^{2} \xi \\
& -4 D^{4} v_{\mathrm{t} x}^{2} \xi-4 A^{2} B^{2} v_{\mathrm{tx}}^{2} \xi-4 A^{2} D^{2} v_{\mathrm{t} x}^{2} \xi-8 B^{2} D^{2} v_{\mathrm{t} x}^{2} \xi-4 A^{4} v_{\mathrm{t} y}^{2} \xi-4 D^{4} v_{\mathrm{t} y}^{2} \xi-4 A^{2} B^{2} v_{\mathrm{t} y}^{2} \xi-8 A^{2} D^{2} v_{\mathrm{t} y}^{2} \xi-4 B^{2} D^{2} v_{\mathrm{t} y}^{2} \xi \\
& -4 A^{4} v_{\mathrm{t} z}^{2} \xi-4 B^{4} v_{\mathrm{t} z}^{2} \xi-8 A^{2} B^{2} v_{\mathrm{t} z}^{2} \xi-4 A^{2} D^{2} v_{\mathrm{t} z}^{2} \xi-4 B^{2} D^{2} v_{\mathrm{t} z}^{2} \xi-8 A B^{3} v_{\mathrm{r} x} v_{\mathrm{r} y} \xi-8 A B D^{2} v_{\mathrm{r} x} v_{\mathrm{r} y} \xi-8 A^{3} B v_{\mathrm{r} x} v_{\mathrm{r} y} \xi \\
& -8 A D^{3} v_{\mathrm{r} x} v_{\mathrm{r} z} \xi-8 A^{3} D v_{\mathrm{r} x} v_{\mathrm{r} z} \xi-8 A B^{2} D v_{\mathrm{r} x} v_{\mathrm{r} z} \xi-8 B D^{3} v_{\mathrm{r} y} v_{\mathrm{r} z} \xi-8 B^{3} D v_{\mathrm{r} y} v_{\mathrm{r} z} \xi-8 A^{2} B D v_{\mathrm{r} y} v_{\mathrm{r} z} \xi+8 A B^{3} v_{\mathrm{t} x} v_{\mathrm{t} y} \xi \\
& +8 A B D^{2} v_{\mathrm{t} x} v_{\mathrm{t} y} \xi+8 A^{3} B v_{\mathrm{t} x} v_{\mathrm{t} y} \xi-8 A D^{3} v_{\mathrm{t} x} v_{\mathrm{t} z} \xi-8 A^{3} D v_{\mathrm{t} x} v_{\mathrm{t} z} \xi-8 A B^{2} D v_{\mathrm{t} x} v_{\mathrm{t} z} \xi-8 B D^{3} v_{\mathrm{t} y} v_{\mathrm{t} z} \xi-8 B^{3} D v_{\mathrm{t} y} v_{\mathrm{t} z} \xi \\
& \left.-8 A^{2} B D v_{\mathrm{ty}} v_{\mathrm{t} z} \xi\right) \eta^{3}
\end{aligned}
$$


$+\left(B^{4} v_{\mathrm{r} x}^{2} \xi^{10}+C^{4} v_{\mathrm{r} x}^{2} \xi^{10}+A^{2} B^{2} v_{\mathrm{r} x}^{2} \xi^{10}+A^{2} C^{2} v_{\mathrm{r} x}^{2} \xi^{10}+2 B^{2} C^{2} v_{\mathrm{r} x}^{2} \xi^{10}+A^{4} v_{\mathrm{r} y}^{2} \xi^{10}+C^{4} v_{\mathrm{r} y}^{2} \xi^{10}+A^{2} B^{2} v_{\mathrm{r} y}^{2} \xi^{10}\right.$ $+2 A^{2} C^{2} v_{\mathrm{r} y}^{2} \xi^{10}+B^{2} C^{2} v_{\mathrm{r} y}^{2} \xi^{10}+A^{4} v_{\mathrm{r} z}^{2} \xi^{10}+B^{4} v_{\mathrm{r} z}^{2} \xi^{10}+2 A^{2} B^{2} v_{\mathrm{r} z}^{2} \xi^{10}+A^{2} C^{2} v_{\mathrm{r} z}^{2} \xi^{10}+B^{2} C^{2} v_{\mathrm{r} z}^{2} \xi^{10}+B^{4} v_{\mathrm{t} x}^{2} \xi^{10}$ $+C^{4} v_{\mathrm{t} x}^{2} \xi^{10}+A^{2} B^{2} v_{\mathrm{t} x}^{2} \xi^{10}+A^{2} C^{2} v_{\mathrm{t} x}^{2} \xi^{10}+2 B^{2} C^{2} v_{\mathrm{t} x}^{2} \xi^{10}+A^{4} v_{\mathrm{t} y}^{2} \xi^{10}+C^{4} v_{\mathrm{t} y}^{2} \xi^{10}+A^{2} B^{2} v_{\mathrm{t} y}^{2} \xi^{10}+2 A^{2} C^{2} v_{\mathrm{t} y}^{2} \xi^{10}$ $+B^{2} C^{2} v_{\mathrm{t} y}^{2} \xi^{10}+A^{4} v_{\mathrm{t} z}^{2} \xi^{10}+B^{4} v_{\mathrm{t} z}^{2} \xi^{10}+2 A^{2} B^{2} v_{\mathrm{t} z}^{2} \xi^{10}+A^{2} C^{2} v_{\mathrm{t} z}^{2} \xi^{10}+B^{2} C^{2} v_{\mathrm{t} z}^{2} \xi^{10}-2 A B^{3} v_{\mathrm{r} x} v_{\mathrm{r} y} \xi^{10}$ $-2 A B C^{2} v_{\mathrm{r} x} v_{\mathrm{r} y} \xi^{10}-2 A^{3} B v_{\mathrm{r} x} v_{\mathrm{r} y} \xi^{10}-2 A C^{3} v_{\mathrm{r} x} v_{\mathrm{r} z} \xi^{10}-2 A^{3} C v_{\mathrm{r} x} v_{\mathrm{r} z} \xi^{10}-2 A B^{2} C v_{\mathrm{r} x} v_{\mathrm{r} z} \xi^{10}-2 B C^{3} v_{\mathrm{r} y} v_{\mathrm{r} z} \xi^{10}$ $-2 B^{3} C v_{\mathrm{r} y} v_{\mathrm{r} z} \xi^{10}-2 A^{2} B C v_{\mathrm{r} y} v_{\mathrm{r} z} \xi^{10}+2 B^{4} v_{\mathrm{r} x} v_{\mathrm{t} x} \xi^{10}+2 C^{4} v_{\mathrm{r} x} v_{\mathrm{t} x} \xi^{10}+2 A^{2} B^{2} v_{\mathrm{r} x} v_{\mathrm{t} x} \xi^{10}+2 A^{2} C^{2} v_{\mathrm{r} x} v_{\mathrm{t} x} \xi^{10}$ $+4 B^{2} C^{2} v_{\mathrm{r} x} v_{\mathrm{t} x} \xi^{10}-2 A B^{3} v_{\mathrm{r} y} v_{\mathrm{t} x} \xi^{10}-2 A B C^{2} v_{\mathrm{r} y} v_{\mathrm{t} x} \xi^{10}-2 A^{3} B v_{\mathrm{r} y} v_{\mathrm{t} x} \xi^{10}-2 A C^{3} v_{\mathrm{r} z} v_{\mathrm{t} x} \xi^{10}-2 A^{3} C v_{\mathrm{r} z} v_{\mathrm{t} x} \xi^{10}$ $-2 A B^{2} C v_{\mathrm{r} z} v_{\mathrm{t} x} \xi^{10}-2 A B^{3} v_{\mathrm{r} x} v_{\mathrm{t} y} \xi^{10}-2 A B C^{2} v_{\mathrm{r} x} v_{\mathrm{t} y} \xi^{10}-2 A^{3} B v_{\mathrm{r} x} v_{\mathrm{t} y} \xi^{10}+2 A^{4} v_{\mathrm{r} y} v_{\mathrm{t} y} \xi^{10}+2 C^{4} v_{\mathrm{r} y} v_{\mathrm{t} y} \xi^{10}$ $+2 A^{2} B^{2} v_{\mathrm{r} y} v_{\mathrm{t} y} \xi^{10}+4 A^{2} C^{2} v_{\mathrm{r} y} v_{\mathrm{t} y} \xi^{10}+2 B^{2} C^{2} v_{\mathrm{r} y} v_{\mathrm{t} y} \xi^{10}-2 B C^{3} v_{\mathrm{r} z} v_{\mathrm{t} y} \xi^{10}-2 B^{3} C v_{\mathrm{r} z} v_{\mathrm{t} y} \xi^{10}-2 A^{2} B C v_{\mathrm{r} z} v_{\mathrm{t} y} \xi^{10}$ $-2 A B^{3} v_{\mathrm{t} x} v_{\mathrm{t} y} \xi^{10}-2 A B C^{2} v_{\mathrm{t} x} v_{\mathrm{t} y} \xi^{10}-2 A^{3} B v_{\mathrm{t} x} v_{\mathrm{t} y} \xi^{10}-2 A C^{3} v_{\mathrm{r} x} v_{\mathrm{t} z} \xi^{10}-2 A^{3} C v_{\mathrm{r} x} v_{\mathrm{t} z} \xi^{10}-2 A B^{2} C v_{\mathrm{r} x} v_{\mathrm{t} z} \xi^{10}$ $-2 B C^{3} v_{\mathrm{r} y} v_{\mathrm{t} z} \xi^{10}-2 B^{3} C v_{\mathrm{r} y} v_{\mathrm{t} z} \xi^{10}-2 A^{2} B C v_{\mathrm{r} y} v_{\mathrm{t} z} \xi^{10}+2 A^{4} v_{\mathrm{r} z} v_{\mathrm{t} z} \xi^{10}+2 B^{4} v_{\mathrm{r} z} v_{\mathrm{t} z} \xi^{10}+4 A^{2} B^{2} v_{\mathrm{r} z} v_{\mathrm{t} z} \xi^{10}$ $+2 A^{2} C^{2} v_{\mathrm{r} z} v_{\mathrm{t} z} \xi^{10}+2 B^{2} C^{2} v_{\mathrm{r} z} v_{\mathrm{t} z} \xi^{10}-2 A C^{3} v_{\mathrm{t} x} v_{\mathrm{t} z} \xi^{10}-2 A^{3} C v_{\mathrm{t} x} v_{\mathrm{t} z} \xi^{10}-2 A B^{2} C v_{\mathrm{t} x} v_{\mathrm{t} z} \xi^{10}-2 B C^{3} v_{\mathrm{t} y} v_{\mathrm{t} z} \xi^{10}$ $-2 B^{3} C v_{\mathrm{t} y} v_{\mathrm{t} z} \xi^{10}-2 A^{2} B C v_{\mathrm{t} y} v_{\mathrm{t} z} \xi^{10}-10 B^{4} v_{\mathrm{r} x}^{2} \xi^{8}-10 D C^{3} v_{\mathrm{r} x}^{2} \xi^{8}-10 A^{2} B^{2} v_{\mathrm{r} x}^{2} \xi^{8}-7 A^{2} C^{2} v_{\mathrm{r} x}^{2} \xi^{8}-10 B^{2} C^{2} v_{\mathrm{r} x}^{2} \xi^{8}$ $-2 A^{2} D C v_{\mathrm{r} x}^{2} \xi^{8}-10 B^{2} D C v_{\mathrm{r} x}^{2} \xi^{8}-10 A^{4} v_{\mathrm{r} y}^{2} \xi^{8}-10 D C^{3} v_{\mathrm{r} y}^{2} \xi^{8}-10 A^{2} B^{2} v_{\mathrm{r} y}^{2} \xi^{8}-10 A^{2} C^{2} v_{\mathrm{r} y}^{2} \xi^{8}-7 B^{2} C^{2} v_{\mathrm{r} y}^{2} \xi^{8}$ $-10 A^{2} D C v_{\mathrm{r} y}^{2} \xi^{8}-2 B^{2} D C v_{\mathrm{r} y}^{2} \xi^{8}-9 A^{4} v_{\mathrm{r} z}^{2} \xi^{8}-9 B^{4} v_{\mathrm{r} z}^{2} \xi^{8}-18 A^{2} B^{2} v_{\mathrm{r} z}^{2} \xi^{8}-2 A^{2} C^{2} v_{\mathrm{r} z}^{2} \xi^{8}-2 B^{2} C^{2} v_{\mathrm{r} z}^{2} \xi^{8}-8 A^{2} D C v_{\mathrm{r} z}^{2} \xi^{8}$ $-8 B^{2} D C v_{\mathrm{r} z}^{2} \xi^{8}-10 B^{4} v_{\mathrm{t} x}^{2} \xi^{8}+10 D C^{3} v_{\mathrm{t} x}^{2} \xi^{8}-10 A^{2} B^{2} v_{\mathrm{t} x}^{2} \xi^{8}-7 A^{2} C^{2} v_{\mathrm{t} x}^{2} \xi^{8}-10 B^{2} C^{2} v_{\mathrm{t} x}^{2} \xi^{8}+2 A^{2} D C v_{\mathrm{t} x}^{2} \xi^{8}$ $+10 B^{2} D C v_{\mathrm{t} x}^{2} \xi^{8}-10 A^{4} v_{\mathrm{t} y}^{2} \xi^{8}+10 D C^{3} v_{\mathrm{t} y}^{2} \xi^{8}-10 A^{2} B^{2} v_{\mathrm{t} y}^{2} \xi^{8}-10 A^{2} C^{2} v_{\mathrm{t} y}^{2} \xi^{8}-7 B^{2} C^{2} v_{\mathrm{t} y}^{2} \xi^{8}+10 A^{2} D C v_{\mathrm{t} y}^{2} \xi^{8}$ $+2 B^{2} D C v_{\mathrm{t} y}^{2} \xi^{8}-9 A^{4} v_{\mathrm{t} z}^{2} \xi^{8}-9 B^{4} v_{\mathrm{t} z}^{2} \xi^{8}-18 A^{2} B^{2} v_{\mathrm{t} z}^{2} \xi^{8}-2 A^{2} C^{2} v_{\mathrm{t} z}^{2} \xi^{8}-2 B^{2} C^{2} v_{\mathrm{t} z}^{2} \xi^{8}+8 A^{2} D C v_{\mathrm{t} z}^{2} \xi^{8}+8 B^{2} D C v_{\mathrm{t} z}^{2} \xi^{8}$ $+20 A B^{3} v_{\mathrm{r} x} v_{\mathrm{r} y} \xi^{8}+6 A B C^{2} v_{\mathrm{r} x} v_{\mathrm{r} y} \xi^{8}+20 A^{3} B v_{\mathrm{r} x} v_{\mathrm{r} y} \xi^{8}+16 A B D C v_{\mathrm{r} x} v_{\mathrm{r} y} \xi^{8}+2 A C^{3} v_{\mathrm{r} x} v_{\mathrm{r} z} \xi^{8}+18 A D C^{2} v_{\mathrm{r} x} v_{\mathrm{r} z} \xi^{8}$ $+2 A^{3} D v_{\mathrm{r} x} v_{\mathrm{r} z} \xi^{8}+2 A B^{2} D v_{\mathrm{r} x} v_{\mathrm{r} z} \xi^{8}+16 A^{3} C v_{\mathrm{r} x} v_{\mathrm{r} z} \xi^{8}+16 A B^{2} C v_{\mathrm{r} x} v_{\mathrm{r} z} \xi^{8}+2 B C^{3} v_{\mathrm{r} y} v_{\mathrm{r} z} \xi^{8}+18 B D C^{2} v_{\mathrm{r} y} v_{\mathrm{r} z} \xi^{8}$ $+2 B^{3} D v_{\mathrm{r} y} v_{\mathrm{r} z} \xi^{8}+2 A^{2} B D v_{\mathrm{r} y} v_{\mathrm{r} z} \xi^{8}+16 B^{3} C v_{\mathrm{r} y} v_{\mathrm{r} z} \xi^{8}+16 A^{2} B C v_{\mathrm{r} y} v_{\mathrm{r} z} \xi^{8}-4 B^{4} v_{\mathrm{r} x} v_{\mathrm{t} x} \xi^{8}-4 A^{2} B^{2} v_{\mathrm{r} x} v_{\mathrm{t} x} \xi^{8}$ $+2 A^{2} C^{2} v_{\mathrm{r} x} v_{\mathrm{t} x} \xi^{8}-4 B^{2} C^{2} v_{\mathrm{r} x} v_{\mathrm{t} x} \xi^{8}+4 A B^{3} v_{\mathrm{r} y} v_{\mathrm{t} x} \xi^{8}+6 A B C^{2} v_{\mathrm{r} y} v_{\mathrm{t} x} \xi^{8}+4 A^{3} B v_{\mathrm{r} y} v_{\mathrm{t} x} \xi^{8}+2 A C^{3} v_{\mathrm{r} z} v_{\mathrm{t} x} \xi^{8}$ $-2 A D C^{2} v_{\mathrm{r} z} v_{\mathrm{t} x} \xi^{8}-2 A^{3} D v_{\mathrm{r} z} v_{\mathrm{t} x} \xi^{8}-2 A B^{2} D v_{\mathrm{r} z} v_{\mathrm{t} x} \xi^{8}+4 A B^{3} v_{\mathrm{r} x} v_{\mathrm{t} y} \xi^{8}+6 A B C^{2} v_{\mathrm{r} x} v_{\mathrm{t} y} \xi^{8}+4 A^{3} B v_{\mathrm{r} x} v_{\mathrm{t} y} \xi^{8}$ $-4 A^{4} v_{\mathrm{r} y} v_{\mathrm{t} y} \xi^{8}-4 A^{2} B^{2} v_{\mathrm{r} y} v_{\mathrm{t} y} \xi^{8}-4 A^{2} C^{2} v_{\mathrm{r} y} v_{\mathrm{t} y} \xi^{8}+2 B^{2} C^{2} v_{\mathrm{r} y} v_{\mathrm{t} y} \xi^{8}+2 B C^{3} v_{\mathrm{r} z} v_{\mathrm{t} y} \xi^{8}-2 B D C^{2} v_{\mathrm{r} z} v_{\mathrm{t} y} \xi^{8}$ $-2 B^{3} D v_{\mathrm{r} z} v_{\mathrm{t} y} \xi^{8}-2 A^{2} B D v_{\mathrm{r} z} v_{\mathrm{t} y} \xi^{8}+20 A B^{3} v_{\mathrm{t} x} v_{\mathrm{t} y} \xi^{8}+6 A B C^{2} v_{\mathrm{t} x} v_{\mathrm{t} y} \xi^{8}+20 A^{3} B v_{\mathrm{t} x} v_{\mathrm{t} y} \xi^{8}-16 A B D C v_{\mathrm{t} x} v_{\mathrm{t} y} \xi^{8}$ $+2 A C^{3} v_{\mathrm{r} x} v_{\mathrm{t} z} \xi^{8}+2 A D C^{2} v_{\mathrm{r} x} v_{\mathrm{t} z} \xi^{8}+2 A^{3} D v_{\mathrm{r} x} v_{\mathrm{t} z} \xi^{8}+2 A B^{2} D v_{\mathrm{r} x} v_{\mathrm{t} z} \xi^{8}+2 B C^{3} v_{\mathrm{r} y} v_{\mathrm{t} z} \xi^{8}+2 B D C^{2} v_{\mathrm{r} y} v_{\mathrm{t} z} \xi^{8}$ $+2 B^{3} D v_{\mathrm{r} y} v_{\mathrm{t} z} \xi^{8}+2 A^{2} B D v_{\mathrm{r} y} v_{\mathrm{t} z} \xi^{8}-2 A^{4} v_{\mathrm{r} z} v_{\mathrm{t} z} \xi^{8}-2 B^{4} v_{\mathrm{r} z} v_{\mathrm{t} z} \xi^{8}-4 A^{2} B^{2} v_{\mathrm{r} z} v_{\mathrm{t} z} \xi^{8}-4 A^{2} C^{2} v_{\mathrm{r} z} v_{\mathrm{t} z} \xi^{8}-4 B^{2} C^{2} v_{\mathrm{r} z} v_{\mathrm{t} z} \xi^{8}$ $+2 A C^{3} v_{\mathrm{t} x} v_{\mathrm{t} z} \xi^{8}-18 A D C^{2} v_{\mathrm{t} x} v_{\mathrm{t} z} \xi^{8}-2 A^{3} D v_{\mathrm{t} x} v_{\mathrm{t} z} \xi^{8}-2 A B^{2} D v_{\mathrm{t} x} v_{\mathrm{t} z} \xi^{8}+16 A^{3} C v_{\mathrm{t} x} v_{\mathrm{t} z} \xi^{8}+16 A B^{2} C v_{\mathrm{t} x} v_{\mathrm{t} z} \xi^{8}$ $+2 B C^{3} v_{\mathrm{t} y} v_{\mathrm{t} z} \xi^{8}-18 B D C^{2} v_{\mathrm{t} y} v_{\mathrm{t} z} \xi^{8}-2 B^{3} D v_{\mathrm{t} y} v_{\mathrm{t} z} \xi^{8}-2 A^{2} B D v_{\mathrm{t} y} v_{\mathrm{t} z} \xi^{8}+16 B^{3} C v_{\mathrm{t} y} v_{\mathrm{t} z} \xi^{8}+16 A^{2} B C v_{\mathrm{t} y} v_{\mathrm{t} z} \xi^{8}$ $+23 B^{4} v_{\mathrm{r} x}^{2} \xi^{6}+23 A^{2} B^{2} v_{\mathrm{r} x}^{2} \xi^{6}+A^{2} D^{2} v_{\mathrm{r} x}^{2} \xi^{6}+8 B^{2} D^{2} v_{\mathrm{r} x}^{2} \xi^{6}+6 A^{2} C^{2} v_{\mathrm{r} x}^{2} \xi^{6}+8 B^{2} C^{2} v_{\mathrm{r} x}^{2} \xi^{6}+23 D^{2} C^{2} v_{\mathrm{r} x}^{2} \xi^{6}$ $+14 A^{2} D C v_{\mathrm{r} x}^{2} \xi^{6}+30 B^{2} D C v_{\mathrm{r} x}^{2} \xi^{6}+23 A^{4} v_{\mathrm{r} y}^{2} \xi^{6}+23 A^{2} B^{2} v_{\mathrm{r} y}^{2} \xi^{6}+8 A^{2} D^{2} v_{\mathrm{r} y}^{2} \xi^{6}+B^{2} D^{2} v_{\mathrm{r} y}^{2} \xi^{6}+8 A^{2} C^{2} v_{\mathrm{r} y}^{2} \xi^{6}$ $+6 B^{2} C^{2} v_{\mathrm{r} y}^{2} \xi^{6}+23 D^{2} C^{2} v_{\mathrm{r} y}^{2} \xi^{6}+30 A^{2} D C v_{\mathrm{r} y}^{2} \xi^{6}+14 B^{2} D C v_{\mathrm{r} y}^{2} \xi^{6}+21 A^{4} v_{\mathrm{r} z}^{2} \xi^{6}+21 B^{4} v_{\mathrm{r} z}^{2} \xi^{6}+42 A^{2} B^{2} v_{\mathrm{r} z}^{2} \xi^{6}$ $+6 A^{2} D^{2} v_{\mathrm{r} z}^{2} \xi^{6}+6 B^{2} D^{2} v_{\mathrm{r} z}^{2} \xi^{6}+A^{2} C^{2} v_{\mathrm{r} z}^{2} \xi^{6}+B^{2} C^{2} v_{\mathrm{r} z}^{2} \xi^{6}+16 A^{2} D C v_{\mathrm{r} z}^{2} \xi^{6}+16 B^{2} D C v_{\mathrm{r} z}^{2} \xi^{6}+23 B^{4} v_{\mathrm{t} x}^{2} \xi^{6}+23 A^{2} B^{2} v_{\mathrm{t} x}^{2} \xi^{6}$ $+A^{2} D^{2} v_{\mathrm{t} x}^{2} \xi^{6}+8 B^{2} D^{2} v_{\mathrm{t} x}^{2} \xi^{6}+6 A^{2} C^{2} v_{\mathrm{t} x}^{2} \xi^{6}+8 B^{2} C^{2} v_{\mathrm{t} x}^{2} \xi^{6}+23 D^{2} C^{2} v_{\mathrm{t} x}^{2} \xi^{6}-14 A^{2} D C v_{\mathrm{t} x}^{2} \xi^{6}-30 B^{2} D C v_{\mathrm{t} x}^{2} \xi^{6}$ $+23 A^{4} v_{\mathrm{t} y}^{2} \xi^{6}+23 A^{2} B^{2} v_{\mathrm{t} y}^{2} \xi^{6}+8 A^{2} D^{2} v_{\mathrm{t} y}^{2} \xi^{6}+B^{2} D^{2} v_{\mathrm{t} y}^{2} \xi^{6}+8 A^{2} C^{2} v_{\mathrm{t} y}^{2} \xi^{6}+6 B^{2} C^{2} v_{\mathrm{t} y}^{2} \xi^{6}+23 D^{2} C^{2} v_{\mathrm{t} y}^{2} \xi^{6}$ $-30 A^{2} D C v_{\mathrm{t} y}^{2} \xi^{6}-14 B^{2} D C v_{\mathrm{t} y}^{2} \xi^{6}+21 A^{4} v_{\mathrm{t} z}^{2} \xi^{6}+21 B^{4} v_{\mathrm{t} z}^{2} \xi^{6}+42 A^{2} B^{2} v_{\mathrm{t} z}^{2} \xi^{6}+6 A^{2} D^{2} v_{\mathrm{t} z}^{2} \xi^{6}+6 B^{2} D^{2} v_{\mathrm{t} z}^{2} \xi^{6}+A^{2} C^{2} v_{\mathrm{t} z}^{2} \xi^{6}$ $+B^{2} C^{2} v_{\mathrm{t} z}^{2} \xi^{6}-16 A^{2} D C v_{\mathrm{t} z}^{2} \xi^{6}-16 B^{2} D C v_{\mathrm{t} z}^{2} \xi^{6}-46 A B^{3} v_{\mathrm{r} x} v_{\mathrm{r} y} \xi^{6}-14 A B D^{2} v_{\mathrm{r} x} v_{\mathrm{r} y} \xi^{6}-4 A B C^{2} v_{\mathrm{r} x} v_{\mathrm{r} y} \xi^{6}-46 A^{3} B v_{\mathrm{r} x} v_{\mathrm{r} y} \xi^{6}$ $-32 A B D C v_{\mathrm{r} x} v_{\mathrm{r} y} \xi^{6}-18 A D C^{2} v_{\mathrm{r} x} v_{\mathrm{r} z} \xi^{6}-16 A^{3} D v_{\mathrm{r} x} v_{\mathrm{r} z} \xi^{6}-16 A B^{2} D v_{\mathrm{r} x} v_{\mathrm{r} z} \xi^{6}-26 A^{3} C v_{\mathrm{r} x} v_{\mathrm{r} z} \xi^{6}-26 A B^{2} C v_{\mathrm{r} x} v_{\mathrm{r} z} \xi^{6}$ $-28 A D^{2} C v_{\mathrm{r} x} v_{\mathrm{r} z} \xi^{6}-18 B D C^{2} v_{\mathrm{r} y} v_{\mathrm{r} z} \xi^{6}-16 B^{3} D v_{\mathrm{r} y} v_{\mathrm{r} z} \xi^{6}-16 A^{2} B D v_{\mathrm{r} y} v_{\mathrm{r} z} \xi^{6}-26 B^{3} C v_{\mathrm{r} y} v_{\mathrm{r} z} \xi^{6}-28 B D^{2} C v_{\mathrm{r} y} v_{\mathrm{r} z} \xi^{6}$ $-26 A^{2} B C v_{\mathrm{r} y} v_{\mathrm{r} z} \xi^{6}+6 B^{4} v_{\mathrm{r} x} v_{\mathrm{t} x} \xi^{6}+6 A^{2} B^{2} v_{\mathrm{r} x} v_{\mathrm{t} x} \xi^{6}-2 A^{2} D^{2} v_{\mathrm{r} x} v_{\mathrm{t} x} \xi^{6}-4 A^{2} C^{2} v_{\mathrm{r} x} v_{\mathrm{t} x} \xi^{6}-6 D^{2} C^{2} v_{\mathrm{r} x} v_{\mathrm{t} x} \xi^{6}$ $-6 A B^{3} v_{\mathrm{r} y} v_{\mathrm{t} x} \xi^{6}-2 A B D^{2} v_{\mathrm{r} y} v_{\mathrm{t} x} \xi^{6}-4 A B C^{2} v_{\mathrm{r} y} v_{\mathrm{t} x} \xi^{6}-6 A^{3} B v_{\mathrm{r} y} v_{\mathrm{t} x} \xi^{6}+2 A D C^{2} v_{\mathrm{r} z} v_{\mathrm{t} x} \xi^{6}+6 A^{3} C v_{\mathrm{r} z} v_{\mathrm{t} x} \xi^{6}$ $+6 A B^{2} C v_{\mathrm{r} z} v_{\mathrm{t} x} \xi^{6}+4 A D^{2} C v_{\mathrm{r} z} v_{\mathrm{t} x} \xi^{6}-6 A B^{3} v_{\mathrm{r} x} v_{\mathrm{t} y} \xi^{6}-2 A B D^{2} v_{\mathrm{r} x} v_{\mathrm{t} y} \xi^{6}-4 A B C^{2} v_{\mathrm{r} x} v_{\mathrm{t} y} \xi^{6}-6 A^{3} B v_{\mathrm{r} x} v_{\mathrm{t} y} \xi^{6}$ $+6 A^{4} v_{\mathrm{r} y} v_{\mathrm{t} y} \xi^{6}+6 A^{2} B^{2} v_{\mathrm{r} y} v_{\mathrm{t} y} \xi^{6}-2 B^{2} D^{2} v_{\mathrm{r} y} v_{\mathrm{t} y} \xi^{6}-4 B^{2} C^{2} v_{\mathrm{r} y} v_{\mathrm{t} y} \xi^{6}-6 D^{2} C^{2} v_{\mathrm{r} y} v_{\mathrm{t} y} \xi^{6}+2 B D C^{2} v_{\mathrm{r} z} v_{\mathrm{t} y} \xi^{6}$ $+6 B^{3} C v_{\mathrm{r} z} v_{\mathrm{t} y} \xi^{6}+4 B D^{2} C v_{\mathrm{r} z} v_{\mathrm{t} y} \xi^{6}+6 A^{2} B C v_{\mathrm{r} z} v_{\mathrm{t} y} \xi^{6}-46 A B^{3} v_{\mathrm{t} x} v_{\mathrm{t} y} \xi^{6}-14 A B D^{2} v_{\mathrm{t} x} v_{\mathrm{t} y} \xi^{6}-4 A B C^{2} v_{\mathrm{t} x} v_{\mathrm{t} y} \xi^{6}$ 
$-46 A^{3} B v_{\mathrm{t} x} v_{\mathrm{t} y} \xi^{6}+32 A B D C v_{\mathrm{t} x} v_{\mathrm{t} y} \xi^{6}-2 A D C^{2} v_{\mathrm{r} x} v_{\mathrm{t} z} \xi^{6}+6 A^{3} C v_{\mathrm{r} x} v_{\mathrm{t} z} \xi^{6}+6 A B^{2} C v_{\mathrm{r} x} v_{\mathrm{t} z} \xi^{6}+4 A D^{2} C v_{\mathrm{r} x} v_{\mathrm{t} z} \xi^{6}$ $-2 B D C^{2} v_{\mathrm{r} y} v_{\mathrm{t} z} \xi^{6}+6 B^{3} C v_{\mathrm{r} y} v_{\mathrm{t} z} \xi^{6}+4 B D^{2} C v_{\mathrm{r} y} v_{\mathrm{t} z} \xi^{6}+6 A^{2} B C v_{\mathrm{r} y} v_{\mathrm{t} z} \xi^{6}-6 A^{4} v_{\mathrm{r} z} v_{\mathrm{t} z} \xi^{6}-6 B^{4} v_{\mathrm{r} z} v_{\mathrm{t} z} \xi^{6}$ $-12 A^{2} B^{2} v_{\mathrm{r} z} v_{\mathrm{t} z} \xi^{6}-4 A^{2} D^{2} v_{\mathrm{r} z} v_{\mathrm{t} z} \xi^{6}-4 B^{2} D^{2} v_{\mathrm{r} z} v_{\mathrm{t} z} \xi^{6}+2 A^{2} C^{2} v_{\mathrm{r} z} v_{\mathrm{t} z} \xi^{6}+2 B^{2} C^{2} v_{\mathrm{r} z} v_{\mathrm{t} z} \xi^{6}+18 A D C^{2} v_{\mathrm{t} x} v_{\mathrm{t} z} \xi^{6}$ $+16 A^{3} D v_{\mathrm{t} x} v_{\mathrm{t} z} \xi^{6}+16 A B^{2} D v_{\mathrm{t} x} v_{\mathrm{t} z} \xi^{6}-26 A^{3} C v_{\mathrm{t} x} v_{\mathrm{t} z} \xi^{6}-26 A B^{2} C v_{\mathrm{t} x} v_{\mathrm{t} z} \xi^{6}-28 A D^{2} C v_{\mathrm{t} x} v_{\mathrm{t} z} \xi^{6}+18 B D C^{2} v_{\mathrm{t} y} v_{\mathrm{t} z} \xi^{6}$ $+16 B^{3} D v_{\mathrm{t} y} v_{\mathrm{t} z} \xi^{6}+16 A^{2} B D v_{\mathrm{t} y} v_{\mathrm{t} z} \xi^{6}-26 B^{3} C v_{\mathrm{t} y} v_{\mathrm{t} z} \xi^{6}-28 B D^{2} C v_{\mathrm{t} y} v_{\mathrm{t} z} \xi^{6}-26 A^{2} B C v_{\mathrm{t} y} v_{\mathrm{t} z} \xi^{6}-20 B^{4} v_{\mathrm{r} x}^{2} \xi^{4}$ $-20 A^{2} B^{2} v_{\mathrm{r} x}^{2} \xi^{4}-7 A^{2} D^{2} v_{\mathrm{r} x}^{2} \xi^{4}-20 B^{2} D^{2} v_{\mathrm{r} x}^{2} \xi^{4}-20 D^{3} C v_{\mathrm{r} x}^{2} \xi^{4}-12 A^{2} D C v_{\mathrm{r} x}^{2} \xi^{4}-20 B^{2} D C v_{\mathrm{r} x}^{2} \xi^{4}-20 A^{4} v_{\mathrm{r} y}^{2} \xi^{4}$ $-20 A^{2} B^{2} v_{\mathrm{r} y}^{2} \xi^{4}-20 A^{2} D^{2} v_{\mathrm{r} y}^{2} \xi^{4}-7 B^{2} D^{2} v_{\mathrm{r} y}^{2} \xi^{4}-20 D^{3} C v_{\mathrm{r} y}^{2} \xi^{4}-20 A^{2} D C v_{\mathrm{r} y}^{2} \xi^{4}-12 B^{2} D C v_{\mathrm{r} y}^{2} \xi^{4}-19 A^{4} v_{\mathrm{r} z}^{2} \xi^{4}$ $-19 B^{4} v_{\mathrm{r} z}^{2} \xi^{4}-38 A^{2} B^{2} v_{\mathrm{r} z}^{2} \xi^{4}-12 A^{2} D^{2} v_{\mathrm{r} z}^{2} \xi^{4}-12 B^{2} D^{2} v_{\mathrm{r} z}^{2} \xi^{4}-8 A^{2} D C v_{\mathrm{r} z}^{2} \xi^{4}-8 B^{2} D C v_{\mathrm{r} z}^{2} \xi^{4}-20 B^{4} v_{\mathrm{t} x}^{2} \xi^{4}$ $-20 A^{2} B^{2} v_{\mathrm{t} x}^{2} \xi^{4}-7 A^{2} D^{2} v_{\mathrm{t} x}^{2} \xi^{4}-20 B^{2} D^{2} v_{\mathrm{t} x}^{2} \xi^{4}+20 D^{3} C v_{\mathrm{t} x}^{2} \xi^{4}+12 A^{2} D C v_{\mathrm{t} x}^{2} \xi^{4}+20 B^{2} D C v_{\mathrm{t} x}^{2} \xi^{4}-20 A^{4} v_{\mathrm{t} y}^{2} \xi^{4}$ $-20 A^{2} B^{2} v_{\mathrm{t} y}^{2} \xi^{4}-20 A^{2} D^{2} v_{\mathrm{t} y}^{2} \xi^{4}-7 B^{2} D^{2} v_{\mathrm{t} y}^{2} \xi^{4}+20 D^{3} C v_{\mathrm{t} y}^{2} \xi^{4}+20 A^{2} D C v_{\mathrm{t} y}^{2} \xi^{4}+12 B^{2} D C v_{\mathrm{t} y}^{2} \xi^{4}-19 A^{4} v_{\mathrm{t} z}^{2} \xi^{4}$ $-19 B^{4} v_{\mathrm{t} z}^{2} \xi^{4}-38 A^{2} B^{2} v_{\mathrm{t} z}^{2} \xi^{4}-12 A^{2} D^{2} v_{\mathrm{t} z}^{2} \xi^{4}-12 B^{2} D^{2} v_{\mathrm{t} z}^{2} \xi^{4}+8 A^{2} D C v_{\mathrm{t} z}^{2} \xi^{4}+8 B^{2} D C v_{\mathrm{t} z}^{2} \xi^{4}+40 A B^{3} v_{\mathrm{r} x} v_{\mathrm{r} y} \xi^{4}$ $+26 A B D^{2} v_{\mathrm{r} x} v_{\mathrm{r} y} \xi^{4}+40 A^{3} B v_{\mathrm{r} x} v_{\mathrm{r} y} \xi^{4}+16 A B D C v_{\mathrm{r} x} v_{\mathrm{r} y} \xi^{4}+12 A D^{3} v_{\mathrm{r} x} v_{\mathrm{r} z} \xi^{4}+26 A^{3} D v_{\mathrm{r} x} v_{\mathrm{r} z} \xi^{4}+26 A B^{2} D v_{\mathrm{r} x} v_{\mathrm{r} z} \xi^{4}$ $+12 A^{3} C v_{\mathrm{r} x} v_{\mathrm{r} z} \xi^{4}+12 A B^{2} C v_{\mathrm{r} x} v_{\mathrm{r} z} \xi^{4}+28 A D^{2} C v_{\mathrm{r} x} v_{\mathrm{r} z} \xi^{4}+12 B D^{3} v_{\mathrm{r} y} v_{\mathrm{r} z} \xi^{4}+26 B^{3} D v_{\mathrm{r} y} v_{\mathrm{r} z} \xi^{4}+26 A^{2} B D v_{\mathrm{r} y} v_{\mathrm{r} z} \xi^{4}$ $+12 B^{3} C v_{\mathrm{r} y} v_{\mathrm{r} z} \xi^{4}+28 B D^{2} C v_{\mathrm{r} y} v_{\mathrm{r} z} \xi^{4}+12 A^{2} B C v_{\mathrm{r} y} v_{\mathrm{r} z} \xi^{4}-8 B^{4} v_{\mathrm{r} x} v_{\mathrm{t} x} \xi^{4}-8 A^{2} B^{2} v_{\mathrm{r} x} v_{\mathrm{t} x} \xi^{4}-2 A^{2} D^{2} v_{\mathrm{r} x} v_{\mathrm{t} x} \xi^{4}$ $-8 B^{2} D^{2} v_{\mathrm{r} x} v_{\mathrm{t} x} \xi^{4}+8 A B^{3} v_{\mathrm{r} y} v_{\mathrm{t} x} \xi^{4}+6 A B D^{2} v_{\mathrm{r} y} v_{\mathrm{t} x} \xi^{4}+8 A^{3} B v_{\mathrm{r} y} v_{\mathrm{t} x} \xi^{4}+4 A D^{3} v_{\mathrm{r} z} v_{\mathrm{t} x} \xi^{4}+6 A^{3} D v_{\mathrm{r} z} v_{\mathrm{t} x} \xi^{4}$ $+6 A B^{2} D v_{\mathrm{r} z} v_{\mathrm{t} x} \xi^{4}-4 A^{3} C v_{\mathrm{r} z} v_{\mathrm{t} x} \xi^{4}-4 A B^{2} C v_{\mathrm{r} z} v_{\mathrm{t} x} \xi^{4}-4 A D^{2} C v_{\mathrm{r} z} v_{\mathrm{t} x} \xi^{4}+8 A B^{3} v_{\mathrm{r} x} v_{\mathrm{t} y} \xi^{4}+6 A B D^{2} v_{\mathrm{r} x} v_{\mathrm{t} y} \xi^{4}$ $+8 A^{3} B v_{\mathrm{r} x} v_{\mathrm{t} y} \xi^{4}-8 A^{4} v_{\mathrm{r} y} v_{\mathrm{t} y} \xi^{4}-8 A^{2} B^{2} v_{\mathrm{r} y} v_{\mathrm{t} y} \xi^{4}-8 A^{2} D^{2} v_{\mathrm{r} y} v_{\mathrm{t} y} \xi^{4}-2 B^{2} D^{2} v_{\mathrm{r} y} v_{\mathrm{t} y} \xi^{4}+4 B D^{3} v_{\mathrm{r} z} v_{\mathrm{t} y} \xi^{4}+6 B^{3} D v_{\mathrm{r} z} v_{\mathrm{t} y} \xi^{4}$ $+6 A^{2} B D v_{\mathrm{r} z} v_{\mathrm{t} y} \xi^{4}-4 B^{3} C v_{\mathrm{r} z} v_{\mathrm{t} y} \xi^{4}-4 B D^{2} C v_{\mathrm{r} z} v_{\mathrm{t} y} \xi^{4}-4 A^{2} B C v_{\mathrm{r} z} v_{\mathrm{t} y} \xi^{4}+40 A B^{3} v_{\mathrm{t} x} v_{\mathrm{t} y} \xi^{4}+26 A B D^{2} v_{\mathrm{t} x} v_{\mathrm{t} y} \xi^{4}$ $+40 A^{3} B v_{\mathrm{t} x} v_{\mathrm{t} y} \xi^{4}-16 A B D C v_{\mathrm{t} x} v_{\mathrm{t} y} \xi^{4}-4 A D^{3} v_{\mathrm{r} x} v_{\mathrm{t} z} \xi^{4}-6 A^{3} D v_{\mathrm{r} x} v_{\mathrm{t} z} \xi^{4}-6 A B^{2} D v_{\mathrm{r} x} v_{\mathrm{t} z} \xi^{4}-4 A^{3} C v_{\mathrm{r} x} v_{\mathrm{t} z} \xi^{4}$ $-4 A B^{2} C v_{\mathrm{r} x} v_{\mathrm{t} z} \xi^{4}-4 A D^{2} C v_{\mathrm{r} x} v_{\mathrm{t} z} \xi^{4}-4 B D^{3} v_{\mathrm{r} y} v_{\mathrm{t} z} \xi^{4}-6 B^{3} D v_{\mathrm{r} y} v_{\mathrm{t} z} \xi^{4}-6 A^{2} B D v_{\mathrm{r} y} v_{\mathrm{t} z} \xi^{4}-4 B^{3} C v_{\mathrm{r} y} v_{\mathrm{t} z} \xi^{4}$ $-4 B D^{2} C v_{\mathrm{r} y} v_{\mathrm{t} z} \xi^{4}-4 A^{2} B C v_{\mathrm{r} y} v_{\mathrm{t} z} \xi^{4}+10 A^{4} v_{\mathrm{r} z} v_{\mathrm{t} z} \xi^{4}+10 B^{4} v_{\mathrm{r} z} v_{\mathrm{t} z} \xi^{4}+20 A^{2} B^{2} v_{\mathrm{r} z} v_{\mathrm{t} z} \xi^{4}+8 A^{2} D^{2} v_{\mathrm{r} z} v_{\mathrm{t} z} \xi^{4}$ $+8 B^{2} D^{2} v_{\mathrm{r} z} v_{\mathrm{t} z} \xi^{4}-12 A D^{3} v_{\mathrm{t} x} v_{\mathrm{t} z} \xi^{4}-26 A^{3} D v_{\mathrm{t} x} v_{\mathrm{t} z} \xi^{4}-26 A B^{2} D v_{\mathrm{t} x} v_{\mathrm{t} z} \xi^{4}+12 A^{3} C v_{\mathrm{t} x} v_{\mathrm{t} z} \xi^{4}+12 A B^{2} C v_{\mathrm{t} x} v_{\mathrm{t} z} \xi^{4}$ $+28 A D^{2} C v_{\mathrm{t} x} v_{\mathrm{t} z} \xi^{4}-12 B D^{3} v_{\mathrm{t} y} v_{\mathrm{t} z} \xi^{4}-26 B^{3} D v_{\mathrm{t} y} v_{\mathrm{t} z} \xi^{4}-26 A^{2} B D v_{\mathrm{t} y} v_{\mathrm{t} z} \xi^{4}+12 B^{3} C v_{\mathrm{t} y} v_{\mathrm{t} z} \xi^{4}+28 B D^{2} C v_{\mathrm{t} y} v_{\mathrm{t} z} \xi^{4}$ $+12 A^{2} B C v_{\mathrm{t} y} v_{\mathrm{t} z} \xi^{4}+6 B^{4} v_{\mathrm{r} x}^{2} \xi^{2}+6 D^{4} v_{\mathrm{r} x}^{2} \xi^{2}+6 A^{2} B^{2} v_{\mathrm{r} x}^{2} \xi^{2}+6 A^{2} D^{2} v_{\mathrm{r} x}^{2} \xi^{2}+12 B^{2} D^{2} v_{\mathrm{r} x}^{2} \xi^{2}+6 A^{4} v_{\mathrm{r} y}^{2} \xi^{2}+6 D^{4} v_{\mathrm{r} y}^{2} \xi^{2}$ $+6 A^{2} B^{2} v_{\mathrm{r} y}^{2} \xi^{2}+12 A^{2} D^{2} v_{\mathrm{r} y}^{2} \xi^{2}+6 B^{2} D^{2} v_{\mathrm{r} y}^{2} \xi^{2}+6 A^{4} v_{\mathrm{r} z}^{2} \xi^{2}+6 B^{4} v_{\mathrm{r} z}^{2} \xi^{2}+12 A^{2} B^{2} v_{\mathrm{r} z}^{2} \xi^{2}+6 A^{2} D^{2} v_{\mathrm{r} z}^{2} \xi^{2}+6 B^{2} D^{2} v_{\mathrm{r} z}^{2} \xi^{2}$ $+6 B^{4} v_{\mathrm{t} x}^{2} \xi^{2}+6 D^{4} v_{\mathrm{t} x}^{2} \xi^{2}+6 A^{2} B^{2} v_{\mathrm{t} x}^{2} \xi^{2}+6 A^{2} D^{2} v_{\mathrm{t} x}^{2} \xi^{2}+12 B^{2} D^{2} v_{\mathrm{t} x}^{2} \xi^{2}+6 A^{4} v_{\mathrm{t} y}^{2} \xi^{2}+6 D^{4} v_{\mathrm{t} y}^{2} \xi^{2}+6 A^{2} B^{2} v_{\mathrm{t} y}^{2} \xi^{2}$ $+12 A^{2} D^{2} v_{\mathrm{t} y}^{2} \xi^{2}+6 B^{2} D^{2} v_{\mathrm{t} y}^{2} \xi^{2}+6 A^{4} v_{\mathrm{t} z}^{2} \xi^{2}+6 B^{4} v_{\mathrm{t} z}^{2} \xi^{2}+12 A^{2} B^{2} v_{\mathrm{t} z}^{2} \xi^{2}+6 A^{2} D^{2} v_{\mathrm{t} z}^{2} \xi^{2}+6 B^{2} D^{2} v_{\mathrm{t} z}^{2} \xi^{2}-12 A B^{3} v_{\mathrm{r} x} v_{\mathrm{r} y} \xi^{2}$ $-12 A B D^{2} v_{\mathrm{r} x} v_{\mathrm{r} y} \xi^{2}-12 A^{3} B v_{\mathrm{r} x} v_{\mathrm{r} y} \xi^{2}-12 A D^{3} v_{\mathrm{r} x} v_{\mathrm{r} z} \xi^{2}-12 A^{3} D v_{\mathrm{r} x} v_{\mathrm{r} z} \xi^{2}-12 A B^{2} D v_{\mathrm{r} x} v_{\mathrm{r} z} \xi^{2}-12 B D^{3} v_{\mathrm{r} y} v_{\mathrm{r} z} \xi^{2}$ $-12 B^{3} D v_{\mathrm{r} y} v_{\mathrm{r} z} \xi^{2}-12 A^{2} B D v_{\mathrm{r} y} v_{\mathrm{r} z} \xi^{2}+4 B^{4} v_{\mathrm{r} x} v_{\mathrm{t} x} \xi^{2}+4 D^{4} v_{\mathrm{r} x} v_{\mathrm{t} x} \xi^{2}+4 A^{2} B^{2} v_{\mathrm{r} x} v_{\mathrm{t} x} \xi^{2}+4 A^{2} D^{2} v_{\mathrm{r} x} v_{\mathrm{t} x} \xi^{2}$ $+8 B^{2} D^{2} v_{\mathrm{r} x} v_{\mathrm{t} x} \xi^{2}-4 A B^{3} v_{\mathrm{r} y} v_{\mathrm{t} x} \xi^{2}-4 A B D^{2} v_{\mathrm{r} y} v_{\mathrm{t} x} \xi^{2}-4 A^{3} B v_{\mathrm{r} y} v_{\mathrm{t} x} \xi^{2}-4 A D^{3} v_{\mathrm{r} z} v_{\mathrm{t} x} \xi^{2}-4 A^{3} D v_{\mathrm{r} z} v_{\mathrm{t} x} \xi^{2}$ $-4 A B^{2} D v_{\mathrm{r} z} v_{\mathrm{t} x} \xi^{2}-4 A B^{3} v_{\mathrm{r} x} v_{\mathrm{t} y} \xi^{2}-4 A B D^{2} v_{\mathrm{r} x} v_{\mathrm{t} y} \xi^{2}-4 A^{3} B v_{\mathrm{r} x} v_{\mathrm{t} y} \xi^{2}+4 A^{4} v_{\mathrm{r} y} v_{\mathrm{t} y} \xi^{2}+4 D^{4} v_{\mathrm{r} y} v_{\mathrm{t} y} \xi^{2}+4 A^{2} B^{2} v_{\mathrm{r} y} v_{\mathrm{t} y} \xi^{2}$ $+8 A^{2} D^{2} v_{\mathrm{r} y} v_{\mathrm{t} y} \xi^{2}+4 B^{2} D^{2} v_{\mathrm{r} y} v_{\mathrm{t} y} \xi^{2}-4 B D^{3} v_{\mathrm{r} z} v_{\mathrm{t} y} \xi^{2}-4 B^{3} D v_{\mathrm{r} z} v_{\mathrm{t} y} \xi^{2}-4 A^{2} B D v_{\mathrm{r} z} v_{\mathrm{t} y} \xi^{2}-12 A B^{3} v_{\mathrm{t} x} v_{\mathrm{t} y} \xi^{2}$ $-12 A B D^{2} v_{\mathrm{t} x} v_{\mathrm{t} y} \xi^{2}-12 A^{3} B v_{\mathrm{t} x} v_{\mathrm{t} y} \xi^{2}+4 A D^{3} v_{\mathrm{r} x} v_{\mathrm{t} z} \xi^{2}+4 A^{3} D v_{\mathrm{r} x} v_{\mathrm{t} z} \xi^{2}+4 A B^{2} D v_{\mathrm{r} x} v_{\mathrm{t} z} \xi^{2}+4 B D^{3} v_{\mathrm{r} y} v_{\mathrm{t} z} \xi^{2}$ $+4 B^{3} D v_{\mathrm{r} y} v_{\mathrm{t} z} \xi^{2}+4 A^{2} B D v_{\mathrm{r} y} v_{\mathrm{t} z} \xi^{2}-4 A^{4} v_{\mathrm{r} z} v_{\mathrm{t} z} \xi^{2}-4 B^{4} v_{\mathrm{r} z} v_{\mathrm{t} z} \xi^{2}-8 A^{2} B^{2} v_{\mathrm{r} z} v_{\mathrm{t} z} \xi^{2}-4 A^{2} D^{2} v_{\mathrm{r} z} v_{\mathrm{t} z} \xi^{2}-4 B^{2} D^{2} v_{\mathrm{r} z} v_{\mathrm{t} z} \xi^{2}$ $\left.+12 A D^{3} v_{\mathrm{t} x} v_{\mathrm{t} z} \xi^{2}+12 A^{3} D v_{\mathrm{t} x} v_{\mathrm{t} z} \xi^{2}+12 A B^{2} D v_{\mathrm{t} x} v_{\mathrm{t} z} \xi^{2}+12 B D^{3} v_{\mathrm{t} y} v_{\mathrm{t} z} \xi^{2}+12 B^{3} D v_{\mathrm{t} y} v_{\mathrm{t} z} \xi^{2}+12 A^{2} B D v_{\mathrm{t} y} v_{\mathrm{t} z} \xi^{2}\right) \eta^{2}$ 
$+\left(-2 B^{4} v_{\mathrm{r} x}^{2} \xi^{9}-2 D C^{3} v_{\mathrm{r} x}^{2} \xi^{9}-2 A^{2} B^{2} v_{\mathrm{r} x}^{2} \xi^{9}-4 A^{2} C^{2} v_{\mathrm{r} x}^{2} \xi^{9}-2 B^{2} C^{2} v_{\mathrm{r} x}^{2} \xi^{9}-2 B^{2} D C v_{\mathrm{r} x}^{2} \xi^{9}-2 A^{4} v_{\mathrm{r} y}^{2} \xi^{9}-2 D C^{3} v_{\mathrm{r} y}^{2} \xi^{9}\right.$ $-2 A^{2} B^{2} v_{\mathrm{r} y}^{2} \xi^{9}-2 A^{2} C^{2} v_{\mathrm{r} y}^{2} \xi^{9}-4 B^{2} C^{2} v_{\mathrm{r} y}^{2} \xi^{9}-2 A^{2} D C v_{\mathrm{r} y}^{2} \xi^{9}-4 A^{4} v_{\mathrm{r} z}^{2} \xi^{9}-4 B^{4} v_{\mathrm{r} z}^{2} \xi^{9}-8 A^{2} B^{2} v_{\mathrm{r} z}^{2} \xi^{9}-2 A^{2} D C v_{\mathrm{r} z}^{2} \xi^{9}$ $-2 B^{2} D C v_{\mathrm{r} z}^{2} \xi^{9}+2 B^{4} v_{\mathrm{t} x}^{2} \xi^{9}-2 D C^{3} v_{\mathrm{t} x}^{2} \xi^{9}+2 A^{2} B^{2} v_{\mathrm{t} x}^{2} \xi^{9}+4 A^{2} C^{2} v_{\mathrm{t} x}^{2} \xi^{9}+2 B^{2} C^{2} v_{\mathrm{t} x}^{2} \xi^{9}-2 B^{2} D C v_{\mathrm{t} x}^{2} \xi^{9}+2 A^{4} v_{\mathrm{t} y}^{2} \xi^{9}$ $-2 D C^{3} v_{\mathrm{t} y}^{2} \xi^{9}+2 A^{2} B^{2} v_{\mathrm{t} y}^{2} \xi^{9}+2 A^{2} C^{2} v_{\mathrm{t} y}^{2} \xi^{9}+4 B^{2} C^{2} v_{\mathrm{t} y}^{2} \xi^{9}-2 A^{2} D C v_{\mathrm{t} y}^{2} \xi^{9}+4 A^{4} v_{\mathrm{t} z}^{2} \xi^{9}+4 B^{4} v_{\mathrm{t} z}^{2} \xi^{9}+8 A^{2} B^{2} v_{\mathrm{t} z}^{2} \xi^{9}$ $-2 A^{2} D C v_{\mathrm{t} z}^{2} \xi^{9}-2 B^{2} D C v_{\mathrm{t} z}^{2} \xi^{9}+4 A B^{3} v_{\mathrm{r} x} v_{\mathrm{r} y} \xi^{9}-4 A B C^{2} v_{\mathrm{r} x} v_{\mathrm{r} y} \xi^{9}+4 A^{3} B v_{\mathrm{r} x} v_{\mathrm{r} y} \xi^{9}+4 A B D C v_{\mathrm{r} x} v_{\mathrm{r} y} \xi^{9}+4 A D C^{2} v_{\mathrm{r} x} v_{\mathrm{r} z} \xi^{9}$ $+8 A^{3} C v_{\mathrm{r} x} v_{\mathrm{r} z} \xi^{9}+8 A B^{2} C v_{\mathrm{r} x} v_{\mathrm{r} z} \xi^{9}+4 B D C^{2} v_{\mathrm{r} y} v_{\mathrm{r} z} \xi^{9}+8 B^{3} C v_{\mathrm{r} y} v_{\mathrm{r} z} \xi^{9}+8 A^{2} B C v_{\mathrm{r} y} v_{\mathrm{r} z} \xi^{9}-4 D C^{3} v_{\mathrm{r} x} v_{\mathrm{t} x} \xi^{9}$ $-4 B^{2} D C v_{\mathrm{r} x} v_{\mathrm{t} x} \xi^{9}+4 A B D C v_{\mathrm{r} y} v_{\mathrm{t} x} \xi^{9}+4 A D C^{2} v_{\mathrm{r} z} v_{\mathrm{t} x} \xi^{9}+4 A B D C v_{\mathrm{r} x} v_{\mathrm{t} y} \xi^{9}-4 D C^{3} v_{\mathrm{r} y} v_{\mathrm{t} y} \xi^{9}-4 A^{2} D C v_{\mathrm{r} y} v_{\mathrm{t} y} \xi^{9}$ $+4 B D C^{2} v_{\mathrm{r} z} v_{\mathrm{t} y} \xi^{9}-4 A B^{3} v_{\mathrm{t} x} v_{\mathrm{t} y} \xi^{9}+4 A B C^{2} v_{\mathrm{t} x} v_{\mathrm{t} y} \xi^{9}-4 A^{3} B v_{\mathrm{t} x} v_{\mathrm{t} y} \xi^{9}+4 A B D C v_{\mathrm{t} x} v_{\mathrm{t} y} \xi^{9}+4 A D C^{2} v_{\mathrm{r} x} v_{\mathrm{t} z} \xi^{9}$ $+4 B D C^{2} v_{\mathrm{r} y} v_{\mathrm{t} z} \xi^{9}-4 A^{2} D C v_{\mathrm{r} z} v_{\mathrm{t} z} \xi^{9}-4 B^{2} D C v_{\mathrm{r} z} v_{\mathrm{t} z} \xi^{9}+4 A D C^{2} v_{\mathrm{t} x} v_{\mathrm{t} z} \xi^{9}-8 A^{3} C v_{\mathrm{t} x} v_{\mathrm{t} z} \xi^{9}-8 A B^{2} C v_{\mathrm{t} x} v_{\mathrm{t} z} \xi^{9}$ $+4 B D C^{2} v_{\mathrm{t} y} v_{\mathrm{t} z} \xi^{9}-8 B^{3} C v_{\mathrm{t} y} v_{\mathrm{t} z} \xi^{9}-8 A^{2} B C v_{\mathrm{t} y} v_{\mathrm{t} z} \xi^{9}+8 B^{4} v_{\mathrm{r} x}^{2} \xi^{7}+8 A^{2} B^{2} v_{\mathrm{r} x}^{2} \xi^{7}+2 B^{2} D^{2} v_{\mathrm{r} x}^{2} \xi^{7}+4 A^{2} C^{2} v_{\mathrm{r} x}^{2} \xi^{7}$ $+2 B^{2} C^{2} v_{\mathrm{r} x}^{2} \xi^{7}+8 D^{2} C^{2} v_{\mathrm{r} x}^{2} \xi^{7}+8 A^{2} D C v_{\mathrm{r} x}^{2} \xi^{7}+12 B^{2} D C v_{\mathrm{r} x}^{2} \xi^{7}+8 A^{4} v_{\mathrm{r} y}^{2} \xi^{7}+8 A^{2} B^{2} v_{\mathrm{r} y}^{2} \xi^{7}+2 A^{2} D^{2} v_{\mathrm{r} y}^{2} \xi^{7}+2 A^{2} C^{2} v_{\mathrm{r} y}^{2} \xi^{7}$ $+4 B^{2} C^{2} v_{\mathrm{r} y}^{2} \xi^{7}+8 D^{2} C^{2} v_{\mathrm{r} y}^{2} \xi^{7}+12 A^{2} D C v_{\mathrm{r} y}^{2} \xi^{7}+8 B^{2} D C v_{\mathrm{r} y}^{2} \xi^{7}+12 A^{4} v_{\mathrm{r} z}^{2} \xi^{7}+12 B^{4} v_{\mathrm{r} z}^{2} \xi^{7}+24 A^{2} B^{2} v_{\mathrm{r} z}^{2} \xi^{7}+4 A^{2} D^{2} v_{\mathrm{r} z}^{2} \xi^{7}$ $+4 B^{2} D^{2} v_{\mathrm{r} z}^{2} \xi^{7}+4 A^{2} D C v_{\mathrm{r} z}^{2} \xi^{7}+4 B^{2} D C v_{\mathrm{r} z}^{2} \xi^{7}-8 B^{4} v_{\mathrm{t} x}^{2} \xi^{7}-8 A^{2} B^{2} v_{\mathrm{t} x}^{2} \xi^{7}-2 B^{2} D^{2} v_{\mathrm{t} x}^{2} \xi^{7}-4 A^{2} C^{2} v_{\mathrm{t} x}^{2} \xi^{7}-2 B^{2} C^{2} v_{\mathrm{t} x}^{2} \xi^{7}$ $-8 D^{2} C^{2} v_{\mathrm{t} x}^{2} \xi^{7}+8 A^{2} D C v_{\mathrm{t} x}^{2} \xi^{7}+12 B^{2} D C v_{\mathrm{t} x}^{2} \xi^{7}-8 A^{4} v_{\mathrm{t} y}^{2} \xi^{7}-8 A^{2} B^{2} v_{\mathrm{t} y}^{2} \xi^{7}-2 A^{2} D^{2} v_{\mathrm{t} y}^{2} \xi^{7}-2 A^{2} C^{2} v_{\mathrm{t} y}^{2} \xi^{7}-4 B^{2} C^{2} v_{\mathrm{t} y}^{2} \xi^{7}$ $-8 D^{2} C^{2} v_{\mathrm{t} y}^{2} \xi^{7}+12 A^{2} D C v_{\mathrm{t} y}^{2} \xi^{7}+8 B^{2} D C v_{\mathrm{t} y}^{2} \xi^{7}-12 A^{4} v_{\mathrm{t} z}^{2} \xi^{7}-12 B^{4} v_{\mathrm{t} z}^{2} \xi^{7}-24 A^{2} B^{2} v_{\mathrm{t} z}^{2} \xi^{7}-4 A^{2} D^{2} v_{\mathrm{t} z}^{2} \xi^{7}-4 B^{2} D^{2} v_{\mathrm{t} z}^{2} \xi^{7}$ $+4 A^{2} D C v_{\mathrm{t} z}^{2} \xi^{7}+4 B^{2} D C v_{\mathrm{t} z}^{2} \xi^{7}-16 A B^{3} v_{\mathrm{r} x} v_{\mathrm{r} y} \xi^{7}-4 A B D^{2} v_{\mathrm{r} x} v_{\mathrm{r} y} \xi^{7}+4 A B C^{2} v_{\mathrm{r} x} v_{\mathrm{r} y} \xi^{7}-16 A^{3} B v_{\mathrm{r} x} v_{\mathrm{r} y} \xi^{7}$ $-8 A B D C v_{\mathrm{r} x} v_{\mathrm{r} y} \xi^{7}-4 A D C^{2} v_{\mathrm{r} x} v_{\mathrm{r} z} \xi^{7}-8 A^{3} D v_{\mathrm{r} x} v_{\mathrm{r} z} \xi^{7}-8 A B^{2} D v_{\mathrm{r} x} v_{\mathrm{r} z} \xi^{7}-16 A^{3} C v_{\mathrm{r} x} v_{\mathrm{r} z} \xi^{7}-16 A B^{2} C v_{\mathrm{r} x} v_{\mathrm{r} z} \xi^{7}$ $-12 A D^{2} C v_{\mathrm{r} x} v_{\mathrm{r} z} \xi^{7}-4 B D C^{2} v_{\mathrm{r} y} v_{\mathrm{r} z} \xi^{7}-8 B^{3} D v_{\mathrm{r} y} v_{\mathrm{r} z} \xi^{7}-8 A^{2} B D v_{\mathrm{r} y} v_{\mathrm{r} z} \xi^{7}-16 B^{3} C v_{\mathrm{r} y} v_{\mathrm{r} z} \xi^{7}-12 B D^{2} C v_{\mathrm{r} y} v_{\mathrm{r} z} \xi^{7}$ $-16 A^{2} B C v_{\mathrm{r} y} v_{\mathrm{r} z} \xi^{7}-4 A D C^{2} v_{\mathrm{r} z} v_{\mathrm{t} x} \xi^{7}+4 A D^{2} C v_{\mathrm{r} z} v_{\mathrm{t} x} \xi^{7}-4 B D C^{2} v_{\mathrm{r} z} v_{\mathrm{t} y} \xi^{7}+4 B D^{2} C v_{\mathrm{r} z} v_{\mathrm{t} y} \xi^{7}+16 A B^{3} v_{\mathrm{t} x} v_{\mathrm{t} y} \xi^{7}$ $+4 A B D^{2} v_{\mathrm{t} x} v_{\mathrm{t} y} \xi^{7}-4 A B C^{2} v_{\mathrm{t} x} v_{\mathrm{t} y} \xi^{7}+16 A^{3} B v_{\mathrm{t} x} v_{\mathrm{t} y} \xi^{7}-8 A B D C v_{\mathrm{t} x} v_{\mathrm{t} y} \xi^{7}-4 A D C^{2} v_{\mathrm{r} x} v_{\mathrm{t} z} \xi^{7}-4 A D^{2} C v_{\mathrm{r} x} v_{\mathrm{t} z} \xi^{7}$ $-4 B D C^{2} v_{\mathrm{r} y} v_{\mathrm{t} z} \xi^{7}-4 B D^{2} C v_{\mathrm{r} y} v_{\mathrm{t} z} \xi^{7}+8 A^{2} D C v_{\mathrm{r} z} v_{\mathrm{t} z} \xi^{7}+8 B^{2} D C v_{\mathrm{r} z} v_{\mathrm{t} z} \xi^{7}-4 A D C^{2} v_{\mathrm{t} x} v_{\mathrm{t} z} \xi^{7}-8 A^{3} D v_{\mathrm{t} x} v_{\mathrm{t} z} \xi^{7}$ $-8 A B^{2} D v_{\mathrm{t} x} v_{\mathrm{t} z} \xi^{7}+16 A^{3} C v_{\mathrm{t} x} v_{\mathrm{t} z} \xi^{7}+16 A B^{2} C v_{\mathrm{t} x} v_{\mathrm{t} z} \xi^{7}+12 A D^{2} C v_{\mathrm{t} x} v_{\mathrm{t} z} \xi^{7}-4 B D C^{2} v_{\mathrm{t} y} v_{\mathrm{t} z} \xi^{7}-8 B^{3} D v_{\mathrm{t} y} v_{\mathrm{t} z} \xi^{7}$ $-8 A^{2} B D v_{\mathrm{t} y} v_{\mathrm{t} z} \xi^{7}+16 B^{3} C v_{\mathrm{t} y} v_{\mathrm{t} z} \xi^{7}+12 B D^{2} C v_{\mathrm{t} y} v_{\mathrm{t} z} \xi^{7}+16 A^{2} B C v_{\mathrm{t} y} v_{\mathrm{t} z} \xi^{7}-10 B^{4} v_{\mathrm{r} x}^{2} \xi^{5}-10 A^{2} B^{2} v_{\mathrm{r} x}^{2} \xi^{5}-4 A^{2} D^{2} v_{\mathrm{r} x}^{2} \xi^{5}$ $-10 B^{2} D^{2} v_{\mathrm{r} x}^{2} \xi^{5}-10 D^{3} C v_{\mathrm{r} x}^{2} \xi^{5}-8 A^{2} D C v_{\mathrm{r} x}^{2} \xi^{5}-10 B^{2} D C v_{\mathrm{r} x}^{2} \xi^{5}-10 A^{4} v_{\mathrm{r} y}^{2} \xi^{5}-10 A^{2} B^{2} v_{\mathrm{r} y}^{2} \xi^{5}-10 A^{2} D^{2} v_{\mathrm{r} y}^{2} \xi^{5}$ $-4 B^{2} D^{2} v_{\mathrm{r} y}^{2} \xi^{5}-10 D^{3} C v_{\mathrm{r} y}^{2} \xi^{5}-10 A^{2} D C v_{\mathrm{r} y}^{2} \xi^{5}-8 B^{2} D C v_{\mathrm{r} y}^{2} \xi^{5}-12 A^{4} v_{\mathrm{r} z}^{2} \xi^{5}-12 B^{4} v_{\mathrm{r} z}^{2} \xi^{5}-24 A^{2} B^{2} v_{\mathrm{r} z}^{2} \xi^{5}-8 A^{2} D^{2} v_{\mathrm{r} z}^{2} \xi^{5}$ $-8 B^{2} D^{2} v_{\mathrm{r} z}^{2} \xi^{5}-2 A^{2} D C v_{\mathrm{r} z}^{2} \xi^{5}-2 B^{2} D C v_{\mathrm{r} z}^{2} \xi^{5}+10 B^{4} v_{\mathrm{t} x}^{2} \xi^{5}+10 A^{2} B^{2} v_{\mathrm{t} x}^{2} \xi^{5}+4 A^{2} D^{2} v_{\mathrm{tx}}^{2} \xi^{5}+10 B^{2} D^{2} v_{\mathrm{t} x}^{2} \xi^{5}$ $-10 D^{3} C v_{\mathrm{t} x}^{2} \xi^{5}-8 A^{2} D C v_{\mathrm{t} x}^{2} \xi^{5}-10 B^{2} D C v_{\mathrm{t} x}^{2} \xi^{5}+10 A^{4} v_{\mathrm{t} y}^{2} \xi^{5}+10 A^{2} B^{2} v_{\mathrm{t} y}^{2} \xi^{5}+10 A^{2} D^{2} v_{\mathrm{t} y}^{2} \xi^{5}+4 B^{2} D^{2} v_{\mathrm{t} y}^{2} \xi^{5}$ $-10 D^{3} C v_{\mathrm{t} y}^{2} \xi^{5}-10 A^{2} D C v_{\mathrm{t} y}^{2} \xi^{5}-8 B^{2} D C v_{\mathrm{t} y}^{2} \xi^{5}+12 A^{4} v_{\mathrm{t} z}^{2} \xi^{5}+12 B^{4} v_{\mathrm{t} z}^{2} \xi^{5}+24 A^{2} B^{2} v_{\mathrm{t} z}^{2} \xi^{5}+8 A^{2} D^{2} v_{\mathrm{t} z}^{2} \xi^{5}+8 B^{2} D^{2} v_{\mathrm{t} z}^{2} \xi^{5}$ $-2 A^{2} D C v_{\mathrm{t} z}^{2} \xi^{5}-2 B^{2} D C v_{\mathrm{t} z}^{2} \xi^{5}+20 A B^{3} v_{\mathrm{r} x} v_{\mathrm{r} y} \xi^{5}+12 A B D^{2} v_{\mathrm{r} x} v_{\mathrm{r} y} \xi^{5}+20 A^{3} B v_{\mathrm{r} x} v_{\mathrm{r} y} \xi^{5}+4 A B D C v_{\mathrm{r} x} v_{\mathrm{r} y} \xi^{5}$ $+8 A D^{3} v_{\mathrm{r} x} v_{\mathrm{r} z} \xi^{5}+16 A^{3} D v_{\mathrm{r} x} v_{\mathrm{r} z} \xi^{5}+16 A B^{2} D v_{\mathrm{r} x} v_{\mathrm{r} z} \xi^{5}+8 A^{3} C v_{\mathrm{r} x} v_{\mathrm{r} z} \xi^{5}+8 A B^{2} C v_{\mathrm{r} x} v_{\mathrm{r} z} \xi^{5}+12 A D^{2} C v_{\mathrm{r} x} v_{\mathrm{r} z} \xi^{5}$ $+8 B D^{3} v_{\mathrm{r} y} v_{\mathrm{r} z} \xi^{5}+16 B^{3} D v_{\mathrm{r} y} v_{\mathrm{r} z} \xi^{5}+16 A^{2} B D v_{\mathrm{r} y} v_{\mathrm{r} z} \xi^{5}+8 B^{3} C v_{\mathrm{r} y} v_{\mathrm{r} z} \xi^{5}+12 B D^{2} C v_{\mathrm{r} y} v_{\mathrm{r} z} \xi^{5}+8 A^{2} B C v_{\mathrm{r} y} v_{\mathrm{r} z} \xi^{5}$ $+4 D^{3} C v_{\mathrm{r} x} v_{\mathrm{t} x} \xi^{5}+4 B^{2} D C v_{\mathrm{r} x} v_{\mathrm{t} x} \xi^{5}-4 A B D C v_{\mathrm{r} y} v_{\mathrm{t} x} \xi^{5}-4 A D^{2} C v_{\mathrm{r} z} v_{\mathrm{t} x} \xi^{5}-4 A B D C v_{\mathrm{r} x} v_{\mathrm{t} y} \xi^{5}+4 D^{3} C v_{\mathrm{r} y} v_{\mathrm{t} y} \xi^{5}$ $+4 A^{2} D C v_{\mathrm{r} y} v_{\mathrm{t} y} \xi^{5}-4 B D^{2} C v_{\mathrm{r} z} v_{\mathrm{t} y} \xi^{5}-20 A B^{3} v_{\mathrm{t} x} v_{\mathrm{t} y} \xi^{5}-12 A B D^{2} v_{\mathrm{t} x} v_{\mathrm{t} y} \xi^{5}-20 A^{3} B v_{\mathrm{t} x} v_{\mathrm{t} y} \xi^{5}+4 A B D C v_{\mathrm{t} x} v_{\mathrm{t} y} \xi^{5}$ $+4 A D^{2} C v_{\mathrm{r} x} v_{\mathrm{t} z} \xi^{5}+4 B D^{2} C v_{\mathrm{r} y} v_{\mathrm{t} z} \xi^{5}-4 A^{2} D C v_{\mathrm{r} z} v_{\mathrm{t} z} \xi^{5}-4 B^{2} D C v_{\mathrm{r} z} v_{\mathrm{t} z} \xi^{5}+8 A D^{3} v_{\mathrm{t} x} v_{\mathrm{t} z} \xi^{5}+16 A^{3} D v_{\mathrm{t} x} v_{\mathrm{t} z} \xi^{5}$ $+16 A B^{2} D v_{\mathrm{t} x} v_{\mathrm{t} z} \xi^{5}-8 A^{3} C v_{\mathrm{t} x} v_{\mathrm{t} z} \xi^{5}-8 A B^{2} C v_{\mathrm{t} x} v_{\mathrm{t} z} \xi^{5}-12 A D^{2} C v_{\mathrm{t} x} v_{\mathrm{t} z} \xi^{5}+8 B D^{3} v_{\mathrm{t} y} v_{\mathrm{t} z} \xi^{5}+16 B^{3} D v_{\mathrm{t} y} v_{\mathrm{t} z} \xi^{5}$ $+16 A^{2} B D v_{\mathrm{t} y} v_{\mathrm{t} z} \xi^{5}-8 B^{3} C v_{\mathrm{t} y} v_{\mathrm{t} z} \xi^{5}-12 B D^{2} C v_{\mathrm{t} y} v_{\mathrm{t} z} \xi^{5}-8 A^{2} B C v_{\mathrm{t} y} v_{\mathrm{t} z} \xi^{5}+4 B^{4} v_{\mathrm{r} x}^{2} \xi^{3}+4 D^{4} v_{\mathrm{r} x}^{2} \xi^{3}+4 A^{2} B^{2} v_{\mathrm{r} x}^{2} \xi^{3}$ $+4 A^{2} D^{2} v_{\mathrm{r} x}^{2} \xi^{3}+8 B^{2} D^{2} v_{\mathrm{r} x}^{2} \xi^{3}+4 A^{4} v_{\mathrm{r} y}^{2} \xi^{3}+4 D^{4} v_{\mathrm{r} y}^{2} \xi^{3}+4 A^{2} B^{2} v_{\mathrm{r} y}^{2} \xi^{3}+8 A^{2} D^{2} v_{\mathrm{r} y}^{2} \xi^{3}+4 B^{2} D^{2} v_{\mathrm{r} y}^{2} \xi^{3}+4 A^{4} v_{\mathrm{r} z}^{2} \xi^{3}$ $+4 B^{4} v_{\mathrm{r} z}^{2} \xi^{3}+8 A^{2} B^{2} v_{\mathrm{r} z}^{2} \xi^{3}+4 A^{2} D^{2} v_{\mathrm{r} z}^{2} \xi^{3}+4 B^{2} D^{2} v_{\mathrm{r} z}^{2} \xi^{3}-4 B^{4} v_{\mathrm{t} x}^{2} \xi^{3}-4 D^{4} v_{\mathrm{t} x}^{2} \xi^{3}-4 A^{2} B^{2} v_{\mathrm{t} x}^{2} \xi^{3}-4 A^{2} D^{2} v_{\mathrm{t} x}^{2} \xi^{3}$ $-8 B^{2} D^{2} v_{\mathrm{t} x}^{2} \xi^{3}-4 A^{4} v_{\mathrm{t} y}^{2} \xi^{3}-4 D^{4} v_{\mathrm{t} y}^{2} \xi^{3}-4 A^{2} B^{2} v_{\mathrm{t} y}^{2} \xi^{3}-8 A^{2} D^{2} v_{\mathrm{t} y}^{2} \xi^{3}-4 B^{2} D^{2} v_{\mathrm{t} y}^{2} \xi^{3}-4 A^{4} v_{\mathrm{t} z}^{2} \xi^{3}-4 B^{4} v_{\mathrm{t} z}^{2} \xi^{3}$ $-8 A^{2} B^{2} v_{\mathrm{t} z}^{2} \xi^{3}-4 A^{2} D^{2} v_{\mathrm{t} z}^{2} \xi^{3}-4 B^{2} D^{2} v_{\mathrm{t} z}^{2} \xi^{3}-8 A B^{3} v_{\mathrm{r} x} v_{\mathrm{r} y} \xi^{3}-8 A B D^{2} v_{\mathrm{r} x} v_{\mathrm{r} y} \xi^{3}-8 A^{3} B v_{\mathrm{r} x} v_{\mathrm{r} y} \xi^{3}-8 A D^{3} v_{\mathrm{r} x} v_{\mathrm{r} z} \xi^{3}$ $-8 A^{3} D v_{\mathrm{r} x} v_{\mathrm{r} z} \xi^{3}-8 A B^{2} D v_{\mathrm{r} x} v_{\mathrm{r} z} \xi^{3}-8 B D^{3} v_{\mathrm{r} y} v_{\mathrm{r} z} \xi^{3}-8 B^{3} D v_{\mathrm{r} y} v_{\mathrm{r} z} \xi^{3}-8 A^{2} B D v_{\mathrm{r} y} v_{\mathrm{r} z} \xi^{3}+8 A B^{3} v_{\mathrm{t} x} v_{\mathrm{t} y} \xi^{3}$ $+8 A B D^{2} v_{\mathrm{t} x} v_{\mathrm{t} y} \xi^{3}+8 A^{3} B v_{\mathrm{t} x} v_{\mathrm{t} y} \xi^{3}-8 A D^{3} v_{\mathrm{t} x} v_{\mathrm{t} z} \xi^{3}-8 A^{3} D v_{\mathrm{t} x} v_{\mathrm{t} z} \xi^{3}-8 A B^{2} D v_{\mathrm{t} x} v_{\mathrm{t} z} \xi^{3}-8 B D^{3} v_{\mathrm{t} y} v_{\mathrm{t} z} \xi^{3}$ $\left.-8 B^{3} D v_{\mathrm{t} y} v_{\mathrm{t} z} \xi^{3}-8 A^{2} B D v_{\mathrm{t} y} v_{\mathrm{t} z} \xi^{3}\right) \eta$ 
$-A^{2} C^{2} v_{\mathrm{r} x}^{2} \xi^{10}-B^{2} C^{2} v_{\mathrm{r} y}^{2} \xi^{10}-A^{4} v_{\mathrm{r} z}^{2} \xi^{10}-B^{4} v_{\mathrm{r} z}^{2} \xi^{10}-2 A^{2} B^{2} v_{\mathrm{r} z}^{2} \xi^{10}-A^{2} C^{2} v_{\mathrm{t} x}^{2} \xi^{10}-B^{2} C^{2} v_{\mathrm{t} y}^{2} \xi^{10}-A^{4} v_{\mathrm{t} z}^{2} \xi^{10}$ $-B^{4} v_{\mathrm{t} z}^{2} \xi^{10}-2 A^{2} B^{2} v_{\mathrm{t} z}^{2} \xi^{10}-2 A B C^{2} v_{\mathrm{r} x} v_{\mathrm{r} y} \xi^{10}+2 A^{3} C v_{\mathrm{r} x} v_{\mathrm{r} z} \xi^{10}+2 A B^{2} C v_{\mathrm{r} x} v_{\mathrm{r} z} \xi^{10}+2 B^{3} C v_{\mathrm{r} y} v_{\mathrm{r} z} \xi^{10}$ $+2 A^{2} B C v_{\mathrm{r} y} v_{\mathrm{r} z} \xi^{10}-2 A^{2} C^{2} v_{\mathrm{r} x} v_{\mathrm{t} x} \xi^{10}-2 A B C^{2} v_{\mathrm{r} y} v_{\mathrm{t} x} \xi^{10}+2 A^{3} C v_{\mathrm{r} z} v_{\mathrm{t} x} \xi^{10}+2 A B^{2} C v_{\mathrm{r} z} v_{\mathrm{t} x} \xi^{10}-2 A B C^{2} v_{\mathrm{r} x} v_{\mathrm{t} y} \xi^{10}$ $-2 B^{2} C^{2} v_{\mathrm{r} y} v_{\mathrm{t} y} \xi^{10}+2 B^{3} C v_{\mathrm{r} z} v_{\mathrm{t} y} \xi^{10}+2 A^{2} B C v_{\mathrm{r} z} v_{\mathrm{t} y} \xi^{10}-2 A B C^{2} v_{\mathrm{t} x} v_{\mathrm{t} y} \xi^{10}+2 A^{3} C v_{\mathrm{r} x} v_{\mathrm{t} z} \xi^{10}+2 A B^{2} C v_{\mathrm{r} x} v_{\mathrm{t} z} \xi^{10}$ $+2 B^{3} C v_{\mathrm{r} y} v_{\mathrm{t} z} \xi^{10}+2 A^{2} B C v_{\mathrm{r} y} v_{\mathrm{t} z} \xi^{10}-2 A^{4} v_{\mathrm{r} z} v_{\mathrm{t} z} \xi^{10}-2 B^{4} v_{\mathrm{r} z} v_{\mathrm{t} z} \xi^{10}-4 A^{2} B^{2} v_{\mathrm{r} z} v_{\mathrm{t} z} \xi^{10}+2 A^{3} C v_{\mathrm{t} x} v_{\mathrm{t} z} \xi^{10}$ $+2 A B^{2} C v_{\mathrm{t} x} v_{\mathrm{t} z} \xi^{10}+2 B^{3} C v_{\mathrm{t} y} v_{\mathrm{t} z} \xi^{10}+2 A^{2} B C v_{\mathrm{t} y} v_{\mathrm{t} z} \xi^{10}+B^{4} v_{\mathrm{r} x}^{2} \xi^{8}+A^{2} B^{2} v_{\mathrm{r} x}^{2} \xi^{8}+A^{2} C^{2} v_{\mathrm{r} x}^{2} \xi^{8}+D^{2} C^{2} v_{\mathrm{r} x}^{2} \xi^{8}$ $+2 A^{2} D C v_{\mathrm{r} x}^{2} \xi^{8}+2 B^{2} D C v_{\mathrm{r} x}^{2} \xi^{8}+A^{4} v_{\mathrm{r} y}^{2} \xi^{8}+A^{2} B^{2} v_{\mathrm{r} y}^{2} \xi^{8}+B^{2} C^{2} v_{\mathrm{r} y}^{2} \xi^{8}+D^{2} C^{2} v_{\mathrm{r} y}^{2} \xi^{8}+2 A^{2} D C v_{\mathrm{r} y}^{2} \xi^{8}+2 B^{2} D C v_{\mathrm{r} y}^{2} \xi^{8}$ $+3 A^{4} v_{\mathrm{r} z}^{2} \xi^{8}+3 B^{4} v_{\mathrm{r} z}^{2} \xi^{8}+6 A^{2} B^{2} v_{\mathrm{r} z}^{2} \xi^{8}+A^{2} D^{2} v_{\mathrm{r} z}^{2} \xi^{8}+B^{2} D^{2} v_{\mathrm{r} z}^{2} \xi^{8}+B^{4} v_{\mathrm{t} x}^{2} \xi^{8}+A^{2} B^{2} v_{\mathrm{t} x}^{2} \xi^{8}+A^{2} C^{2} v_{\mathrm{t} x}^{2} \xi^{8}+D^{2} C^{2} v_{\mathrm{t} x}^{2} \xi^{8}$ $-2 A^{2} D C v_{\mathrm{t} x}^{2} \xi^{8}-2 B^{2} D C v_{\mathrm{t} x}^{2} \xi^{8}+A^{4} v_{\mathrm{t} y}^{2} \xi^{8}+A^{2} B^{2} v_{\mathrm{t} y}^{2} \xi^{8}+B^{2} C^{2} v_{\mathrm{t} y}^{2} \xi^{8}+D^{2} C^{2} v_{\mathrm{t} y}^{2} \xi^{8}-2 A^{2} D C v_{\mathrm{t} y}^{2} \xi^{8}-2 B^{2} D C v_{\mathrm{t} y}^{2} \xi^{8}$ $+3 A^{4} v_{\mathrm{t} z}^{2} \xi^{8}+3 B^{4} v_{\mathrm{t} z}^{2} \xi^{8}+6 A^{2} B^{2} v_{\mathrm{t} z}^{2} \xi^{8}+A^{2} D^{2} v_{\mathrm{t} z}^{2} \xi^{8}+B^{2} D^{2} v_{\mathrm{t} z}^{2} \xi^{8}-2 A B^{3} v_{\mathrm{r} x} v_{\mathrm{r} y} \xi^{8}+2 A B C^{2} v_{\mathrm{r} x} v_{\mathrm{r} y} \xi^{8}-2 A^{3} B v_{\mathrm{r} x} v_{\mathrm{r} y} \xi^{8}$ $-2 A^{3} D v_{\mathrm{r} x} v_{\mathrm{r} z} \xi^{8}-2 A B^{2} D v_{\mathrm{r} x} v_{\mathrm{r} z} \xi^{8}-4 A^{3} C v_{\mathrm{r} x} v_{\mathrm{r} z} \xi^{8}-4 A B^{2} C v_{\mathrm{r} x} v_{\mathrm{r} z} \xi^{8}-2 A D^{2} C v_{\mathrm{r} x} v_{\mathrm{r} z} \xi^{8}-2 B^{3} D v_{\mathrm{r} y} v_{\mathrm{r} z} \xi^{8}$ $-2 A^{2} B D v_{\mathrm{r} y} v_{\mathrm{r} z} \xi^{8}-4 B^{3} C v_{\mathrm{r} y} v_{\mathrm{r} z} \xi^{8}-2 B D^{2} C v_{\mathrm{r} y} v_{\mathrm{r} z} \xi^{8}-4 A^{2} B C v_{\mathrm{r} y} v_{\mathrm{r} z} \xi^{8}-2 B^{4} v_{\mathrm{r} x} v_{\mathrm{t} x} \xi^{8}-2 A^{2} B^{2} v_{\mathrm{r} x} v_{\mathrm{t} x} \xi^{8}$ $+2 A^{2} C^{2} v_{\mathrm{r} x} v_{\mathrm{t} x} \xi^{8}+2 D^{2} C^{2} v_{\mathrm{r} x} v_{\mathrm{t} x} \xi^{8}+2 A B^{3} v_{\mathrm{r} y} v_{\mathrm{t} x} \xi^{8}+2 A B C^{2} v_{\mathrm{r} y} v_{\mathrm{t} x} \xi^{8}+2 A^{3} B v_{\mathrm{r} y} v_{\mathrm{t} x} \xi^{8}+2 A^{3} D v_{\mathrm{r} z} v_{\mathrm{t} x} \xi^{8}$ $+2 A B^{2} D v_{\mathrm{r} z} v_{\mathrm{t} x} \xi^{8}-4 A^{3} C v_{\mathrm{r} z} v_{\mathrm{t} x} \xi^{8}-4 A B^{2} C v_{\mathrm{r} z} v_{\mathrm{t} x} \xi^{8}-2 A D^{2} C v_{\mathrm{r} z} v_{\mathrm{t} x} \xi^{8}+2 A B^{3} v_{\mathrm{r} x} v_{\mathrm{t} y} \xi^{8}+2 A B C^{2} v_{\mathrm{r} x} v_{\mathrm{t} y} \xi^{8}$ $+2 A^{3} B v_{\mathrm{r} x} v_{\mathrm{t} y} \xi^{8}-2 A^{4} v_{\mathrm{r} y} v_{\mathrm{t} y} \xi^{8}-2 A^{2} B^{2} v_{\mathrm{r} y} v_{\mathrm{t} y} \xi^{8}+2 B^{2} C^{2} v_{\mathrm{r} y} v_{\mathrm{t} y} \xi^{8}+2 D^{2} C^{2} v_{\mathrm{r} y} v_{\mathrm{t} y} \xi^{8}+2 B^{3} D v_{\mathrm{r} z} v_{\mathrm{t} y} \xi^{8}$ $+2 A^{2} B D v_{\mathrm{r} z} v_{\mathrm{t} y} \xi^{8}-4 B^{3} C v_{\mathrm{r} z} v_{\mathrm{t} y} \xi^{8}-2 B D^{2} C v_{\mathrm{r} z} v_{\mathrm{t} y} \xi^{8}-4 A^{2} B C v_{\mathrm{r} z} v_{\mathrm{t} y} \xi^{8}-2 A B^{3} v_{\mathrm{t} x} v_{\mathrm{t} y} \xi^{8}+2 A B C^{2} v_{\mathrm{t} x} v_{\mathrm{t} y} \xi^{8}$ $-2 A^{3} B v_{\mathrm{t} x} v_{\mathrm{t} y} \xi^{8}-2 A^{3} D v_{\mathrm{r} x} v_{\mathrm{t} z} \xi^{8}-2 A B^{2} D v_{\mathrm{r} x} v_{\mathrm{t} z} \xi^{8}-4 A^{3} C v_{\mathrm{r} x} v_{\mathrm{t} z} \xi^{8}-4 A B^{2} C v_{\mathrm{r} x} v_{\mathrm{t} z} \xi^{8}-2 A D^{2} C v_{\mathrm{r} x} v_{\mathrm{t} z} \xi^{8}$ $-2 B^{3} D v_{\mathrm{r} y} v_{\mathrm{t} z} \xi^{8}-2 A^{2} B D v_{\mathrm{r} y} v_{\mathrm{t} z} \xi^{8}-4 B^{3} C v_{\mathrm{r} y} v_{\mathrm{t} z} \xi^{8}-2 B D^{2} C v_{\mathrm{r} y} v_{\mathrm{t} z} \xi^{8}-4 A^{2} B C v_{\mathrm{r} y} v_{\mathrm{t} z} \xi^{8}+6 A^{4} v_{\mathrm{r} z} v_{\mathrm{t} z} \xi^{8}+6 B^{4} v_{\mathrm{r} z} v_{\mathrm{t} z} \xi^{8}$ $+12 A^{2} B^{2} v_{\mathrm{r} z} v_{\mathrm{t} z} \xi^{8}+2 A^{2} D^{2} v_{\mathrm{r} z} v_{\mathrm{t} z} \xi^{8}+2 B^{2} D^{2} v_{\mathrm{r} z} v_{\mathrm{t} z} \xi^{8}+2 A^{3} D v_{\mathrm{t} x} v_{\mathrm{t} z} \xi^{8}+2 A B^{2} D v_{\mathrm{t} x} v_{\mathrm{t} z} \xi^{8}-4 A^{3} C v_{\mathrm{t} x} v_{\mathrm{t} z} \xi^{8}$ $-4 A B^{2} C v_{\mathrm{t} x} v_{\mathrm{t} z} \xi^{8}-2 A D^{2} C v_{\mathrm{t} x} v_{\mathrm{t} z} \xi^{8}+2 B^{3} D v_{\mathrm{t} y} v_{\mathrm{t} z} \xi^{8}+2 A^{2} B D v_{\mathrm{t} y} v_{\mathrm{t} z} \xi^{8}-4 B^{3} C v_{\mathrm{t} y} v_{\mathrm{t} z} \xi^{8}-2 B D^{2} C v_{\mathrm{t} y} v_{\mathrm{t} z} \xi^{8}$ $-4 A^{2} B C v_{\mathrm{t} y} v_{\mathrm{t} z} \xi^{8}-2 B^{4} v_{\mathrm{r} x}^{2} \xi^{6}-2 A^{2} B^{2} v_{\mathrm{r} x}^{2} \xi^{6}-A^{2} D^{2} v_{\mathrm{r} x}^{2} \xi^{6}-2 B^{2} D^{2} v_{\mathrm{r} x}^{2} \xi^{6}-2 D^{3} C v_{\mathrm{r} x}^{2} \xi^{6}-2 A^{2} D C v_{\mathrm{r} x}^{2} \xi^{6}-2 B^{2} D C v_{\mathrm{r} x}^{2} \xi^{6}$ $-2 A^{4} v_{\mathrm{r} y}^{2} \xi^{6}-2 A^{2} B^{2} v_{\mathrm{r} y}^{2} \xi^{6}-2 A^{2} D^{2} v_{\mathrm{r} y}^{2} \xi^{6}-B^{2} D^{2} v_{\mathrm{r} y}^{2} \xi^{6}-2 D^{3} C v_{\mathrm{r} y}^{2} \xi^{6}-2 A^{2} D C v_{\mathrm{r} y}^{2} \xi^{6}-2 B^{2} D C v_{\mathrm{r} y}^{2} \xi^{6}-3 A^{4} v_{\mathrm{r} z}^{2} \xi^{6}$ $-3 B^{4} v_{\mathrm{r} z}^{2} \xi^{6}-6 A^{2} B^{2} v_{\mathrm{r} z}^{2} \xi^{6}-2 A^{2} D^{2} v_{\mathrm{r} z}^{2} \xi^{6}-2 B^{2} D^{2} v_{\mathrm{r} z}^{2} \xi^{6}-2 B^{4} v_{\mathrm{t} x}^{2} \xi^{6}-2 A^{2} B^{2} v_{\mathrm{t} x}^{2} \xi^{6}-A^{2} D^{2} v_{\mathrm{t} x}^{2} \xi^{6}-2 B^{2} D^{2} v_{\mathrm{t} x}^{2} \xi^{6}$ $+2 D^{3} C v_{\mathrm{t} x}^{2} \xi^{6}+2 A^{2} D C v_{\mathrm{t} x}^{2} \xi^{6}+2 B^{2} D C v_{\mathrm{t} x}^{2} \xi^{6}-2 A^{4} v_{\mathrm{t} y}^{2} \xi^{6}-2 A^{2} B^{2} v_{\mathrm{t} y}^{2} \xi^{6}-2 A^{2} D^{2} v_{\mathrm{t} y}^{2} \xi^{6}-B^{2} D^{2} v_{\mathrm{t} y}^{2} \xi^{6}+2 D^{3} C v_{\mathrm{t} y}^{2} \xi^{6}$ $+2 A^{2} D C v_{\mathrm{t} y}^{2} \xi^{6}+2 B^{2} D C v_{\mathrm{t} y}^{2} \xi^{6}-3 A^{4} v_{\mathrm{t} z}^{2} \xi^{6}-3 B^{4} v_{\mathrm{t} z}^{2} \xi^{6}-6 A^{2} B^{2} v_{\mathrm{t} z}^{2} \xi^{6}-2 A^{2} D^{2} v_{\mathrm{t} z}^{2} \xi^{6}-2 B^{2} D^{2} v_{\mathrm{t} z}^{2} \xi^{6}+4 A B^{3} v_{\mathrm{r} x} v_{\mathrm{r} y} \xi^{6}$ $+2 A B D^{2} v_{\mathrm{r} x} v_{\mathrm{r} y} \xi^{6}+4 A^{3} B v_{\mathrm{r} x} v_{\mathrm{r} y} \xi^{6}+2 A D^{3} v_{\mathrm{r} x} v_{\mathrm{r} z} \xi^{6}+4 A^{3} D v_{\mathrm{r} x} v_{\mathrm{r} z} \xi^{6}+4 A B^{2} D v_{\mathrm{r} x} v_{\mathrm{r} z} \xi^{6}+2 A^{3} C v_{\mathrm{r} x} v_{\mathrm{r} z} \xi^{6}$ $+2 A B^{2} C v_{\mathrm{r} x} v_{\mathrm{r} z} \xi^{6}+2 A D^{2} C v_{\mathrm{r} x} v_{\mathrm{r} z} \xi^{6}+2 B D^{3} v_{\mathrm{r} y} v_{\mathrm{r} z} \xi^{6}+4 B^{3} D v_{\mathrm{r} y} v_{\mathrm{r} z} \xi^{6}+4 A^{2} B D v_{\mathrm{r} y} v_{\mathrm{r} z} \xi^{6}+2 B^{3} C v_{\mathrm{r} y} v_{\mathrm{r} z} \xi^{6}$ $+2 B D^{2} C v_{\mathrm{r} y} v_{\mathrm{r} z} \xi^{6}+2 A^{2} B C v_{\mathrm{r} y} v_{\mathrm{r} z} \xi^{6}+4 B^{4} v_{\mathrm{r} x} v_{\mathrm{t} x} \xi^{6}+4 A^{2} B^{2} v_{\mathrm{r} x} v_{\mathrm{t} x} \xi^{6}+2 A^{2} D^{2} v_{\mathrm{r} x} v_{\mathrm{t} x} \xi^{6}+4 B^{2} D^{2} v_{\mathrm{r} x} v_{\mathrm{t} x} \xi^{6}$ $-4 A B^{3} v_{\mathrm{r} y} v_{\mathrm{t} x} \xi^{6}-2 A B D^{2} v_{\mathrm{r} y} v_{\mathrm{t} x} \xi^{6}-4 A^{3} B v_{\mathrm{r} y} v_{\mathrm{t} x} \xi^{6}-2 A D^{3} v_{\mathrm{r} z} v_{\mathrm{t} x} \xi^{6}-4 A^{3} D v_{\mathrm{r} z} v_{\mathrm{t} x} \xi^{6}-4 A B^{2} D v_{\mathrm{r} z} v_{\mathrm{t} x} \xi^{6}$ $+2 A^{3} C v_{\mathrm{r} z} v_{\mathrm{t} x} \xi^{6}+2 A B^{2} C v_{\mathrm{r} z} v_{\mathrm{t} x} \xi^{6}+2 A D^{2} C v_{\mathrm{r} z} v_{\mathrm{t} x} \xi^{6}-4 A B^{3} v_{\mathrm{r} x} v_{\mathrm{t} y} \xi^{6}-2 A B D^{2} v_{\mathrm{r} x} v_{\mathrm{t} y} \xi^{6}-4 A^{3} B v_{\mathrm{r} x} v_{\mathrm{t} y} \xi^{6}$ $+4 A^{4} v_{\mathrm{r} y} v_{\mathrm{t} y} \xi^{6}+4 A^{2} B^{2} v_{\mathrm{r} y} v_{\mathrm{t} y} \xi^{6}+4 A^{2} D^{2} v_{\mathrm{r} y} v_{\mathrm{t} y} \xi^{6}+2 B^{2} D^{2} v_{\mathrm{r} y} v_{\mathrm{t} y} \xi^{6}-2 B D^{3} v_{\mathrm{r} z} v_{\mathrm{t} y} \xi^{6}-4 B^{3} D v_{\mathrm{r} z} v_{\mathrm{t} y} \xi^{6}$ $-4 A^{2} B D v_{\mathrm{r} z} v_{\mathrm{t} y} \xi^{6}+2 B^{3} C v_{\mathrm{r} z} v_{\mathrm{t} y} \xi^{6}+2 B D^{2} C v_{\mathrm{r} z} v_{\mathrm{t} y} \xi^{6}+2 A^{2} B C v_{\mathrm{r} z} v_{\mathrm{t} y} \xi^{6}+4 A B^{3} v_{\mathrm{t} x} v_{\mathrm{t} y} \xi^{6}+2 A B D^{2} v_{\mathrm{t} x} v_{\mathrm{t} y} \xi^{6}$ $+4 A^{3} B v_{\mathrm{t} x} v_{\mathrm{t} y} \xi^{6}+2 A D^{3} v_{\mathrm{r} x} v_{\mathrm{t} z} \xi^{6}+4 A^{3} D v_{\mathrm{r} x} v_{\mathrm{t} z} \xi^{6}+4 A B^{2} D v_{\mathrm{r} x} v_{\mathrm{t} z} \xi^{6}+2 A^{3} C v_{\mathrm{r} x} v_{\mathrm{t} z} \xi^{6}+2 A B^{2} C v_{\mathrm{r} x} v_{\mathrm{t} z} \xi^{6}$ $+2 A D^{2} C v_{\mathrm{r} x} v_{\mathrm{t} z} \xi^{6}+2 B D^{3} v_{\mathrm{r} y} v_{\mathrm{t} z} \xi^{6}+4 B^{3} D v_{\mathrm{r} y} v_{\mathrm{t} z} \xi^{6}+4 A^{2} B D v_{\mathrm{r} y} v_{\mathrm{t} z} \xi^{6}+2 B^{3} C v_{\mathrm{r} y} v_{\mathrm{t} z} \xi^{6}+2 B D^{2} C v_{\mathrm{r} y} v_{\mathrm{t} z} \xi^{6}$ $+2 A^{2} B C v_{\mathrm{r} y} v_{\mathrm{t} z} \xi^{6}-6 A^{4} v_{\mathrm{r} z} v_{\mathrm{t} z} \xi^{6}-6 B^{4} v_{\mathrm{r} z} v_{\mathrm{t} z} \xi^{6}-12 A^{2} B^{2} v_{\mathrm{r} z} v_{\mathrm{t} z} \xi^{6}-4 A^{2} D^{2} v_{\mathrm{r} z} v_{\mathrm{t} z} \xi^{6}-4 B^{2} D^{2} v_{\mathrm{r} z} v_{\mathrm{t} z} \xi^{6}$ $-2 A D^{3} v_{\mathrm{t} x} v_{\mathrm{t} z} \xi^{6}-4 A^{3} D v_{\mathrm{t} x} v_{\mathrm{t} z} \xi^{6}-4 A B^{2} D v_{\mathrm{t} x} v_{\mathrm{t} z} \xi^{6}+2 A^{3} C v_{\mathrm{t} x} v_{\mathrm{t} z} \xi^{6}+2 A B^{2} C v_{\mathrm{t} x} v_{\mathrm{t} z} \xi^{6}+2 A D^{2} C v_{\mathrm{t} x} v_{\mathrm{t} z} \xi^{6}$ $-2 B D^{3} v_{\mathrm{t} y} v_{\mathrm{t} z} \xi^{6}-4 B^{3} D v_{\mathrm{t} y} v_{\mathrm{t} z} \xi^{6}-4 A^{2} B D v_{\mathrm{t} y} v_{\mathrm{t} z} \xi^{6}+2 B^{3} C v_{\mathrm{t} y} v_{\mathrm{t} z} \xi^{6}+2 B D^{2} C v_{\mathrm{t} y} v_{\mathrm{t} z} \xi^{6}+2 A^{2} B C v_{\mathrm{t} y} v_{\mathrm{t} z} \xi^{6}+B^{4} v_{\mathrm{r} x}^{2} \xi^{4}$ $+D^{4} v_{\mathrm{r} x}^{2} \xi^{4}+A^{2} B^{2} v_{\mathrm{r} x}^{2} \xi^{4}+A^{2} D^{2} v_{\mathrm{r} x}^{2} \xi^{4}+2 B^{2} D^{2} v_{\mathrm{r} x}^{2} \xi^{4}+A^{4} v_{\mathrm{r} y}^{2} \xi^{4}+D^{4} v_{\mathrm{r} y}^{2} \xi^{4}+A^{2} B^{2} v_{\mathrm{r} y}^{2} \xi^{4}+2 A^{2} D^{2} v_{\mathrm{r} y}^{2} \xi^{4}+B^{2} D^{2} v_{\mathrm{r} y}^{2} \xi^{4}$ $+A^{4} v_{\mathrm{r} z}^{2} \xi^{4}+B^{4} v_{\mathrm{r} z}^{2} \xi^{4}+2 A^{2} B^{2} v_{\mathrm{r} z}^{2} \xi^{4}+A^{2} D^{2} v_{\mathrm{r} z}^{2} \xi^{4}+B^{2} D^{2} v_{\mathrm{r} z}^{2} \xi^{4}+B^{4} v_{\mathrm{t} x}^{2} \xi^{4}+D^{4} v_{\mathrm{t} x}^{2} \xi^{4}+A^{2} B^{2} v_{\mathrm{t} x}^{2} \xi^{4}+A^{2} D^{2} v_{\mathrm{t} x}^{2} \xi^{4}$ $+2 B^{2} D^{2} v_{\mathrm{t} x}^{2} \xi^{4}+A^{4} v_{\mathrm{t} y}^{2} \xi^{4}+D^{4} v_{\mathrm{t} y}^{2} \xi^{4}+A^{2} B^{2} v_{\mathrm{t} y}^{2} \xi^{4}+2 A^{2} D^{2} v_{\mathrm{t} y}^{2} \xi^{4}+B^{2} D^{2} v_{\mathrm{t} y}^{2} \xi^{4}+A^{4} v_{\mathrm{t} z}^{2} \xi^{4}+B^{4} v_{\mathrm{t} z}^{2} \xi^{4}+2 A^{2} B^{2} v_{\mathrm{t} z}^{2} \xi^{4}$ $+A^{2} D^{2} v_{\mathrm{t} z}^{2} \xi^{4}+B^{2} D^{2} v_{\mathrm{t} z}^{2} \xi^{4}-2 A B^{3} v_{\mathrm{r} x} v_{\mathrm{r} y} \xi^{4}-2 A B D^{2} v_{\mathrm{r} x} v_{\mathrm{r} y} \xi^{4}-2 A^{3} B v_{\mathrm{r} x} v_{\mathrm{r} y} \xi^{4}-2 A D^{3} v_{\mathrm{r} x} v_{\mathrm{r} z} \xi^{4}-2 A^{3} D v_{\mathrm{r} x} v_{\mathrm{r} z} \xi^{4}$ $-2 A B^{2} D v_{\mathrm{r} x} v_{\mathrm{r} z} \xi^{4}-2 B D^{3} v_{\mathrm{r} y} v_{\mathrm{r} z} \xi^{4}-2 B^{3} D v_{\mathrm{r} y} v_{\mathrm{r} z} \xi^{4}-2 A^{2} B D v_{\mathrm{r} y} v_{\mathrm{r} z} \xi^{4}-2 B^{4} v_{\mathrm{r} x} v_{\mathrm{t} x} \xi^{4}-2 D^{4} v_{\mathrm{r} x} v_{\mathrm{t} x} \xi^{4}-2 A^{2} B^{2} v_{\mathrm{r} x} v_{\mathrm{t} x} \xi^{4}$ $-2 A^{2} D^{2} v_{\mathrm{r} x} v_{\mathrm{t} x} \xi^{4}-4 B^{2} D^{2} v_{\mathrm{r} x} v_{\mathrm{t} x} \xi^{4}+2 A B^{3} v_{\mathrm{r} y} v_{\mathrm{t} x} \xi^{4}+2 A B D^{2} v_{\mathrm{r} y} v_{\mathrm{t} x} \xi^{4}+2 A^{3} B v_{\mathrm{r} y} v_{\mathrm{t} x} \xi^{4}+2 A D^{3} v_{\mathrm{r} z} v_{\mathrm{t} x} \xi^{4}$ $+2 A^{3} D v_{\mathrm{r} z} v_{\mathrm{t} x} \xi^{4}+2 A B^{2} D v_{\mathrm{r} z} v_{\mathrm{t} x} \xi^{4}+2 A B^{3} v_{\mathrm{r} x} v_{\mathrm{t} y} \xi^{4}+2 A B D^{2} v_{\mathrm{r} x} v_{\mathrm{t} y} \xi^{4}+2 A^{3} B v_{\mathrm{r} x} v_{\mathrm{t} y} \xi^{4}-2 A^{4} v_{\mathrm{r} y} v_{\mathrm{t} y} \xi^{4}-2 D^{4} v_{\mathrm{r} y} v_{\mathrm{t} y} \xi^{4}$ 


$$
\begin{aligned}
& -2 A^{2} B^{2} v_{\mathrm{r} y} v_{\mathrm{t} y} \xi^{4}-4 A^{2} D^{2} v_{\mathrm{r} y} v_{\mathrm{t} y} \xi^{4}-2 B^{2} D^{2} v_{\mathrm{r} y} v_{\mathrm{t} y} \xi^{4}+2 B D^{3} v_{\mathrm{r} z} v_{\mathrm{t} y} \xi^{4}+2 B^{3} D v_{\mathrm{r} z} v_{\mathrm{t} y} \xi^{4}+2 A^{2} B D v_{\mathrm{r} z} v_{\mathrm{t} y} \xi^{4} \\
& -2 A B^{3} v_{\mathrm{t} x} v_{\mathrm{t} y} \xi^{4}-2 A B D^{2} v_{\mathrm{t} x} v_{\mathrm{t} y} \xi^{4}-2 A^{3} B v_{\mathrm{t} x} v_{\mathrm{t} y} \xi^{4}-2 A D^{3} v_{\mathrm{r} x} v_{\mathrm{t} z} \xi^{4}-2 A^{3} D v_{\mathrm{r} x} v_{\mathrm{t} z} \xi^{4}-2 A B^{2} D v_{\mathrm{r} x} v_{\mathrm{t} z} \xi^{4} \\
& -2 B D^{3} v_{\mathrm{r} y} v_{\mathrm{t} z} \xi^{4}-2 B^{3} D v_{\mathrm{r} y} v_{\mathrm{t} z} \xi^{4}-2 A^{2} B D v_{\mathrm{r} y} v_{\mathrm{t} z} \xi^{4}+2 A^{4} v_{\mathrm{r} z} v_{\mathrm{t} z} \xi^{4}+2 B^{4} v_{\mathrm{r} z} v_{\mathrm{t} z} \xi^{4}+4 A^{2} B^{2} v_{\mathrm{r} z} v_{\mathrm{t} z} \xi^{4}+2 A^{2} D^{2} v_{\mathrm{r} z} v_{\mathrm{t} z} \xi^{4} \\
& +2 B^{2} D^{2} v_{\mathrm{r} z} v_{\mathrm{t} z} \xi^{4}+2 A D^{3} v_{\mathrm{t} x} v_{\mathrm{t} z} \xi^{4}+2 A^{3} D v_{\mathrm{t} x} v_{\mathrm{t} z} \xi^{4}+2 A B^{2} D v_{\mathrm{t} x} v_{\mathrm{t} z} \xi^{4}+2 B D^{3} v_{\mathrm{t} y} v_{\mathrm{t} z} \xi^{4}+2 B^{3} D v_{\mathrm{t} y} v_{\mathrm{t} z} \xi^{4} \\
& +2 A^{2} B D v_{\mathrm{t} y} v_{\mathrm{t} z} \xi^{4}
\end{aligned}
$$

